\title{
CONFORMAL INVARIANCE OF PLANAR LOOP-ERASED RANDOM WALKS AND UNIFORM SPANNING TREES
}

\author{
By Gregory F. LAWler, ${ }^{1}$ Oded Schramm And Wendelin Werner \\ Cornell University, Microsoft Corporation and Université Paris-Sud
}

This paper proves that the scaling limit of a loop-erased random walk in a simply connected domain $D \varsubsetneqq \mathbb{C}$ is equal to the radial $\mathrm{SLE}_{2}$ path. In particular, the limit exists and is conformally invariant. It follows that the scaling limit of the uniform spanning tree in a Jordan domain exists and is conformally invariant. Assuming that $\partial D$ is a $C^{1}$-simple closed curve, the same method is applied to show that the scaling limit of the uniform spanning tree Peano curve, where the tree is wired along a proper arc $A \subset \partial D$, is the chordal SLE 8 path in $\bar{D}$ joining the endpoints of $A$. A by-product of this result is that $\mathrm{SLE}_{8}$ is almost surely generated by a continuous path. The results and proofs are not restricted to a particular choice of lattice.

\section{Introduction.}

1.1. Motivation from statistical physics. One of the main goals of both probability theory and statistical physics is to understand the asymptotic behavior of random systems when the number of microscopic random inputs goes to $\infty$. These random inputs can be independent, such as a sequence of independent random variables, or dependent, as in the Ising model. Often, one wishes to understand these systems via some relevant "observables" that can be of a geometric or analytic nature. In order to understand this asymptotic behavior, one can attempt to prove convergence toward a suitable continuous model. The simplest and most important example of such random continuous models is Brownian motion, which is the scaling limit of random walks. In particular, a simple random walk on any lattice in $\mathbb{R}^{d}$ converges to (a linear image of ) Brownian motion in the scaling limit.

Physicists and chemists have observed that critical systems (i.e., systems at their phase transition point) can exhibit macroscopic randomness. Hence, various quantities related to the corresponding lattice models should converge as the mesh is refined. In fact, one of the important starting points for theoretical physicists working on two-dimensional critical models is the assumption that the continuous limit is independent of the lattice and, furthermore, displays conformal invariance. This assumption has enabled them to develop and use techniques from conformal field theory to predict the exact values of certain critical exponents. Until very

Received February 2002; revised March 2003.

${ }^{1}$ Supported in part by the NSF and the Mittag-Leffler Institute.

AMS 2000 subject classifications. 82B41.

Key words and phrases. Loop-erased random walk, uniform spanning trees, stochastic Loewner evolution. 
recently, the existence of the limit, its conformal invariance and the derivation of the exponents assuming conformal invariance remained beyond mathematical justification for the basic lattice models in critical phenomena, such as percolation, the Ising model and random cluster measures. Although there are many interesting questions about higher-dimensional systems, we will limit our discussion to two dimensions where conformal invariance plays an essential role.

1.2. Recent progress. In [38], a one-parameter family of random growth processes (loosely speaking, random curves) in two dimensions was introduced. The growth process is based on Loewner's differential equation, where the driving term is time-scaled one-dimensional Brownian motion, and is therefore called stochastic Loewner evolution, or $\mathrm{SLE}_{\kappa}$. The parameter $\kappa \geq 0$ of SLE is the time scaling constant for the driving Brownian motion. It was conjectured that the scaling limit of the loop-erased random walk (LERW) is $\mathrm{SLE}_{2}$, and this conjecture was proved to be equivalent to the conformal invariance of the LERW scaling limit (see [38]). The argument given was quite general and shows that a conformally invariant random path satisfying a mild Markovian property, which will be described below, must be SLE. On this basis, it was also conjectured there that the scaling limits of the critical percolation interface and the uniform spanning tree Peano curve are the paths of $\mathrm{SLE}_{6}$ and $\mathrm{SLE}_{8}$, respectively, and it was claimed that conformal invariance is sufficient to establish these conjectures. (For additional conjectures regarding curves tending to SLE, including the interfaces in critical random cluster models-also called FK percolation modelsfor $q \in[0,4]$, see [36].)

At some values of the parameter $\kappa$, SLE has some remarkable properties. For instance, $\mathrm{SLE}_{6}$ has a locality property (see [27]) that makes it possible to relate its outer boundary to that of planar Brownian motion. This has led to the proof of conjectures concerning planar Brownian motion and simple random walks (see [27]-[29]).

In [40] and [41], Smirnov recently proved the existence and conformal invariance of the scaling limit of critical site percolation on the two-dimensional triangular lattice: he managed to prove Cardy's formula (see [5]) which is a formula for the limit of the probability of a percolation crossing between two arcs on the boundary of the domain. Combining this information with the independence properties of percolation, Smirnov then showed that the scaling limit of the percolation interface is $\mathrm{SLE}_{6}$. This has led to the rigorous determination of critical exponents for this percolation model (see [30] and [42]).

1.3. LERW and UST defined. The uniform spanning tree (UST), which can be interpreted as the $q=0$ critical random cluster model (see [15]), is a dependent model that has many remarkable features. In particular, it is very closely related to the loop-erased random walk, whose definition (see [24]) we now briefly recall. 
Consider any finite or recurrent connected graph $G$, a vertex $a$ and a set of vertices $V$. A loop-erased random walk (LERW) from $a$ to $V$ is a random simple curve joining $a$ to $V$ obtained by erasing the loops in chronological order from a simple random walk started at $a$ and stopped upon hitting $V$. In other words, if $(\Gamma(n), 0 \leq n \leq T)$ is a simple random walk on $G$ started from $a$ and stopped at its first hitting time $T$ of $V$, the loop erasure $\beta=\left(\beta_{0}, \ldots, \beta_{\ell}\right)$ is defined inductively as follows: $\beta_{0}=a$; if $\beta_{n} \in V$, then $n=\ell$; and otherwise $\beta_{n+1}=\Gamma(k)$, where $k=1+\max \left\{m \leq T: \Gamma(m)=\beta_{n}\right\}$.

A spanning tree $T$ of a connected graph $G$ is a subgraph of $G$ such that for every pair of vertices $v, u$ in $G$ there is a unique simple path (i.e., self-avoiding) in $T$ with these vertices as endpoints. A uniform spanning tree (UST) in a finite, connected graph $G$ is a sample from the uniform probability measure on spanning trees of $G$. It has been shown in [34] that the law of the self-avoiding path with endpoints $a$ and $b$ in the UST is the same as that of the LERW from $a$ to $\{b\}$. See Figure 1.

Wilson [44] established an even stronger connection between LERW and UST by giving an algorithm to generate UST's using LERW. Wilson's algorithm runs as follows. Pick an arbitrary ordering $v_{0}, v_{1}, \ldots, v_{m}$ for the vertices in $G$. Let $T_{0}=\left\{v_{0}\right\}$. Inductively, for $n=1,2, \ldots, m$, define $T_{n}$ to be the union of $T_{n-1}$ and a (conditionally independent) LERW path from $v_{n}$ to $T_{n-1}$. (If $v_{n} \in T_{n-1}$, then $T_{n}=T_{n-1}$.) Then, regardless of the chosen order of the vertices, $T_{m}$ is a UST on $G$.

Wilson's algorithm gives a natural extension of the definition of UST to infinite recurrent graphs. In fact, for transient graphs, there are two natural definitions which often coincide, but this interesting theory is somewhat removed from the topic of this paper. Many striking properties of UST and LERW have been

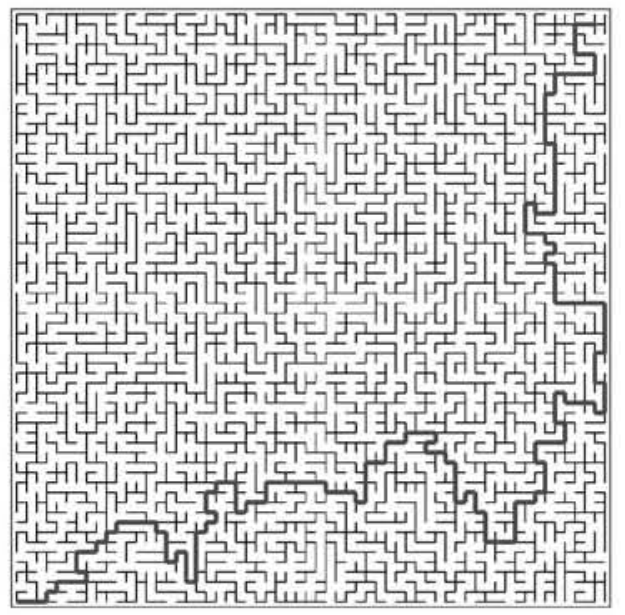

FIG. 1. The LERW in the UST. 
discovered. See [32] for a survey of UST's and [26] for a survey of properties of LERW in $\mathbb{Z}^{d}, d>2$.

Exploiting a link with domino tilings and deriving discrete analogs of CauchyRiemann equations, Kenyon (see [20] and [21]) rigorously established the values of various critical exponents predicted for the LERW (see [11], [14] and [32]) in two dimensions. In particular, he showed that the expected number of steps of a LERW joining two corners of the $N \times N$ square in the square grid $\mathbb{Z}^{2}$ is of the order of magnitude of $N^{5 / 4}$. He also showed conformal invariance for the leading term in the asymptotics of the probability that the LERW contains a given edge. This was the first mathematical evidence for full conformal invariance of the LERW scaling limit.

In [3] and [1] subsequential scaling limits of the UST measures in $\mathbb{Z}^{d}$ were shown to exist, using a compactness argument. Moreover, these papers prove that all the paths in the scaling limit that intersect a fixed bounded region are uniformly Hölder continuous. In [38] the topology of subsequential scaling limits of the UST on $\mathbb{Z}^{2}$ was determined. In particular, it was shown that every subsequential scaling limit of the LERW is a simple path.

1.4. A short description of SLE. We now briefly describe SLE; precise definitions are deferred to Section 2.1. Chordal SLE is a random growing family of compact sets $K_{t}, t \in[0, \infty)$, in the closure $\overline{\mathbb{H}}$ of the upper half plane $\mathbb{H}$. The evolution of $K_{t}$ is given by the Loewner differential equation with "driving function" Brownian motion. From [36], it is known that when $\kappa \neq 8$ the process is described by a random curve $\gamma:[0, \infty) \rightarrow \overline{\mathbb{H}}$, in the sense that, for every $t \geq 0$, $\mathbb{H} \backslash K_{t}$ is the unbounded component of $\mathbb{H} \backslash \gamma[0, t]$. A corollary of our results is that this holds for $\kappa=8$ as well. The curve $\gamma$ satisfies $\gamma(0)=0$ and $\lim _{t \rightarrow \infty} \gamma(t)=\infty$. If $\kappa \leq 4$, then $\gamma$ is a simple curve and $K_{t}=\gamma[0, t]$.

There is another version of SLE called radial SLE. Radial SLE also satisfies the description above, except that the upper half plane $\mathbb{H}$ is replaced by the unit disk $\mathbb{U}, \gamma(0)$ is on the unit circle $\partial \mathbb{U}$ and $\lim _{t \rightarrow \infty} \gamma(t)=0$.

Both radial and chordal versions of SLE may be defined in an arbitrary simply connected domain $D \varsubsetneqq \mathbb{C}$ by mapping over to $D$ using a fixed conformal map $\phi$ from $\mathbb{H}$ or $\mathbb{U}$ to $D$.

1.5. The main results of the paper. Let $D \varsubsetneqq \mathbb{C}$ be a simply connected domain with $0 \in D$. For $\delta>0$, let $\mu_{\delta}$ be the law of the loop erasure of a simple random walk on the grid $\delta \mathbb{Z}^{2}$, started at 0 and stopped when it hits $\partial D$. See Figure 2. Let $v$ be the law of the image of the radial $\mathrm{SLE}_{2}$ path under a conformal map from the unit disk $\mathbb{U}$ to $D$ fixing 0 . When the boundary of $D$ is very rough, the conformal map from $\mathbb{U}$ to $D$ might not extend continuously to the boundary, but the proof of the following theorem in fact shows that even in this case the image of the $\mathrm{SLE}_{2}$ path has a unique endpoint on $\partial D$. 


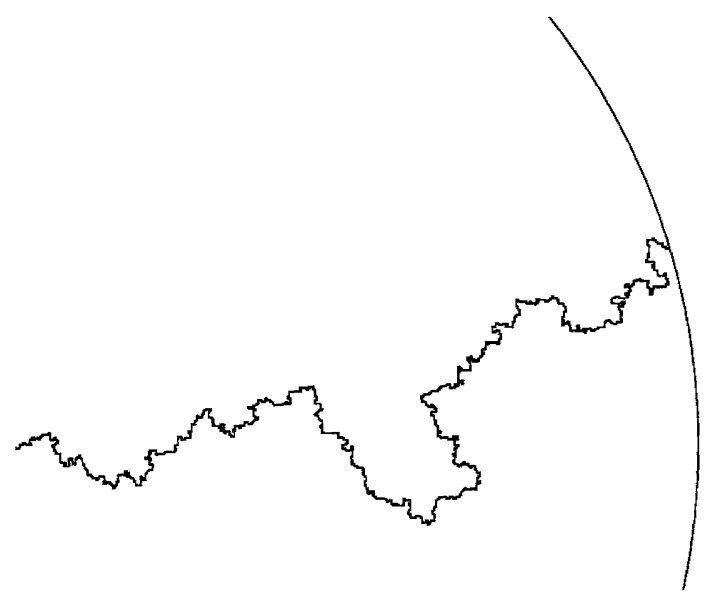

FIG. 2. A sample of the loop-erased random walk; proved to converge to radial $\mathrm{SLE}_{2}$.

On the space of unparameterized paths in $\mathbb{C}$, consider the metric $\rho(\beta, \gamma)=$ $\inf \sup _{t \in[0,1]}|\hat{\beta}(t)-\hat{\gamma}(t)|$, where the infimum is over all choices of parameterizations $\hat{\beta}$ and $\hat{\gamma}$ in $[0,1]$ of $\beta$ and $\gamma$.

THEOREM 1.1 (LERW scaling limit). The measures $\mu_{\delta}$ converge weakly to $v$ as $\delta \rightarrow 0$ with respect to the metric $\rho$ on the space of curves.

Since SLE is conformally invariant by definition, this theorem implies conformal invariance of the LERW. The theorem and proof apply also to some other walks on lattices in the plane where the scaling limit of the walk is isotropic Brownian motion. It even applies in the nonreversible setting. See Section 6 for further details.

There are two distinct definitions for the UST corresponding to a domain $D \varsubsetneqq \mathbb{C}$, as follows. Let $G_{F}(D)$ denote the subgraph of $\mathbb{Z}^{2}$ consisting of all the edges and vertices which are contained in $D$. If $G_{F}(D)$ is connected, then we refer to the UST on $G_{F}(D)$ as the UST on $D$ with free boundary conditions. Let $G_{W}(D)$ denote the graph obtained from $\mathbb{Z}^{2}$ by contracting all the vertices outside of $D$ to a single vertex (and removing edges which become loops). Then the UST on $G_{W}(D)$ is the UST on $D$ with wired boundary conditions.

Since the UST is built from the LERW via Wilson's algorithm, it is not surprising that conformal invariance of the UST scaling limit should follow from that of the LERW scaling limit. In fact, [38], Theorem 11.3, says just that.

COROLLARY 1.2 (UST scaling limit). The wired and free UST scaling limits (as defined in [38]) in a simply connected domain $D \subset \mathbb{C}$ whose boundary is a $C^{1}$-smooth simple closed curve exist and are conformally invariant. 
One can easily show, using [38], Theorem 11.1(i), that the wired tree depends continuously on the domain, and hence for that case $D$ may be an arbitrary simply connected domain. However, some regularity assumption is needed for the free UST scaling limit: conformal invariance fails for the domain whose boundary contains the topologist's sine curve (the closure of $\{x+i \sin (1 / x): x \in(0,1]\})$.

The UST Peano curve is an entirely different curve derived from the UST in two dimensions. The curve is rather remarkable, as it is a natural random path visiting every vertex in an appropriate graph or lattice. We now roughly describe two natural definitions of this curve; further details appear in Section 4.

Let $G$ be a finite planar graph, with a particular embedding in the plane, and let $G^{\dagger}$ denote its planar dual, again with a particular embedding. Then there is a bijection $e \leftrightarrow e^{\dagger}$ between the edges of $G$ and those of $G^{\dagger}$ such that, for every edge $e$ in $G, e \cap e^{\dagger}$ is a single point, and $e$ does not intersect any other edge of $G^{\dagger}$. Given a spanning tree $T$ of $G$, let $T^{\dagger}$ denote the graph whose vertices are the vertices of $G^{\dagger}$ and whose edges are those edges $e^{\dagger}$ such that $e \notin T$. It is then easy to verify that $T^{\dagger}$ is a spanning tree for $G^{\dagger}$. Therefore, if $T$ is a UST on $G$, then $T^{\dagger}$ is a UST on $G^{\dagger}$.

The UST Peano curve is a curve that winds between $T$ and $T^{\dagger}$ and separates them. More precisely, consider the graph $\hat{G}$ drawn in the plane by taking the union of $G$ and $G^{\dagger}$, where each edge $e$ or $e^{\dagger}$ is subdivided into two edges by introducing a vertex at $e \cap e^{\dagger}$. The subgraph of the planar dual $\hat{G}^{\dagger}$ of $\hat{G}$ containing all edges which do not intersect $T \cup T^{\dagger}$ is a simple closed path-the UST Peano path. See Figure 3.

Some properties of the UST Peano path on $\mathbb{Z}^{2}$ have been studied in the physics literature; see, for example, [10] and [18]. There, it has been called the Hamiltonian path on the Manhattan lattice. The reason for this name is as follows. On $\mathbb{Z}^{2}$, say, orient each horizontal edge whose $y$-coordinate is even to the right and each horizontal edge whose $y$-coordinate is odd to the left. Similarly, orient down each vertical edge whose $x$-coordinate is even and orient up each vertical edge whose
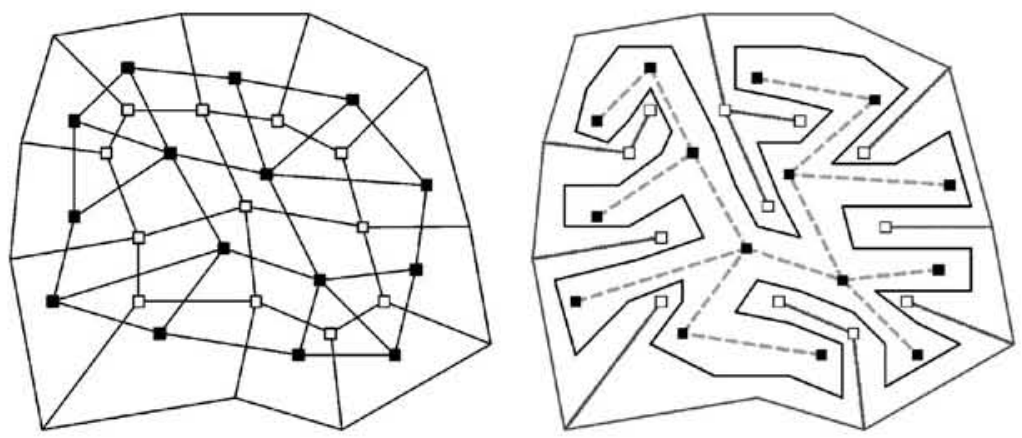

FIG. 3. The graph, dual graph, tree, dual tree and Peano curve. The vertex of the dual graph corresponding to the unbounded face is drawn as a cycle. 
$x$-coordinate is odd. Now rescale the resulting oriented graph by $1 / 2$ and translate it by $(1 / 4,1 / 4)$. It is easy to check that a Hamiltonian path (a path visiting every vertex exactly once) respecting the orientation on the resulting oriented graph is the same as the UST Peano path of $\mathbb{Z}^{2}$. It should be expected that the uniform measure on Hamiltonian paths in $\mathbb{Z}^{2}$ has the same scaling limit as that of the UST Peano path.

Given a domain $D$, one can consider the UST Peano curve for the wired or for the free UST (which is essentially the same as the wired, by duality). However, the conjecture from [38] regarding the convergence to chordal SLE pertains to the UST Peano curve associated with the tree with mixed wired and free conditions.

Let $D \subset \mathbb{C}$ be a domain whose boundary is a $C^{1}$-smooth simple closed curve and let $a, b \in \partial D$ be distinct boundary points. Let $\alpha$ and $\beta$ denote the two complementary arcs of $\partial D$ whose endpoints are $a$ and $b$. For all $\delta>0$, consider an approximation $G_{\delta}$ of the domain $D$ in the grid $\delta \mathbb{Z}^{2}$. (A precise statement of what it means for $G_{\delta}$ to be an approximation of $D$ will be given in Section 4.) Let $\gamma_{\delta}$ denote the Peano curve associated to the UST on $G_{\delta}$ with wired boundary near $\alpha$ and free boundary near $\beta$. Then $\gamma_{\delta}$ may be considered as a path in $D$ from a point near $a$ to a point near $b$.

THEOREM 1.3 (UST Peano path scaling limit). The UST Peano curve scaling limit in $D$ with wired boundary on $\alpha$ and free boundary on $\beta$ exists and is equal to the image of the chordal $\mathrm{SLE}_{8}$ path under any conformal map from $\mathbb{H}$ to $D$ mapping 0 to $a$ and $\infty$ to $b$.

Again, the convergence is weak convergence of measures with respect to the metric $\rho$. Figure 4 shows a sample of the UST Peano path on a fine grid.

As explained above, it was proved in [36] that each $\mathrm{SLE}_{\kappa}$ is generated by a path, except for $\kappa=8$. In Section 4.4, the remaining case $\kappa=8$ is proved, using the convergence of the Peano curve.

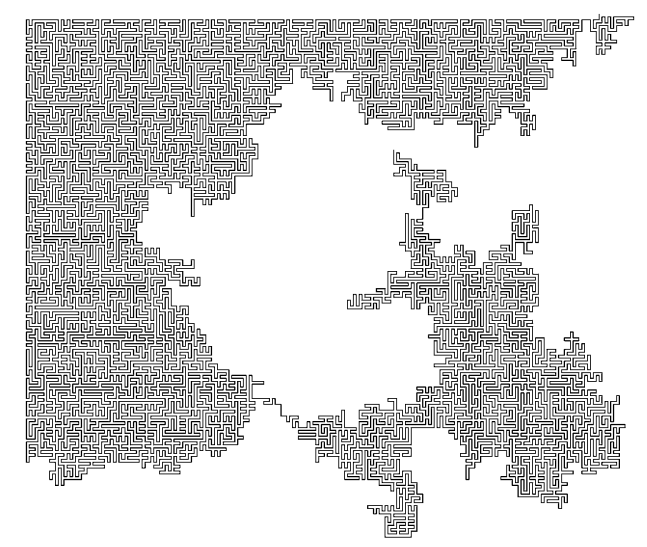

FIG. 4. An arc from a sample of the UST Peano path; proved to converge to chordal SLE 8 . 
Corollary 1.2 and Theorem 1.3 (and their proofs) apply to other reversible walks on planar lattices (the self-duality of $\mathbb{Z}^{2}$ does not play an important role); see Section 6.

To add perspective, we note that the convergence to SLE of the LERW and the UST Peano curve are two boundary cases of the conjectured convergence in [36] of the critical FK random cluster measures with parameter $q \in(0,4)$. For these parameter values, the scaling limit of the interface of a critical cluster with mixed boundary values is conjectured to converge to chordal $\operatorname{SLE}_{\kappa}(q)$, where $\kappa(q)=4 \pi / \cos ^{-1}(-\sqrt{q} / 2)$. The boundary case $\kappa(0)=8$ corresponds to the convergence of the UST Peano path to $\mathrm{SLE}_{8}$.

The outer boundary of the scaling limit of a macroscopic critical cluster is not the same as the scaling limit of a critical cluster outer boundary, because of "fjords" which are pinched off in the limit. The former is conjectured to "look like" $\mathrm{SLE}_{16 / \kappa(q)}$, but a precise form of this conjecture is not yet known. In the case $q=0$, however, such a correspondence is easy to explain. In $\mathbb{Z}^{2}$, an arc of the Peano curve is surrounded on one side by a simple path in the tree, and on the other side by a simple path in the dual tree. Both these paths are LERWs. Similar correspondences exist for the UST in a subdomain of $\mathbb{R}^{2}$, but one has to set appropriate boundary conditions. Thus, the convergence of LERW to $\mathrm{SLE}_{2}$ also corresponds to the case $q=0$, as $16 / \kappa(0)=2$.

Suppose that $0 \in D$ and $\alpha, \beta \subset \partial D$, as before. Consider the simple random walk on $\delta \mathbb{Z}^{2}$ which is reflected off $\beta$ and stopped when it hits $\alpha$. Using an analogous method to that of the present paper, one could handle the scaling limit of the loop erasure of this walk. It is described by a variant of $\mathrm{SLE}_{2}$ where the driving term is Brownian motion with time scaled by 2 , but having an additional drift. The drift is not constant, but can be explicitly computed.

The identification of the scaling limit as one of the SLEs should facilitate the derivation of critical exponents and also the asymptotic probabilities of various events, including some results which have not been predicted by arguments from physics. This was the case for critical site percolation on the triangular grid; see [30], [39], [40] and [42].

1.6. Some comments about the proof. Since a loop-erased random walk is obtained in a deterministic way from a simple random walk (by erasing its loops) and since a simple random walk converges to Brownian motion in the scaling limit, it is natural to think that the scaling limit of the LERW should simply be the process obtained by erasing the loops from a planar Brownian motion. The problem with this approach is that planar Brownian motion has loops at every scale, so that there is no simple algorithm to erase loops. In particular, there is no "first" loop. Our proof does use the relation between the LERW and simple random walks, combined with the fact that quantities related to simple random walks, such as hitting probabilities, converge to their continuous conformally invariant counterparts. 
The proof of each of our main theorems is naturally divided into two parts. The first part establishes the convergence to SLE with respect to a weaker topology than the topology induced by the metric $\rho$ of paths; namely, we show that the Loewner driving process for the discrete random path converges to a Brownian motion. This part of the proof, which we consider to be the more important one, is essentially self-contained. The second part uses some regularity properties of the discrete processes from [38] to prove convergence with respect to the stronger topology.

The method for the first part can be considered as a rather general method for identifying the scaling limit of a dependent system that is conjectured to be conformally invariant. It requires having some "observable" quantity that can be estimated well and a mild Markovian property, which we now describe. Suppose that to every simply connected domain $D$ containing 0 there is associated a random path $\gamma$ from $\partial D$ to 0 (e.g., the orientation reversal of the LERW). The required property is that if $\beta$ is an arc with one endpoint in $\partial D$ and we condition on $\beta \subset \gamma$ (assuming this has positive probability, say), then the conditioned distribution of $\gamma \backslash \beta$ is the same as the random path in the domain $D \backslash \beta$ conditioned to start at the other endpoint $q$ of $\beta$. [Thus, $(D \backslash \beta, q)$ is the state of a Markov chain whose transitions correspond to adding edges from $\gamma$ to $\beta$ and modifying $q$ appropriately.] Interestingly, among the discrete processes conjectured to converge to SLE, the LERW is the only one where the verification of this property is not completely trivial. [For the LERW it is not trivial, but not difficult; see part (iii) of Lemma 3.2.] The statement of this property for the UST Peano curve is given in Lemma 4.1. The fact that SLE satisfies this property follows from the Markovian property of its driving Brownian motion.

The particular choice of observable is not so important. What is essential is that one can conveniently calculate the asymptotics of the observable for appropriate large-scale configurations. The particular observable that we have chosen for the LERW convergence is the expected number of visits to a vertex $v$ by the simple random walk generating the LERW. Conformal invariance is not assumed but comes out of the calculation-hitting probabilities for random walks are discrete harmonic functions, which converge to continuous harmonic functions. One technical issue is to establish this convergence without any boundary smoothness assumption. Once the observable has been approximated, the conditional expectation and variance of increments of the Loewner driving function for the discrete process can be estimated, and standard techniques (the Skorohod embedding) can be used to show that this random function approaches the appropriate Brownian motion.

Although Theorem 1.3 can probably be derived with some work from Corollary 1.2, instead, to illustrate our method, we prove it by applying again the same general strategy of the proof of Theorem 1.1, with the choice of a different observable. 
Actually, it is easier to explain the main ideas behind the proof of Theorem 1.3. Fix some vertex $v$ in $D$ and a subarc $\alpha_{1} \subset \alpha$. Let $\mathcal{A}$ be the event that the UST path (not the Peano path, but the path contained in the UST) from $v$ to $\alpha$ hits $\alpha_{1}$. By Wilson's algorithm the probability of $\mathcal{A}$ is the same as the probability that a simple random walk started at $v$ reflected off $\beta$ first hits $\alpha$ in $\alpha_{1}$. The latter probability can be estimated directly. If $\gamma[0, n]$ denotes the restriction of the Peano path to its first $n$ steps, then $\mathbf{P}[\mathcal{A} \mid \gamma[0, n]]$, the probability of $\mathcal{A}$ conditioned on $\gamma[0, n]$, is clearly a martingale with respect to $n$. But, by the Markovian property discussed above, the value of $\mathbf{P}[\mathcal{A} \mid \gamma[0, n]]$ may be estimated in precisely the same way that $\mathbf{P}[\mathcal{A}]$ is estimated. The estimate turns out to be a function of the conformal geometry of the configuration $\left(v, D \backslash \gamma[0, n], \gamma(n), \alpha_{1}, \beta\right)$. Knowing that this is a martingale for two appropriately chosen vertices $v$ is sufficient to characterize the large-scale behavior of $\gamma$.

As mentioned above, in the case of the LERW, the observable we chose to look at is the expected number of visits to a fixed vertex $v$ by the simple random walk $\Gamma$ generating the LERW $\gamma$. The walk $\Gamma$ can be considered as the union of $\gamma$ with a sequence of loops $\Gamma^{j}$ based at vertices of $\gamma$. We look at the conditioned expectation of the number of visits of $\Gamma$ to $v$ given an arc $\tilde{\gamma}$ of $\gamma$ adjacent to the boundary of the domain. This is clearly a martingale with respect to the filtration obtained by taking larger and larger arcs $\tilde{\gamma} \subset \gamma$. This quantity falls into two parts: the visits to $v$ in the loops $\Gamma^{j}$ based at $\tilde{\gamma}$, and those that are not. Each of these two parts can be well estimated by random walk calculations. Translating the fact that this is a martingale to information about the Loewner driving process for $\gamma$ inevitably leads to the identification of this driving process as appropriately scaled Brownian motion.

Actually, we first had a longer proof of convergence of the LERW to the $\mathrm{SLE}_{2}$, based on the fact that it is possible to construct the hull of a Brownian motion by adding Brownian loops to $\mathrm{SLE}_{2}$. This can be viewed as a particular case of the restriction properties of $\mathrm{SLE}_{\kappa}$ with Brownian loops added, which we study in [31]. Let us also mention the following related open question. Consider a sequence of simple random walks $S^{k}(n)$ on a lattice with lattice spacing $\delta_{k} \rightarrow 0$, from $S^{k}(0)=0$ to $\partial \mathbb{U}$, and let $\gamma^{k}$ denote the corresponding loop-erased paths. Theorem 1.1 shows that one can find a subsequence such that the law of the pair $\left(\gamma^{k}, S^{k}\right)$ converges to a coupling of $\mathrm{SLE}_{2}$ with Brownian motion [i.e., a law for a pair $(X, Y)$, where $X$ has the same distribution as the $\mathrm{SLE}_{2}$ path and $Y$ has the same distribution as Brownian motion]. The question is whether, in this coupling, $\mathrm{SLE}_{2}$ is a deterministic function of the Brownian motion. In other words, is it possible to show that this is not a deterministic procedure to erase loops from a Brownian motion?

2. Preliminaries. The reading of this paper requires some background knowledge in several different fields. Some background about Loewner's equation and SLE is reviewed in the next section. It is assumed that the reader is familiar 
with some of the basic properties of Brownian motion (definition, strong Markov property, etc.). Some of the basic properties of conformal maps (Riemann's mapping theorem, compactness, Koebe distortion) are also needed for the proof. This material may be learned from the first two chapters of [35], for example. In terms of the theory of conformal mappings, this suffices for understanding the argument showing that the driving process of the LERW converges to Brownian motion. For improving the topology of convergence, some familiarity with the notion of extremal length (a.k.a. extremal distance) is also required. A possible source for that is [2]. The reader also needs to know some of the very basic properties of harmonic measure.

2.1. Loewner's equation and SLE. We now review some facts concerning Loewner's equations and stochastic Loewner evolutions. For more details, see, for example, [27], [28], [36] and [38].

Suppose that $D \varsubsetneqq \mathbb{C}$ is a simply connected domain with $0 \in D$. Then there is a unique conformal homeomorphism $\psi=\psi_{D}: D \rightarrow \mathbb{U}$ which is onto the unit disk $\mathbb{U}=\{z \in \mathbb{C}:|z|<1\}$ such that $\psi_{D}(0)=0$ and $\psi_{D}^{\prime}(0)$ is a positive real. If $D \subset \mathbb{U}$, then $\psi_{D}^{\prime}(0) \geq 1$, and $\log \psi_{D}^{\prime}(0)$ is called the capacity of $\overline{\mathbb{U}} \backslash D$ from 0 .

Now suppose that $\eta:[0, \infty] \rightarrow \overline{\mathbb{U}}$ is a continuous simple curve in the unit disk with $\eta(0) \in \partial \mathbb{U}, \eta(\infty)=0$ and $\eta(0, \infty] \subset \mathbb{U}$. For each $t \geq 0$, set $K_{t}:=\eta[0, t]$, $U_{t}:=\mathbb{U} \backslash K_{t}$ and $g_{t}:=\psi_{U_{t}}$. Since $t \mapsto g_{t}^{\prime}(0)$ is increasing (by the Schwarz lemma, say), one can reparameterize the path in such a way that $g_{t}^{\prime}(0)=\exp (t)$. If that is the case, we say that $\eta$ is parameterized by capacity from 0 . By standard properties of conformal maps (see [35], Proposition 2.5), for each $t \in[0, \infty)$ the limit

$$
W(t):=\lim _{z \rightarrow \eta(t)} g_{t}(z)
$$

where $z$ tends to $\eta(t)$ from within $\mathbb{U} \backslash \eta[0, t]$, exists. One can also verify that

$$
W:[0, \infty) \rightarrow \partial \mathbb{U}
$$

is continuous. Assuming the parameterization by capacity, Loewner's theorem states that $g_{t}$ satisfies the differential equation

$$
\partial_{t} g_{t}(z)=-g_{t}(z) \frac{g_{t}(z)+W(t)}{g_{t}(z)-W(t)} .
$$

It is also clear that

$$
\forall z \in \mathbb{U}, \quad g_{0}(z)=z .
$$

We call $(W(t), t \geq 0)$ the driving function of the curve $\eta$.

The driving function $W$ is sufficient to recover the two-dimensional path $\eta$, because the procedure may be reversed, as follows. Suppose that $W:[0, \infty) \rightarrow \partial \mathbb{U}$ is continuous. Then for every $z \in \overline{\mathbb{U}}$ there is a solution $g_{t}(z)$ of the ODE (2.1) with initial value $g_{0}(z)=z$ up to some time $\tau(z) \in(0, \infty]$, beyond which the solution 
does not exist. In fact, if $\tau(z)<\infty$ and $z \neq W(0)$, then we have $\lim _{t \uparrow \tau(z)} g_{t}(z)-$ $W(t)=0$, since this is the only possible reason the ODE cannot be solved beyond time $\tau(z)$. Then one defines $K_{t}:=\{z \in \overline{\mathbb{U}}: \tau(z) \leq t\}$ and $D_{t}:=\mathbb{U} \backslash K_{t}$ is the domain of definition of $g_{t}$. The set $K_{t}$ is called the hull at time $t$. If $W$ arises from a simple path $\eta$ as described in the previous section, then we can recover $\eta$ from $W$ by using $\eta(t)=g_{t}^{-1}(W(t))$. However, if $W:[0, \infty) \rightarrow \mathbb{U}$ is an arbitrary continuous driving function, then, in general, $K_{t}$ need not be a path, and even if it is a path, it does not have to be a simple path.

Radial $\mathrm{SLE}_{\kappa}$ is the process $\left(K_{t}, t \geq 0\right)$, where the driving function $W(t)$ is set to be $W(t):=\exp \left(i B_{\kappa t}\right)$, where $B:[0, \infty) \rightarrow \mathbb{R}$ is Brownian motion. Often, one takes the starting point $B_{0}$ to be random uniform in $[0,2 \pi]$. It has been shown in [36] that the hull $K_{t}$ is a.s. a simple curve for every $t>0$ if $\kappa \leq 4$ and that, a.s. for every $t>0, K_{t}$ is not a simple curve if $\kappa>4$. For every $\kappa \geq 0$, there is a.s. some random continuous path $\eta:[0, \infty) \rightarrow \overline{\mathbb{U}}$ such that, for all $t>0, D_{t}$ is the component of $\mathbb{U} \backslash \eta[0, t]$ containing 0 . When $\kappa \neq 8$, this was proved in [36], while for $\kappa=8$ this will be proven in the current paper. This path is called the radial SLE path.

Suppose that $D$ is a simply connected domain containing 0 . If $\gamma$ is a continuous simple curve joining $\partial D$ to 0 with only an endpoint in $\partial D$, one can reparameterize the path $\eta:=\psi \circ \gamma$ according to capacity and find its driving function $W$, as before. The conformal map

$$
\hat{g}_{t}=\psi_{D \backslash \gamma[0, t]}: D \backslash \gamma[0, t] \rightarrow \mathbb{U}
$$

still satisfies (2.1), but this time, $\hat{g}_{0}=\psi_{D}$. (Here, the parameterization chosen for $\gamma$ is according to the capacity of $\psi \circ \gamma[0, t]$.) Radial SLE in $D$ is then simply the image under $\psi_{D}^{-1}$ of radial SLE in the unit disk.

Similarly, one can encode continuous simple curves $\eta$ from 0 to $\infty$ in the closed upper half plane $\overline{\mathbb{H}}$ via a variant of Loewner's equation. For each time $t \geq 0$, there is a unique conformal map $g_{t}$ from $H_{t}:=\mathbb{H} \backslash \eta[0, t]$ onto $\mathbb{H}$ satisfying the so-called hydrodynamic normalization

$$
\lim _{z \rightarrow \infty} g_{t}(z)-z=0,
$$

where $z \rightarrow \infty$ in $\mathbb{H}$. If we write $g_{t}(z)=z+a(t) z^{-1}+o\left(z^{-1}\right)$ near $\infty$, it turns out that $a(t)$ is monotone. Consequently, one can reparameterize $\eta$ in such a way that $a(t)=2 t$, that is, $g_{t}(z)=z+2 t z^{-1}+o\left(z^{-1}\right)$ when $z \rightarrow \infty$. This parameterization of $\eta$ is called the parameterization by capacity from $\infty$. (This notion of capacity is analogous to the notion of capacity in the radial setting; however, these are two distinct notions and should not be confused.) If $g: \mathbb{H} \backslash K \rightarrow \mathbb{H}$ is the conformal homeomorphism satisfying the hydrodynamic normalization, then $\lim _{z \rightarrow \infty}\left(g_{t}(z)-z\right) z / 2$ is called the capacity of $K$ from $\infty$. Assuming that $\eta$ is parameterized by capacity, the following analog of Loewner's 
equation holds:

$$
\forall t>0, \forall z \in H_{t}, \quad \partial_{t} g_{t}(z)=\frac{2}{g_{t}(z)-W(t)},
$$

where the driving function $W$ is again defined by $W(t):=g_{t}(\eta(t))$. As above, $\eta$ is determined by $W$.

Conversely, suppose that $W$ is a real-valued continuous function. For $z \in \overline{\mathbb{H}}$, one can solve the differential equation (2.4) starting with $g_{0}(z)=z$, up to the first time $\tau(z)$ where $g_{t}(z)$ and $W(z)$ collide [possibly, $\tau(z)=\infty$ ]. Let the hull be defined by $K_{t}:=\{z \in \overline{\mathbb{H}}: \tau(z) \leq t\}$. Then $g_{t}: \mathbb{H} \backslash K_{t} \rightarrow \mathbb{H}$ is a conformal map onto $\mathbb{H}$, and $g_{0}(z)=z$. In general, $K_{t}$ is not necessarily a simple curve. If $W(t)=B_{\kappa t}$, then $\left(K_{t}, t \geq 0\right)$ is called chordal $\mathrm{SLE}_{\kappa}$.

It turns out (see [28], Section 4.1) that the local properties of chordal $\mathrm{SLE}_{\kappa}$ and of radial $\mathrm{SLE}_{\kappa}$ are essentially the same. [That is the reason the normalization $a(t)=2 t$ was chosen over the seemingly more natural $a(t)=t$.] In particular, for every $\kappa$, chordal $\mathrm{SLE}_{\kappa}$ is generated by a random continuous path, called the chordal $\mathrm{SLE}_{\kappa}$ path.

At some points in our proofs, we will need the following simple observation.

LEMMA 2.1 (Diameter bounds on $K_{t}$ ). There is a constant $C>0$ such that the following always holds. Let $W:[0, \infty) \rightarrow \mathbb{R}$ be continuous and let $\left(K_{t}, t \geq 0\right)$ be the corresponding hull for Loewner's chordal equation (2.4) with driving function W. Set

$$
k(t):=\sqrt{t}+\max \{|W(s)-W(0)|: s \in[0, t]\} .
$$

Then

$$
\forall t \geq 0, \quad C^{-1} k(t) \leq \operatorname{diam} K_{t} \leq C k(t) .
$$

Similarly, when $K_{t} \subset \overline{\mathbb{U}}$ is the radial hull for a continuous driving function $W:[0, \infty) \rightarrow \partial \mathbb{U}$, then

$$
\forall t \geq 0, \quad C^{-1} \min \{k(t), 1\} \leq \operatorname{diam} K_{t} \leq C k(t) .
$$

PROOF. This lemma can be derived by various means. We will only give a detailed argument in the radial case. The chordal case is actually easier and can be derived using the same methods. It can also be seen as a consequence of the result in the radial setting (because chordal Loewner equations can be interpreted as scaling limits of radial Loewner equations).

We start by proving the upper bound on $\operatorname{diam} K_{t}$. Let $\delta \geq \max \{\mid W(s)-$ $W(0) \mid: s \in[0, t]\}$. Then, as long as $\left|g_{t}(z)-W(0)\right| \geq 3 \delta$, we have $\left|\partial_{t} g_{t}(z)\right| \leq 1 / \delta$. Hence, if $|z-W(0)| \geq 4 \delta$, then, for all $t \leq \delta^{2},\left|g_{t}(z)-z\right| \leq \delta$ and therefore $z \notin K_{t}$. Hence, $\operatorname{diam} K_{t} \leq 8 k(t)$. 
In order to derive the lower bound, we will compare capacity with harmonic measure. It is sufficient to consider the case where diam $K_{t}<1 / 10$. Let $\mu$ denote the harmonic measure on $K_{t} \cup \partial \mathbb{U}$ from 0 . Because $K_{t}$ is contained in the disk of radius diam $K_{t}$ with center $W(0) \in K_{t} \cap \partial \mathbb{U}$, there is a universal constant $c$ such that $\mu\left(K_{t}\right) \leq c \operatorname{diam} K_{t}$. Hence, it suffices to give a lower bound for $\mu\left(K_{t}\right)$.

Since $g_{t}(z) / z$ is analytic and nonzero in a neighborhood of 0 , the function $h(z)=\log \left|g_{t}(z)\right|-\log |z|$ is harmonic in $U_{t}:=\mathbb{U} \backslash K_{t}$. Note that $h(0)=t$. Because $\left|g_{t}(z)\right| \rightarrow 1$ as $z$ tends to the boundary of $U_{t}$, the mean value property of $h \circ g_{t}^{-1}$ implies the following relation between harmonic measure and capacity: $t=h(0)=\int \log (1 /|z|) d \mu(z)$. Since $K_{t}$ contains points in $\partial \mathbb{U}$ and $\operatorname{diam} K_{t} \leq 1 / 10$, we have $\log (1 /|z|) \leq c^{\prime} \operatorname{diam} K_{t}$ for all $z \in K_{t}$. Therefore, $t \leq c^{\prime} \mu\left(K_{t}\right) \operatorname{diam} K_{t} \leq c^{\prime \prime}\left(\operatorname{diam} K_{t}\right)^{2}$.

It now remains to compare $\mu\left(K_{t}\right)$ and $|W(t)-W(0)|$. We still assume that diam $K_{t}<1 / 10$. Let $A_{t}:=\partial \mathbb{U} \backslash g_{t}\left(\overline{\mathbb{U}} \backslash K_{t}\right)$. If $z \in \partial \mathbb{U} \backslash K_{s}$ and $s \leq t$, then (2.1) shows that $\partial_{u}\left|g_{s+u}(z)-W(s)\right| \geq 0$ at $u=0$. This implies that $\left(A_{s}, s \leq t\right)$ is nondecreasing. Hence, for all $s \leq t$, we have $W(0) \in A_{0} \subset A_{t}$ and $W(s) \in A_{s} \subset A_{t}$ so that $|W(s)-W(0)|$ is bounded by the length of $A_{t}$, which is equal to $2 \pi \mu\left(K_{t}\right)$. This completes the proof of the lemma.

2.2. A discrete harmonic measure estimate. In this section, we introduce some notation and state an estimate relating discrete harmonic measure and continuous harmonic measure in domains in the plane. In order to get more quickly to the core of our method in Section 3.2, we postpone the proof of the harmonic measure estimate to Section 5.

A grid domain $D$ is a domain whose boundary consists of edges of the grid $\mathbb{Z}^{2}$. For an arbitrary domain $D \subset \mathbb{C}$, and $p \in D$ define the inner radius of $D$ with respect to $p$,

$$
\operatorname{rad}_{p}(D):=\inf \{|z-p|: z \notin D\} .
$$

Let $\mathfrak{D}$ denote the set of all simply connected grid domains such that $0<\operatorname{rad}_{0}(D)<\infty$ (i.e., $D \neq \mathbb{C}$ and $0 \in D$ ).

Points in $\mathbb{R}^{2}=\mathbb{C}$ with integer coordinates will be called vertices, or lattice points. Let $V(D):=D \cap \mathbb{Z}^{2}$ denote the lattice points in $D$.

Let $D \in \mathfrak{D}$ and let $v$ be a vertex in $\partial D$. If $\partial D$ contains more than one edge incident with $v$, then it may happen that the intersection of $D$ with a small disk centered at $v$ will not be connected. Hence, as viewed from $D, v$ appears as more than one vertex. In particular, $\psi=\psi_{D}$ does not extend continuously to $v$. This is a standard issue in conformal mapping theory, which is often resolved by introducing the notion of prime ends. But in the present case, there is a simpler solution which suffices for our purposes. Suppose $v \in \mathbb{Z}^{2} \cap \partial D$ and $e$ is an edge incident with $v$ that intersects $D$. The set of such pairs $w=(v, e)$ will be denoted $V_{\partial}(D)$. If $\psi: D \rightarrow \mathbb{U}$ is conformal, then $\psi(w)$ will be shorthand for the 
limit of $\psi(z)$ as $z \rightarrow v$ along $e$ (which always exists, by [35], Proposition 2.14). Similarly, if a random walk first exits $D$ at $v$, we say that it exited $D$ at $w$ if the edge $e$ was used when first hitting $v$. A reader of this paper who chooses to be sloppy and not distinguish between $v$ and $w$ will not lose anything in the way of substance. We will not always be so careful to make this distinction.

If $a \in V(D)$ and $b \in V(D) \cup V_{\partial}(D)$, define $H(a, b)=H_{D}(a, b)$ as the probability that the simple random walk started from $a$ and stopped at its first exit time of $D$ visits $b$.

For any $w \in D$ and $u \in V_{\partial}(D)$, we define

$$
\lambda=\lambda(w, u ; D):=\frac{1-|\psi(w)|^{2}}{|\psi(w)-\psi(u)|^{2}}=\operatorname{Re}\left(\frac{\psi(u)+\psi(w)}{\psi(u)-\psi(w)}\right) .
$$

Note that $\lambda$ is also equal to the imaginary part of the image of $w$ by the conformal map from $D$ onto the upper half plane that maps 0 onto $i$ and $u$ to $\infty$. It is also the limit when $\epsilon \rightarrow 0$ of the ratio between the harmonic measure in $D$ of the $\epsilon$ neighborhood of $u$ in $\partial D$, taken, respectively, at $w$ and at 0 (i.e., it corresponds to the Poisson kernel). Therefore, $\lambda$ can be viewed as the continuous analog of $H(w, u) / H(0, u)$. Note that the function $h(w)=H(w, u) / H(0, u)$ is discrete harmonic on $V(D)$, which means that $h(w)$ is equal to the average of $h$ on the neighbors of $w$ when $w \in V(D)$.

PROPOSITION 2.2 (Hitting probability). For every $\epsilon>0$ there is some $r_{0}>0$ such that the following holds. Let $D \in \mathfrak{D}$ satisfy $\operatorname{rad}_{0}(D)>r_{0}$ and let $u \in V_{\partial}(D)$ and $w \in V(D)$. Suppose $\left|\psi_{D}(w)\right| \leq 1-\epsilon$ and $H(0, u) \neq 0$. Then

$$
\left|\frac{H(w, u)}{H(0, u)}-\lambda(w, u ; D)\right|<\epsilon .
$$

The proof is given in Section 5 .

\section{Conformal invariance of the LERW.}

3.1. Loop-erased random walk background. We now recall some well-known facts concerning loop-erased random walks.

LEMMA 3.1 (LERW reversal). Let $D \in \mathfrak{D}$ and let $\Gamma$ be a simple random walk from 0 stopped when it hits $\partial D$. Let $\beta$ be the loop erasure of $\Gamma$ and let $\gamma$ be the loop erasure of the time reversal of $\Gamma$. Then $\gamma$ has the same distribution as the time reversal of $\beta$.

See [25]. A simpler proof follows immediately from the symmetry of (12.2.3) in [26]. This result (and the proofs) also holds if we condition $\Gamma$ to exit $\partial D$ at a prescribed $u \in V_{\partial}(D)$, which corresponds to the event $\{\gamma \cap \partial D=\{u\}\}=$ $\{\beta \cap \partial D=\{u\}\}$ (assuming this has positive probability). 
Throughout our proof, we will use the simple random walk $\Gamma$ and the loop erasure $\gamma=\left(\gamma_{0}, \gamma_{1}, \ldots, \gamma_{\ell}\right)$ of its time reversal (so that $\gamma_{0} \in \partial D$ and $\gamma_{\ell}=0$ ). We use $D_{j}$ to denote the grid domains $D_{j}:=D \backslash \bigcup_{i=0}^{j-1}\left[\gamma_{i}, \gamma_{i+1}\right]$. Define, for $j \in\{0,1, \ldots, \ell\}$,

$$
n_{j}:=\min \left\{n \geq 0: \Gamma(n)=\gamma_{j}\right\}
$$

and note that $n_{j+1}<n_{j}$ for $j=0,1, \ldots, \ell-1$ by the definition of $\gamma$. Also set

$$
\Gamma^{j+1}:=\Gamma\left[n_{j+1}, n_{j}\right] .
$$

More precisely, consider $\Gamma^{j}$ as the grid path given by

$$
\Gamma^{j}(m):=\Gamma\left(m+n_{j}\right), \quad m=0,1, \ldots, n_{j-1}-n_{j} .
$$

LEMMA 3.2 (Markovian property). Let $j \in \mathbb{N}$ and let $u_{0}, \ldots, u_{j} \in \mathbb{Z}^{2}$. Suppose that the probability of the event $\left(\gamma_{0}, \ldots, \gamma_{j}\right)=\left(u_{0}, \ldots, u_{j}\right)$ is positive. Conditioned on this event, the following hold:

(i) The paths $\Gamma^{1}, \ldots, \Gamma^{j}$ and $\Gamma\left[0, n_{j}\right]$ are conditionally independent.

(ii) For $k \in\{1, \ldots, j\}$, the conditional law of $\Gamma^{k}$ is that of a simple random walk in $D_{k-1}$ started from $u_{k}$ and conditioned to leave $D_{k-1}$ through the edge $\left[u_{k}, u_{k-1}\right]$.

(iii) The conditional law of $\Gamma\left[0, n_{j}\right]$ is that of a simple random walk started from 0 conditioned to leave $D_{j}$ at $u_{j}$, and $\gamma[j, \ell]$ is the loop erasure of the time reversal of $\Gamma\left[0, n_{j}\right]$.

PROOF. Since $\gamma$ is the loop erasure of the reversal of $\Gamma$, the event $\left(\gamma_{0}, \ldots, \gamma_{j}\right)=\left(u_{0}, \ldots, u_{j}\right)$ is equivalent to the statement that, for each $k=$ $0,1, \ldots, j-1$, the first hit of $\Gamma$ to $\left\{u_{0}, \ldots, u_{k}\right\} \cup \partial D$ is through the edge $\left[u_{k+1}, u_{k}\right]$. Let $\tau_{k}:=\min \left\{n: \Gamma(n) \in\left\{u_{0}, \ldots, u_{k}\right\} \cup \partial D\right\}, k=0, \ldots, j$. The strong Markov property of $\Gamma$ with the stopping times $\tau_{k}$ now implies the lemma.

The following simple lemma will also be needed.

Lemma 3.3 (Expected visits). Suppose that $v \in V(D)$ and that $u_{0}$ and $u_{1}$ are two vertices satisfying $\mathbf{P}\left[\gamma_{0}=u_{0}, \gamma_{1}=u_{1}\right]>0$. Conditioned on $\gamma_{0}=u_{0}$ and $\gamma_{1}=u_{1}$, the expected number of visits to $v$ by $\Gamma^{1}$ is $G\left(u_{1}, v\right) H\left(v, u_{1}\right)$.

Here $G(u, v)$ denotes the discrete Green's function, that is, the expected number of visits to $v$ by a simple random walk started at $u$, which is stopped on exiting $D$.

PROOF OF LEMmA 3.3. Let $X$ be a simple random walk from $u_{1}$ stopped on exiting $D$ and let $k$ be the last time such that $X(k)=u_{1}$. Then $\Gamma^{1}$ conditioned on $\gamma_{0}=u_{0}$ and $\gamma_{1}=u_{1}$ has the same distribution as $X$ conditioned on $X(k+1)=u_{0}$. But the path $j \mapsto X(k+j)$ is independent from $X[0, k]$. Consequently, the expected number of visits of $X$ to $v$ conditioned on $X(k+1)=u_{0}$ is equal to the expected number of visits to $v$ of $X[0, k]$. The lemma follows. 
3.2. The core argument. We keep the previous notation and also use the conformal maps $\psi_{j}: D_{j} \rightarrow \mathbb{U}$ satisfying $\psi_{j}(0)=0$ and $\psi_{j}^{\prime}(0)>0$. Set $U_{j}:=$ $\psi_{j}\left(\gamma_{j}\right)$ and $U:=U_{0}$. Note that $\gamma$ can also be viewed as a continuously growing simple curve from $\partial D$ to 0 and therefore can be represented by Loewner's equation. Let $W:[0, \infty) \rightarrow \partial \mathbb{U}$ denote the (unique) continuous function such that solving the radial Loewner equation with driving function $W(t)$ gives the path $\gamma$. Note that $U_{j}=W\left(t_{j}\right)$, where $t_{j}$ is the continuous capacity of $\gamma[0, j]$ from 0 in $D$ (i.e., the capacity of $\psi(\gamma[0, j])$ from 0 in $\mathbb{U})$. We denote by $(\vartheta(t), t \geq 0)$ the continuous real-valued function with $\vartheta(0)=0$ such that $W(t)=W(0) \exp (i \vartheta(t))$. We also define $\Delta_{j}=\vartheta\left(t_{j}\right)$, so that $U_{j}=U \exp \left(i \Delta_{j}\right)$.

PROPOSITION 3.4 (The key estimate). There exists a positive constant $C$ such that, for all small positive $\delta$, there exists $r_{0}=r_{0}(\delta)$ such that the following holds. Let $D \in \mathfrak{D}$ satisfy $\operatorname{rad}_{0}(D)>r_{0}$. For every $u_{0} \in V_{\partial}(D)$ with $\mathbf{P}\left[\gamma_{0}=u_{0}\right]>0$, let $\gamma$ denote the random path from $u_{0}$ to 0 obtained by loop erasure of the time reversal of a simple random walk from 0 to $\partial D$ conditioned to hit $\partial D$ in $u_{0}$. Let

$$
m:=\min \left\{j \geq 1: t_{j} \geq \delta^{2} \text { or }\left|\Delta_{j}\right| \geq \delta\right\},
$$

where $\Delta_{j}$ and $t_{j}$ are as described above. Then

$$
\left|\mathbf{E}\left[\Delta_{m}\right]\right| \leq C \delta^{3}
$$

and

$$
\left|\mathbf{E}\left[\Delta_{m}^{2}\right]-2 \mathbf{E}\left[t_{m}\right]\right| \leq C \delta^{3} .
$$

Recall that Lemma 3.1 says that $\gamma$ has the same distribution as the chronological loop erasure of a random walk from 0 to $\partial D$ conditioned to hit $\partial D$ at $u_{0}$.

Here is a rough sketch of the proof. Let $v \in V(D)$ satisfy

$$
\operatorname{rad}_{0}(D) / 200<|v|<\operatorname{rad}_{0}(D) / 5 \text {. }
$$

Let $h_{0}^{+}$denote the number of visits to $v$ by $\Gamma$. (This is the quantity which we referred to in Section 1 as the "observable.") The proof is based on estimating the two sides of the equality

$$
\mathbf{E}\left[h_{0}^{+}\right]=\mathbf{E}\left[\mathbf{E}\left[h_{0}^{+} \mid \gamma[0, m]\right]\right] .
$$

The estimate for the right-hand side will involve the distribution of $t_{m}$ and $\Delta_{m}$. We get the two relations (3.1) and (3.2) by considering two different choices for such a $v$.

The estimates for the two sides of (3.4) are rather straightforward. Basically, each side is translated into expressions involving the Green's functions $G_{j}$ and the hitting probabilities $H_{j}$. These are then translated into analytic quantities using (2.6). Earlier versions of the proof required other estimates, somewhat more delicate, in addition to (2.6). Fortunately, it turned out that (2.6) is sufficient. Since 
we came across several different variants for the proof, based on choosing different observables, it may be said that the proof is inevitable, rather than accidental (and this also applies to Theorem 1.3). Basically, the reason the proof works is that the expected number of visits to $v$ in $\bigcup_{j=1}^{m} \Gamma^{j}$ given $\gamma[0, m]$ can be estimated rather well given the rough geometry of $\gamma[0, m]$ in a scale much coarser than the scale of the grid. Similarly, it is important that $\mathbf{E}\left[h_{0}^{+}\right]$can be estimated given the rough geometry of $D$, but this fact is not surprising.

In the following, we abbreviate the Green's functions and hitting probabilities in $D_{j}$ by $G_{j}:=G_{D_{j}}$ and $H_{j}:=H_{D_{j}}$. The following lemma will be needed.

LEMmA 3.5 (Green's function bounds). There is a constant $C>0$ such that, for every $D \in \mathfrak{D}$ and $v \in V(D)$ satisfying (3.3),

$$
1 / C \leq G_{D}(0, v) \leq C
$$

holds. Also, given $\delta>0$, there is an $r=r(\delta)$ such that, if $\operatorname{rad}_{0}(D)>r$, then, with the notation of Proposition 3.4,

$$
G_{0}(0, v)-G_{m}(0, v) \leq C \delta^{2} .
$$

Proof OF Proposition 3.4. Since $t_{m-1}<\delta^{2}$, it follows from the Koebe $1 / 4$ theorem that $\operatorname{rad}_{0}\left(D_{m-1}\right)>\operatorname{rad}_{0}(D) / 5 \geq r_{0} / 5$ if $\delta$ is small. (Apply [35], Corollary 1.4 , with $z=0$ to $\psi_{0}^{-1}$ and $\psi_{m-1}^{-1}$.) Moreover, the continuous harmonic measure in $D$ at 0 of any edge $e$ with a vertex on $\partial D$ can be made arbitrarily small by requiring $\operatorname{rad}_{0}(D)$ to be large. [A Brownian motion started at 0 has probability going to 1 to surround the disk $\operatorname{rad}_{0}(D) \mathbb{U}$ before hitting $e$, as $\operatorname{rad}_{0}(D) \rightarrow \infty$.] By the conformal invariance of the harmonic measure, this implies that the diameter of $\psi(e)$ can be made arbitrarily small. Applying this to the domains $D_{j}$ and using Lemma 2.1, we see that we may take $r_{0}$ large enough so that, for all $j<m$, for all $t \in\left[t_{j}, t_{j+1}\right],\left|\vartheta(t)-\vartheta\left(t_{j}\right)\right| \leq \delta^{3}$ and $\left|t_{j+1}-t_{j}\right| \leq \delta^{3}$. In particular, $t_{m} \leq \delta^{2}+\delta^{3}$ and $\left|\Delta_{m}\right| \leq \delta+\delta^{3}$. We also require $r_{0} / 8$ to be larger than the $r(\delta)$ of Lemma 3.5.

Suppose $v \in V(D)$ satisfies (3.3). Set $Z_{j}:=\psi_{j}(v)$ and $Z:=Z_{0}$. For each $j \in\{1, \ldots, \ell\}$, let $h_{j}$ denote the number of visits to $v$ by $\Gamma^{j}$. Also let

$$
h_{j}^{+}:=\sum_{k=j+1}^{\ell} h_{k},
$$

which is the number of visits of $v$ by $\Gamma\left[0, n_{j}\right]$. Let $\lambda_{j}:=\lambda\left(v, \gamma_{j} ; D_{j}\right)$, where $\lambda\left(v, v^{\prime} ; D_{j}\right)$ is as in (2.5). Since, conditionally on $\gamma[0, j], \Gamma\left[0, n_{j}\right]$ is a random walk in $D_{j}$ conditioned to leave $D_{j}$ at $\gamma_{j}$,

$$
\mathbf{E}\left[h_{j}^{+} \mid \gamma[0, j]\right]=\frac{G_{j}(0, v) H_{j}\left(v, \gamma_{j}\right)}{H_{j}\left(0, \gamma_{j}\right)}
$$


and Proposition 2.2 [together with (3.5)] implies that if $r_{0}(\delta)$ is sufficiently large, then, for every $j \in\{0,1, \ldots, m\}$,

$$
\mathbf{E}\left[h_{j}^{+} \mid \gamma[0, j]\right]=G_{j}(0, v) \lambda_{j}+O\left(\delta^{3}\right) .
$$

[This $O(\cdot)$ notation is shorthand for the statement that there is an absolute constant $C$ such that $\left|\mathbf{E}\left[h_{j}^{+} \mid \gamma[0, j]\right]-G_{j}(0, v) \lambda_{j}\right| \leq C \delta^{3}$. We freely use this shorthand below.] In particular,

$$
\mathbf{E}\left[\sum_{j=1}^{m} h_{j}\right]=\mathbf{E}\left[h_{0}^{+}-h_{m}^{+}\right]=\mathbf{E}\left[G_{0}(0, v) \lambda_{0}-G_{m}(0, v) \lambda_{m}\right]+O\left(\delta^{3}\right) .
$$

We will now get a different approximation for the left-hand side. Applying Lemma 3.3 to the domain $D_{j-1}$ gives

$$
\mathbf{E}\left[h_{j} \mid \gamma[0, j]\right]=G_{j-1}\left(\gamma_{j}, v\right) H_{j-1}\left(v, \gamma_{j}\right) .
$$

Proposition 2.2 implies that, for $r_{0}(\delta)$ large enough,

$$
\mathbf{E}\left[h_{j} \mid \gamma[0, j]\right]=\left(\lambda_{j-1}+O(\delta)\right) G_{j-1}\left(\gamma_{j}, v\right) H_{j-1}\left(0, \gamma_{j}\right) .
$$

Considering the same simple random walk starting at 0 and stopped when it exits $D_{j}$ or $D_{j-1}$ shows that

$$
G_{j-1}(0, v)-G_{j}(0, v)=H_{j-1}\left(0, \gamma_{j}\right) G_{j-1}\left(\gamma_{j}, v\right) .
$$

We now derive an a priori bound on $\max \left\{\left|\lambda_{j}-\lambda_{m}\right|: j \leq m\right\}$. Recall that

$$
\lambda_{j}-\lambda_{0}=\operatorname{Re}\left(\frac{U_{j}+Z_{j}}{U_{j}-Z_{j}}-\frac{U+Z}{U-Z}\right) .
$$

But $\left|U_{j}-U\right| \leq O(\delta)$ for $j \leq m$, and Loewner's equation shows that

$$
\begin{aligned}
\forall j \leq m, \quad Z_{j} & =Z+t_{j} Z \frac{U+Z}{U-Z}+t_{j} O(\delta) \\
& =Z+t_{j} Z \frac{U+Z}{U-Z}+O\left(\delta^{3}\right),
\end{aligned}
$$

and, in particular, $Z_{j}=Z+O\left(\delta^{2}\right)$. (The equation blows up when $|U-Z|$ is small, and such estimates would not be valid in such a situation. However, this is not a problem here. First, $\psi_{0}^{\prime}(0) \leq 1 / \operatorname{rad}_{0}(D)$ by the Schwarz lemma applied to the restriction of $\psi_{0}$ to $\operatorname{rad}_{0}(D) \mathbb{U}$. Now the Koebe $1 / 4$ theorem (the case $z=0$ in the left-hand inequality in [35], Corollary 1.4) gives $\psi_{0}^{-1}((4 / 5) \mathbb{U}) \supset(1 / 4)\left|\psi_{0}^{\prime}(0)\right|^{-1}(4 / 5) \mathbb{U} \supset\left(\operatorname{rad}_{0}(D) / 5\right) \mathbb{U}$. In particular, $|Z|=$ $\left|\psi_{0}(v)\right| \leq 4 / 5$ by (3.3). Since $t_{m}=O\left(\delta^{2}\right)$, it is clear that if $\delta$ is small and one starts flowing from $Z$ according to Loewner's equation, it is impossible for $Z$ to get close to $\partial \mathbb{U}$ up to time $t_{m}$.) Thus, we get our bound,

$$
\forall j \leq m, \quad\left|\lambda_{j}-\lambda_{m}\right| \leq O(\delta) .
$$


Using (3.8), this implies

$$
\mathbf{E}\left[h_{j} \mid \gamma[0, j]\right]=\left(\lambda_{m}+O(\delta)\right) G_{j-1}\left(\gamma_{j}, v\right) H_{j-1}\left(0, \gamma_{j}\right) .
$$

Now applying (3.9) yields

$$
\mathbf{E}\left[\sum_{j=1}^{m} h_{j}\right]=\mathbf{E}\left[\left(\lambda_{m}+O(\delta)\right)\left(G_{0}(0, v)-G_{m}(0, v)\right)\right]
$$

and hence (3.6) implies

$$
\mathbf{E}\left[\sum_{j=1}^{m} h_{j}\right]=\mathbf{E}\left[\lambda_{m}\left(G_{0}(0, v)-G_{m}(0, v)\right)\right]+O\left(\delta^{3}\right) .
$$

Comparing with (3.7) gives $G_{0}(0, v) \mathbf{E}\left[\lambda_{m}-\lambda_{0}\right]=O\left(\delta^{3}\right)$, and hence (3.5) implies

$$
\mathbf{E}\left[\lambda_{m}-\lambda_{0}\right]=O\left(\delta^{3}\right)
$$

[The reader may wonder about the apparent miracle happening here, namely, that $\lambda_{j}$ turns out to be "almost" a martingale. In fact, this is not important for identifying the scaling limit. If the right-hand side in (3.12) turned out to be any other explicit quantity, up to $\delta^{3}$ error terms, the proof would still work, but give a different limiting process. In Remark 3.6 below, we give a short proof of (3.12) and further comments.]

Recall that this equation is valid uniformly over all choices of $v$. We now Taylorexpand $\lambda_{m}-\lambda_{0}$ with respect to $U_{m}-U$ and $Z_{m}-Z$, up to $O\left(\delta^{3}\right)$ error terms. As we have seen, $U_{m}-U=O(\delta)$ and $Z_{m}-Z=O\left(\delta^{2}\right)$, and hence only the firstorder derivative with respect to $Z_{m}-Z$ and the first two derivatives with respect to

$$
U_{m}-U=\left(e^{i \Delta_{m}}-1\right) U=i U \Delta_{m}-U \Delta_{m}^{2} / 2+O\left(\delta^{3}\right)
$$

come into play (the mixed derivatives can be ignored). Using (3.10) and (3.11), we get

$$
\lambda_{m}-\lambda_{0}=\Delta_{m} \operatorname{Im}\left(\frac{2 Z U}{(U-Z)^{2}}\right)+\left(2 t_{m}-\Delta_{m}^{2}\right) \operatorname{Re}\left(\frac{Z U(U+Z)}{(U-Z)^{3}}\right)+O\left(\delta^{3}\right),
$$

and therefore (3.12) gives

$$
\operatorname{Im}\left(\frac{2 Z U}{(U-Z)^{2}}\right) \mathbf{E}\left[\Delta_{m}\right]+\operatorname{Re}\left(\frac{Z U(U+Z)}{(U-Z)^{3}}\right) \mathbf{E}\left[2 t_{m}-\Delta_{m}^{2}\right]=O\left(\delta^{3}\right) .
$$

We claim that when $r_{0}(\delta)$ is large enough we may find $v_{1}, v_{2} \in V(D)$ in the range (3.3) satisfying $\left|\psi\left(v_{1}\right)-U / 30\right|<\delta^{3}$ and $\left|\psi\left(v_{2}\right)-i U / 30\right|<\delta^{3}$. Indeed, by Theorem 1.3 and Corollary 1.4 from [35], for every $R \in(0,1)$, there is a $C=C(R)<\infty$ such that $\left|\psi^{\prime}(z)\right| \leq C / \operatorname{rad}_{z}(D)$ and $\operatorname{rad}_{z}(D) \geq C^{-1} \operatorname{rad}_{0}(D)$ hold for all $z \in \psi^{-1}(R \mathbb{U})$. Let $v_{1}$ be a vertex closest to $\psi^{-1}(U / 30)$. By integrating 
the above bound on $\psi^{\prime}$ along the line segment from $\psi^{-1}(U / 30)$ to $v_{1}$ (whose length is less than 1 ), we get $\left|\psi\left(v_{1}\right)-U / 30\right|<\delta^{3}$ if $\operatorname{rad}_{0}(D)$ is large enough. Another application of Theorem 1.3 and Corollary 1.4 from [35] now shows that $v_{1}$ satisfies (3.3). An entirely similar argument produces $v_{2}$.

Consequently, (3.13) holds with $Z \in\{U / 30, i U / 30\}$. Plugging in these two values for $Z$ produces two linearly independent equations in the variables $\mathbf{E}\left[2 t_{m}-\Delta_{m}^{2}\right]$ and $\mathbf{E}\left[\Delta_{m}\right]$ and thereby proves (3.1) and (3.2).

REMARK 3.6. Here is another proof of (3.12). Given a vertex $v \in V(D)$, let $\beta=\left(\beta_{0}, \beta_{1}, \ldots\right)$ denote the loop erasure of the reversal of the simple random walk $\Gamma_{v}$ started from $v$ and stopped on exiting $D$ (i.e., the analog of $\gamma$, but starting from $v$ instead of 0$)$. Abbreviate $\gamma^{n}:=\left(\gamma_{0}, \ldots, \gamma_{n}\right)$ and similarly $\beta^{n}:=\left(\beta_{0}, \ldots, \beta_{n}\right)$. For a sequence of vertices $u=\left(u_{0}, u_{1}, \ldots, u_{n}\right)$, let $a_{n}(u):=\mathbf{P}\left[\gamma^{n}=u\right]$ and $b_{n}(u):=\mathbf{P}\left[\beta^{n}=u\right]$. Set $M_{n}:=b_{n}\left(\gamma^{n}\right) / a_{n}\left(\gamma^{n}\right)$. (In other words, $M_{n}$ is the Radon-Nikodym derivative of the law of $\beta^{n}$ with respect to the law of $\gamma^{n}$.) It is easy to verify that $M_{n}$ is a martingale:

$$
\mathbf{E}\left[M_{n+1} \mid \gamma^{n}\right]=\sum_{w} \frac{b_{n+1}\left(\gamma^{n} w\right)}{a_{n+1}\left(\gamma^{n} w\right)} \frac{a_{n+1}\left(\gamma^{n} w\right)}{a_{n}\left(\gamma^{n}\right)}=\frac{\sum_{w} b_{n+1}\left(\gamma^{n} w\right)}{a_{n}\left(\gamma^{n}\right)}=M_{n} .
$$

Lemma 3.2 implies that $M_{n}=H_{n}\left(v, \gamma_{n}\right) / H_{n}\left(0, \gamma_{n}\right)$, since, on the event that $\Gamma_{v}$ and $\Gamma$ first hit $\left\{u_{0}, \ldots, u_{n}\right\} \cup \partial D$ at $u_{n}$, we may couple them to agree after that first visit to $u_{n}$. Now (2.6) implies (3.12).

Although this proof is shorter than the first proof of (3.12), it is harder to motivate and less natural. For this reason, we chose to stress the first proof.

Let us finally note that (as opposed to the martingale that shows up in the analysis of the UST Peano curve), the quantity corresponding to this martingale in the scaling limit is unbounded and converges almost surely to 0 (it is not uniformly integrable), so that it cannot be interpreted as a conditional probability or a conditional expectation. Correspondingly, in the discrete setting, $M_{n}$ is very large when the path hits $v$ (if it does) and $M_{n}$ is very small when the path hits 0 .

3.3. Recognizing the driving process. The objective in this section is to show that $W$ of the previous section is close to a time-scaled Brownian motion on the unit circle.

THEOREM 3.7 (Driving process convergence). For every $T>0$ and $\epsilon>0$, there is an $r_{1}=r_{1}(\epsilon, T)>0$ such that, for all $D \in \mathfrak{D}$ with $\operatorname{rad}_{0}(D)>r_{1}$, there is a coupling of $\gamma$ with Brownian motion $B(t)$ starting at a random uniform point in $[0,2 \pi]$ such that

$$
\mathbf{P}[\sup \{|\vartheta(t)-B(2 t)|: t \in[0, T]\}>\epsilon]<\epsilon .
$$


Recall that a coupling of two random variables (or random processes) $A$ and $B$ is a probability space with two random variables $A^{\prime}$ and $B^{\prime}$, where $A^{\prime}$ has the same distribution as $A$ and $B^{\prime}$ has the same distribution as $B$. In the above statement (as is customary), we do not distinguish between $A$ and $A^{\prime}$ and between $B$ and $B^{\prime}$.

In order to deduce this theorem from Proposition 3.4, we will use the Skorohod embedding theorem, which is one of the standard tools for proving convergence to Brownian motion (one could work out a more direct proof but the following proof seems cleaner).

LEMMA 3.8 (Skorohod embedding). If $\left(M_{n}\right)_{n \leq N}$ is an $\left(\mathcal{F}_{n}\right)_{n \leq N}$ martingale, with $\left\|M_{n}-M_{n-1}\right\|_{\infty} \leq 2 \delta$ and $M_{0}=0$ a.s., then there are stopping times $0=\tau_{0} \leq \tau_{1} \leq \cdots \leq \tau_{N}$ for standard Brownian motion $\left(B_{t}, t \geq 0\right)$ such that $\left(M_{0}, M_{1}, \ldots, M_{N}\right)$ and $\left(B_{\tau_{0}}, B_{\tau_{1}}, \ldots, B_{\tau_{N}}\right)$ have the same law. Moreover, one can impose, for $n=0,1, \ldots, N-1$,

$$
\mathbf{E}\left[\tau_{n+1}-\tau_{n} \mid B\left[0, \tau_{n}\right]\right]=\mathbf{E}\left[\left(B_{\tau_{n+1}}-B_{\tau_{n}}\right)^{2} \mid B\left[0, \tau_{n}\right]\right]
$$

and

$$
\tau_{n+1} \leq \inf \left\{t \geq \tau_{n}:\left|B_{t}-B_{\tau_{n}}\right| \geq 2 \delta\right\} .
$$

The proof can be found in many probability textbooks, including [9] and [37]. Often, it is stated for just one random variable $M_{1}$; for a statement in terms of martingales, see, for instance, [8] and [43]. The relation (3.15) is not stated explicitly in these references (since the assumption that the increments of $M_{n}$ are bounded is weakened), but is a consequence of the proof. It can also be derived a posteriori from $\mathbf{E}\left[\tau_{n+1}-\tau_{n}\right]=\mathbf{E}\left[\left(M_{n+1}-M_{n}\right)^{2}\right]<\infty$, since the expected time for Brownian motion started outside an interval to hit the interval is infinite.

PROOF OF THEOREM 3.7. Since the hitting measure of a simple random walk from 0 is close to the hitting measure for Brownian motion when $\operatorname{rad}_{0}(D)$ is large (see, e.g., Section 5), it is clear that $W(0)$ is nearly uniform in $\partial \mathbb{U}$. It is therefore enough to show that $\vartheta(t / 2)$ is close to standard Brownian motion.

Assume, without loss of generality, that $T \geq 1$. Pick $\delta=\delta(\epsilon, T)>0$ small. Let $r_{0}$ be as in Proposition 3.4 and take $r_{1}:=8 \exp (20 T) r_{0}$. Let $\gamma^{t}$ denote the initial segment of $\gamma$ such that $\psi_{D}\left(\gamma^{t}\right)$ has capacity $t$ from 0 . By the Schwarz lemma, $\psi_{D}^{\prime}(0) \leq \operatorname{rad}_{0}(D)^{-1}$. Therefore, the Koebe $1 / 4$ theorem implies $\operatorname{rad}_{0}\left(D \backslash \gamma^{t}\right) \geq$ $\exp (-t) \operatorname{rad}_{0}(D) / 4$. Hence, if $\operatorname{rad}_{0}(D) \geq r_{1}$, Proposition 3.4 is valid not only for the initial domain $D$, but also for the domain $D$ slitted by subarcs of $\gamma$, up to capacity $20 T$.

As in Proposition 3.4, define $m$ to be the first $j=1,2, \ldots$ such that $\left|\Delta_{j}\right| \geq \delta$ or $t_{j} \geq \delta^{2}$. Set $m_{0}:=0, m_{1}:=m$ and inductively let $m_{n+1}$ be the first $j \geq m_{n}+1$ such that $\left|\Delta_{j}-\Delta_{m_{n}}\right| \geq \delta$ or $t_{j}-t_{m_{n}} \geq \delta^{2}$, whichever happens first. Let $\widetilde{\mathcal{F}}_{n}$ denote the $\sigma$-field generated by $\gamma\left[0, m_{n}\right]$. Set

$$
N:=\left\lceil 10 T \delta^{-2}\right\rceil \text {. }
$$


Our choice of $r_{1}$ ensures that $t_{j+1}-t_{j} \leq 2 \delta^{2}$ for all $j<N$ and that $t_{N} \leq 20 T$. Hence, Proposition 3.4 holds for all domains $D_{m_{n}}$ with $n<N$. Applying clause (iii) of Lemma 3.2 therefore gives

$$
\mathbf{E}\left[\Delta_{m_{n+1}}-\Delta_{m_{n}} \mid \mathcal{F}_{n}\right]=O\left(\delta^{3}\right)
$$

and

$$
\mathbf{E}\left[\left(\Delta_{m_{n+1}}-\Delta_{m_{n}}\right)^{2} \mid \mathcal{F}_{n}\right]=2 \mathbf{E}\left[t_{m_{n+1}}-t_{m_{n}} \mid \mathcal{F}_{n}\right]+O\left(\delta^{3}\right) .
$$

For $n \leq N$, set

$$
M_{n}:=\sum_{j=0}^{n-1}\left(\Delta_{m_{j+1}}-\Delta_{m_{j}}-\mathbf{E}\left[\Delta_{m_{j+1}}-\Delta_{m_{j}} \mid \mathcal{F}_{j}\right]\right) .
$$

Clearly, $M_{0}, \ldots, M_{N}$ is a martingale for $\mathcal{F}_{0}, \ldots, \mathcal{F}_{N}$. The definition of $m_{n}$ and the choice of $r_{1}$ imply that $\left\|M_{n+1}-M_{n}\right\|_{\infty} \leq 2 \delta$.

By Lemma 3.8, we may couple $\left(M_{0}, \ldots, M_{N}\right)$ with a standard Brownian motion with stopping times $\tau_{0} \leq \tau_{1} \leq \cdots \leq \tau_{N}$ such that $B_{\tau_{n}}=M_{n}$ and (3.14) hold. Extend the coupling to include $\gamma$ (this clearly can be done).

Note that the definition of $t_{m_{n}}$ and (3.15) ensure that, for all $n<N$,

$$
\begin{array}{r}
\sup \left\{\left|B_{t}-B_{\tau_{n}}\right|: t \in\left[\tau_{n}, \tau_{n+1}\right]\right\} \leq 2 \delta, \\
\sup \left\{\left|\vartheta(t)-\Delta_{t_{m_{n}}}\right|: t \in\left[t_{m_{n}}, t_{m_{n+1}}\right]\right\} \leq 2 \delta
\end{array}
$$

and (3.16) shows that

$$
\sup \left\{\left|\Delta_{t_{m}}-M_{n}\right|: n \leq N\right\}=O\left(\delta^{3} N\right)=O(\delta T) .
$$

Hence, as $M_{n}=B_{\tau_{n}}$ and $B_{t}$ is a.s. continuous, it remains to relate the capacities $t_{m_{n}}$ with the stopping times $\tau_{n}$ and verify that $t_{m_{N}}>T$ with high probability. For this purpose, define

$$
Y_{n}=\sum_{j=0}^{n-1}\left(M_{j+1}-M_{j}\right)^{2}
$$

We first show that $Y_{n}$ is close to $2 t_{m_{n}}$. Let $Z_{n}:=Y_{n}-2 t_{m_{n}}$. By (3.18) and (3.16), we have, for $n<N,\left|M_{n+1}-M_{n}-\Delta_{t_{m_{n+1}}}+\Delta_{t_{m_{n}}}\right|=O\left(\delta^{3}\right)$. This implies $\left|M_{n+1}-M_{n}\right|=O(\delta)$ and hence also

$$
Y_{n+1}-Y_{n}=\left(M_{n+1}-M_{n}\right)^{2}=\left(\Delta_{t_{m_{n+1}}}-\Delta_{t_{m_{n}}}\right)^{2}+O\left(\delta^{4}\right) .
$$

Consequently, (3.17) gives

$$
\mathbf{E}\left[Z_{n+1}-Z_{n} \mid \mathcal{F}_{n}\right] \leq O\left(\delta^{3}\right) .
$$

From the fact that the increments of $t_{m_{n}}$ and those of $Y_{n}$ are bounded by $O\left(\delta^{2}\right)$, 
we also have $\mathbf{E}\left[\left(Z_{n+1}-Z_{n}\right)^{2} \mid \mathcal{F}_{n}\right] \leq O\left(\delta^{4}\right)$. Set $Z_{n}^{\prime}:=Z_{n}-\sum_{j=1}^{n} \mathbf{E}\left[Z_{j}-\right.$ $\left.Z_{j-1} \mid \mathcal{F}_{j-1}\right]$. Since this is an $\mathscr{F}_{n}$-martingale, we have $\mathbf{E}\left[Z_{N}^{\prime}{ }^{2}\right]=\sum_{j=1}^{N} \mathbf{E}\left[\left(Z_{j}^{\prime}-\right.\right.$ $\left.\left.Z_{j-1}^{\prime}\right)^{2}\right]$ and the above estimates give $\mathbf{E}\left[Z_{N}^{\prime}{ }^{2}\right]=O\left(N \delta^{4}\right)$. Assuming $N \delta^{3}<$ $\delta^{1 / 2} / 2$, without loss of generality, and applying Doob's maximal inequality (see [37], II.1.7) for $L^{2}$-martingales to $Z_{n}^{\prime}$, we get

$$
\mathbb{P}\left[\max _{n \leq N}\left|Y_{n}-2 t_{m_{n}}\right|>\delta^{1 / 2}\right]=O\left(N \delta^{3}\right)=O(T \delta) .
$$

By the definition of the $t_{m_{n}}$, we have $Y_{n+1}-Y_{n}+t_{m_{n+1}}-t_{m_{n}} \geq \delta^{2}$. Summing gives $Y_{N}+t_{m_{N}} \geq N \delta^{2} \geq 10 T$. Therefore, (3.21) implies

$$
\mathbf{P}\left[t_{m_{N}}<2 T\right]=O(T \delta) .
$$

We now show that with high probability $\tau_{n}$ is also close to $Y_{n}$ for every $n \leq N$. By (3.15), it is clear that $\mathbf{E}\left[\left(\tau_{n+1}-\tau_{n}\right)^{2} \mid B\left[0, \tau_{n}\right]\right]=O\left(\delta^{4}\right)$ and therefore

$$
\mathbf{E}\left[\left(\left(\tau_{n+1}-Y_{n+1}\right)-\left(\tau_{n}-Y_{n}\right)\right)^{2} \mid B\left[0, \tau_{n}\right]\right]=O\left(\delta^{4}\right) .
$$

Also, (3.14) gives

$$
\mathbf{E}\left[\left(\tau_{n+1}-Y_{n+1}\right)-\left(\tau_{n}-Y_{n}\right) \mid B\left[0, \tau_{n}\right]\right]=0 .
$$

Doob's inequality therefore implies

$$
\mathbf{P}\left[\max _{n \leq N}\left|\tau_{n}-Y_{n}\right|>\delta^{1 / 2}\right]=O(T \delta) .
$$

Combining this with (3.21) leads to

$$
\mathbf{P}\left[\max _{n \leq N}\left|\tau_{n}-2 t_{m_{n}}\right|>\delta^{1 / 2}\right]=O(T \delta) .
$$

Since $B_{t}$ is a.s. continuous, together with (3.22), (3.19) and (3.20), this completes the proof.

3.4. Convergence with respect to a stronger topology. Theorem 3.7 provides a kind of convergence of the loop-erased random walk to $\mathrm{SLE}_{2}$. As we will see in this section, this kind of convergence suffices, for example, to show that the scaling limit with respect to the Hausdorff metric of the union of $\partial \mathbb{U}$ and the LERW in $\mathbb{U}$ is the union of $\partial \mathbb{U}$ and the $\mathrm{SLE}_{2}$ path.

Let $\alpha:[0,1] \rightarrow \mathbb{C}$ and $\beta:[0,1] \rightarrow \mathbb{C}$ be two continuous paths. Define

$$
\rho(\alpha, \beta):=\inf _{\phi \in \Phi} \sup _{t \in[0,1]}|\alpha(t)-\beta \circ \phi(t)|,
$$

where $\Phi$ is the collection of all monotone nondecreasing continuous maps from $[0,1]$ onto $[0,1]$. It is an easy well-known fact that $\rho$ is a metric on equivalence classes of paths, where two paths $\alpha$ and $\beta$ are equivalent if $\alpha \circ \phi_{1}=\beta \circ \phi_{2}$, where 
$\phi_{1}, \phi_{2} \in \Phi$. Since $\rho(\alpha, \beta)$ does not depend on the particular parameterization of $\alpha$ or $\beta$, the metric $\rho$ is also defined for paths on intervals other than $[0,1]$.

To explain our present goal, let us point out that there is a sequence of paths $\alpha_{n}$ from 1 to 0 in $\overline{\mathbb{U}}$ such that their Loewner driving functions $W_{n}(t)$ converge uniformly to the constant 1 but $\alpha_{n}$ does not converge to the path $\alpha(t)=1-t$, $t \in[0,1]$, in the metric $\rho$, although the driving function for $\alpha$ (reparameterized by capacity) is the constant 1 . For example, we may take $\alpha_{n}$ as the polygonal path through the points $a_{1}, b_{1}+i n^{-2}, a_{2}, b_{2}-i n^{-2}, a_{3}, b_{3}+i n^{-2}, \ldots, a_{\lfloor n / 2\rfloor}, 0$, where $a_{j}:=1-n^{-1}+(j n)^{-1}$ and $b_{j}:=1-j / n$.

THEOREM 3.9 (LERW image in $\overline{\mathbb{U}}$ converges). For any sequence $D_{n} \in \mathfrak{D}$ with $\operatorname{rad}_{0}\left(D_{n}\right) \rightarrow \infty$, if $\mu_{n}$ denotes the law of $\tilde{\gamma}^{n}:=\psi_{D_{n}} \circ \gamma^{n}$, where $\gamma^{n}$ is the time reversal of the $L E R W$ from 0 to $\partial D_{n}$, then $\mu_{n}$ converges weakly (with respect to the metric $\rho$ ) to the law of the radial $\mathrm{SLE}_{2}$ path started uniformly on the unit circle.

The outline of the proof is as follows. We define a suitable family of compact subsets of the space of simple paths from $\partial \mathbb{U}$ to 0 in $\overline{\mathbb{U}}$, which we can use to show that the sequence $\mu_{n}$ is tight. (See, e.g., [9] for background on weak convergence and the notion of tightness.) This implies that a subsequence of $\mu_{n}$ converges weakly to some probability measure. Theorem 3.9 then shows that the law of $\mathrm{SLE}_{2}$ is the unique possible subsequential limit.

In order to prove tightness, we will use properties of the loop-erased random walk proved in [38]. The actual details will require some background in the geometric theory of conformal maps. In particular, some properties of extremal distance (a.k.a. extremal length) will be used. See, for example, [2] for background. The basic ideas that are used in the proof are taken from [3] and [38].

For a simply connected $D \varsubsetneqq \mathbb{C}$ containing 0 , let $\mathcal{X}_{0}(D)$ denote the space of all simple paths $\gamma:[0, \infty] \rightarrow \bar{D}$ from $\partial D$ to 0 in $\bar{D}$, which intersect $\partial D$ only at the starting point. Given a monotone nondecreasing function $\Upsilon:(0, \infty) \rightarrow(0,1]$, let $X_{\Upsilon}(D) \subset \mathcal{X}_{0}(D)$ denote the space of all simple paths $\gamma \in \mathcal{X}_{0}(D)$ such that, for every $0 \leq s_{1}<s_{2}$,

$$
\operatorname{dist}\left(\gamma\left[0, s_{1}\right] \cup \partial D, \gamma\left[s_{2}, \infty\right]\right) / \operatorname{rad}_{0}(D) \geq \Upsilon\left(\operatorname{diam}\left(\gamma\left[s_{1}, s_{2}\right]\right) / \operatorname{rad}_{0}(D)\right) .
$$

Note that whether $\gamma \in \mathcal{X}_{\Upsilon}(D)$ does not depend on the parameterization of $\gamma$ and is scaling invariant.

LEMMA 3.10 (Compactness). Let $\Upsilon:(0, \infty) \rightarrow(0,1]$ be monotone nondecreasing. Then $\mathcal{X}_{\Upsilon}(\mathbb{U})$ is compact in the topology of convergence with respect to $\rho$.

PROOF. We use an idea from [3]. For all $n \in \mathbb{N}$, let $Z_{n}$ be a finite collection of points such that the open balls $\mathscr{B}\left(z, 2^{-n}\right), z \in Z_{n}$, cover $\mathbb{U}$. Given a set $K \subset \overline{\mathbb{U}}$ 
and a point $z \in Z_{n}$, let $s(K, z, n)$ denote the diameter of $K \cap \mathscr{B}\left(z, 2^{1-n}\right)$ in the metric obtained from the Euclidean metric on the disk $\overline{\mathscr{B}\left(z, 2^{1-n}\right)}$ by collapsing the boundary $\partial \mathscr{B}\left(z, 2^{1-n}\right)$ to a single point. [In other words, this metric $d(x, y)$ is defined as $d(x, y)=\min \{|x-y|, \operatorname{dist}(x, \partial \mathscr{B})+\operatorname{dist}(y, \partial \mathscr{B})\}$, where $\mathscr{B}=$ $\mathscr{B}\left(z, 2^{1-n}\right)$.]

Fix $\gamma \in \mathcal{X}_{\Upsilon}(\mathbb{U})$. Given $t \geq 0$, let

$$
s(t)=s_{\gamma}(t):=\sum_{n \in \mathbb{N}} \sum_{z \in Z_{n}} \frac{s(\gamma[0, t], z, n)}{\left|Z_{n}\right|} .
$$

Clearly, $s_{\gamma}(t) \leq \sum_{n \geq 0} 2^{2-n}=8$, and $s:[0, \infty] \rightarrow[0, \infty)$ is continuous and strictly monotone increasing. (To verify that $s$ is strictly monotone increasing, note that, if $t_{2}>t_{1} \geq 0$, then there is some $n \in \mathbb{N}$ such that $\operatorname{dist}\left(\gamma\left(t_{2}\right), \gamma\left[0, t_{1}\right]\right) \geq 2^{2-n}$, and so $s\left(\gamma\left[0, t_{2}\right], z, n\right) \geq s\left(\gamma\left[0, t_{1}\right], z, n\right)+2^{-n}$ if $z \in Z_{n}$ satisfies $\gamma\left(t_{2}\right) \in \mathscr{B}\left(z, 2^{-n}\right)$.) Let $\hat{\gamma}(s)$ be $\gamma$ parameterized by $s$; that is, $\hat{\gamma}=\gamma \circ s^{-1}$. Let $s_{1}<s_{2}$ and set $\epsilon:=\operatorname{diam} \hat{\gamma}\left[s_{1}, s_{2}\right]>0$. Then $\operatorname{dist}\left(\hat{\gamma}\left(s_{2}\right), \hat{\gamma}\left[0, s_{1}\right] \cup \partial \mathbb{U}\right) \geq \Upsilon(\epsilon)$. By the argument for strict monotonicity given above, this shows that $s_{2}-s_{1} \geq 2^{-n} /\left|Z_{n}\right|$, where $n:=\min \left\{k \in \mathbb{N}: 2^{2-k} \leq \Upsilon(\epsilon)\right\}$. Therefore, $\hat{\gamma}$ satisfies an equicontinuity estimate. By the Arzela-Ascoli theorem, it follows that the closure of $\mathcal{X}_{\Upsilon}(\mathbb{U})$ is compact in the $\rho$ metric. It is also clear that $\mathcal{X}_{\Upsilon}(\mathbb{U})$ is closed.

Our next goal is to use these compact sets to prove tightness, and we start by observing that the diameter is tight.

LEMma 3.11 (Diameter is tight). There are constants $c, C>0$ such that for every $D \in \mathfrak{D}$ and every $r \geq 1$ the simple random walk $\Gamma$ starting from 0 and stopped on hitting $\partial D$ satisfies

$$
\mathbf{P}\left[\operatorname{diam}(\Gamma) \geq r \operatorname{rad}_{0}(D)\right] \leq C r^{-c} .
$$

Consequently, the same estimate holds for the loop erasure $\gamma$.

The first statement is an easy well-known fact. Since the complement of $D$ is connected and unbounded, if the random walk makes a loop separating the circle $\operatorname{rad}_{0}(D) \partial \mathbb{U}$ from the circle $(r / 2) \operatorname{rad}_{0}(D) \partial \mathbb{U}$ before hitting the latter circle, then it must hit $\partial D$ before $(r / 2) \operatorname{rad}_{0}(D) \partial \mathbb{U}$. Thus, the lemma is easily proved directly and also follows from the convergence of a simple random walk to Brownian motion. A rather precise form of this estimate for the random walk, where $c=1 / 2$, is known as the discrete Beurling theorem (see [25], Theorem 2.5.2).

LEMMA 3.12 (Tameness). For every $\epsilon>0$, there is some monotone nondecreasing $\Upsilon:(0, \infty) \rightarrow(0,1]$ and some $r_{0}>0$ such that for every $D \in \mathfrak{D}$ with $\operatorname{rad}_{0}(D) \geq r_{0}$ its time-reversed loop-erased walk $\gamma=\gamma_{D}$ satisfies

$$
\mathbf{P}\left[\gamma \in \mathcal{X}_{\Upsilon}(D)\right] \geq 1-\epsilon .
$$


PROOF. The proof is essentially contained in the proof of [38], Theorem 1.1, where it is established that every subsequential scaling limit of LERW is a.s. a simple path. We will not repeat the complete proof from [38] here, but indicate how it may be adapted to yield the statement of the lemma.

Let $\epsilon>0$. Clearly, $\gamma \in \mathcal{X}_{0}(D)$. If $\gamma \notin \mathcal{X}_{\Upsilon}(D)$, then there are $0 \leq s_{1}<s_{2}<\infty$ such that the distance between $\gamma\left[0, s_{1}\right] \cup \partial D$ and $\gamma\left[s_{2}, \infty\right]$ is smaller than $\operatorname{rad}_{0}(D) \Upsilon\left(\operatorname{diam} \gamma\left[s_{1}, s_{2}\right] / \operatorname{rad}_{0}(D)\right)$. Let us first deal with the case where the distance between $\gamma\left[s_{2}, \infty\right]$ and $\partial D$ is small. Let $\Gamma$ be the walk generating the time reversal of $\gamma$, and let $t_{n}$ be the first time $t$ where the distance from $\Gamma(t)$ to $\partial D$ is smaller than $2^{-n} \operatorname{rad}_{0}(D)$ and let $\tau=\inf \{t: \Gamma(t) \in \partial D\}$. By the Markov property of $\Gamma$ at time $t_{n}$ and Lemma 3.11,

$$
\mathbf{P}\left[\operatorname{diam} \Gamma\left[t_{n}, \tau\right]>2^{-n / 2} \operatorname{rad}_{0}(D)\right] \leq C 2^{-c n / 2} .
$$

Consequently, there is an $N=N(\epsilon)$ such that with probability $1-\epsilon / 2$ for every integer $n \geq N$ we have $\operatorname{diam} \Gamma\left[t_{n}, \tau\right] \leq 2^{-n / 2} \operatorname{rad}_{0}(D)$. In this case, if $\operatorname{diam} \gamma\left[0, s_{2}\right]>2^{-n / 2} \operatorname{rad}_{0}(D)$, where $n>N$, then $\gamma\left[0, s_{2}\right]$ is not contained in $\Gamma\left[t_{n}, \tau\right]$, which implies that $\gamma\left[s_{2}, \infty\right] \subset \Gamma\left[0, t_{n}\right]$ and gives $\operatorname{dist}\left(\gamma\left[s_{2}, \infty\right], \partial D\right) \geq$ $2^{-n} \operatorname{rad}_{0}(D)$. In other words, if $\Upsilon$ satisfies

$$
\Upsilon(t)<\min \left\{t^{2}, 2^{-2 N}\right\} / 4,
$$

then with probability at least $1-\epsilon / 2$, for every $s_{1}, s_{2} \in[0, \infty]$,

$$
\operatorname{dist}\left(\partial D, \gamma\left[s_{2}, \infty\right]\right) \geq \operatorname{rad}_{0}(D) \Upsilon\left(\operatorname{diam} \gamma\left[s_{1}, s_{2}\right] / \operatorname{rad}_{0}(D)\right) .
$$

We now focus on the case where the distance between $\gamma\left[0, s_{1}\right]$ and $\gamma\left[s_{2}, \infty\right)$ is small. We shall say that $\gamma$ has a $(\beta, \alpha)$-quasi-loop if there are $0<s_{1}<s_{2}<\infty$ such that $\left|\gamma\left(s_{1}\right)-\gamma\left(s_{2}\right)\right| \leq \alpha \operatorname{rad}_{0}(D)$ but diam $\gamma\left[s_{1}, s_{2}\right] \geq \beta \operatorname{rad}_{0}(D)$. Note that if there are $0<s_{1}<s_{2}<\infty$ such that $\operatorname{dist}\left(\gamma\left[0, s_{1}\right], \gamma\left[s_{2}, \infty\right]\right)<\alpha \operatorname{rad}_{0}(D)$ and $\operatorname{diam} \gamma\left[s_{1}, s_{2}\right] \geq \beta \operatorname{rad}_{0}(D)$, then $\gamma$ has a $(\beta, \alpha)$-quasi-loop. Let $\mathcal{A}(\beta, \alpha)$ denote the event that $\gamma$ has a $(\beta, \alpha)$-quasi-loop. Assume, for the moment, that, for all $n \geq 0$,

$$
\lim _{\alpha \searrow 0} \mathbf{P}\left[\mathcal{A}\left(2^{-n}, \alpha\right)\right]=0,
$$

uniformly in $D$. Then we may take a decreasing sequence $\alpha_{n} \searrow 0$ such that $\sum_{n=1}^{\infty} \mathbf{P}\left[\mathcal{A}\left(2^{-n}, \alpha_{n}\right)\right]<\epsilon / 2$ holds for every $D \in \mathfrak{D}$. Then with probability at least $1-\epsilon / 2, \gamma$ has no $\left(2^{-n}, \alpha_{n}\right)$-quasi-loop for any $n=1,2, \ldots$ Assuming that $\Upsilon(t)<\alpha_{n}$ holds whenever $t \leq 2^{1-n}, n \in \mathbb{N}$, and $\Upsilon(t)<\alpha_{1}$ for all $t$, on this event we also have

$$
\operatorname{dist}\left(\gamma\left[0, s_{1}\right], \gamma\left[s_{2}, \infty\right]\right) \leq \operatorname{rad}_{0}(D) \Upsilon\left(\operatorname{diam} \gamma\left[s_{1}, s_{2}\right] / \operatorname{rad}_{0}(D)\right)
$$

for all $0<s_{1}<s_{2}<\infty$. If we also assume (3.23), then together with (3.24) we get $\mathbf{P}\left[\gamma \in \mathcal{X}_{\Upsilon}(D)\right] \geq 1-\epsilon$, completing the proof of the lemma. Thus, it remains to verify (3.25). 
Let $\mathcal{A}\left(z_{0}, \beta, \alpha\right)$ denote the event that there are $0<s_{1}<s_{2}<\infty$ such that $\left|\gamma\left(s_{1}\right)-\gamma\left(s_{2}\right)\right| \leq \alpha \operatorname{rad}_{0}(D), \gamma\left(s_{1}\right), \gamma\left(s_{2}\right) \in \mathcal{B}\left(z_{0}, \beta \operatorname{rad}_{0}(D) / 4\right)$ and $\operatorname{diam}\left(\gamma\left[s_{1}\right.\right.$, $\left.\left.s_{2}\right]\right) \geq \beta \operatorname{rad}_{0}(D)$. In particular, this implies that $\gamma\left[s_{1}, s_{2}\right]$ is not contained in the interior of $\mathcal{B}\left(z_{0}, \beta \operatorname{rad}_{0}(D) / 2\right)$. Assume that $8 \alpha<\beta$. By Lemma 3.11, there is an $R=R(\epsilon)>0$ such that with probability at least $1-\epsilon / 2$ we have $\gamma[0, \infty] \subset \mathscr{B}\left(0, R \operatorname{rad}_{0}(D)\right)$. There is a collection $\left\{z_{1}, z_{2}, \ldots, z_{k}\right\}$ of points such that every disk of radius $2 \alpha \operatorname{rad}_{0}(D)$ with center in $\mathcal{B}\left(0, R \operatorname{rad}_{0}(D)\right)$ is contained in one of the $k$ balls $B\left(z_{j}, \beta \operatorname{rad}_{0}(D) / 2\right), j=1,2, \ldots, k$, and we may take $k<c\left((R / \beta)^{2}+1\right)$, where $c$ is an absolute constant. On the event $\gamma[0, \infty] \subset$ $\mathscr{B}\left(0, R \operatorname{rad}_{0}(D)\right)$, we have $\mathcal{A}(\beta, \alpha) \subset \bigcup_{j=1}^{k} \mathcal{A}\left(z_{j}, \beta, \alpha\right)$. Since $\epsilon>0$ was arbitrary and $\mathbf{P}\left[\gamma[0, \infty] \subset \mathscr{B}\left(0, R \operatorname{rad}_{0}(D)\right)\right] \geq 1-\epsilon / 2$, it is therefore sufficient to show that $\mathbf{P}\left[\mathcal{A}\left(z_{j}, \beta, \alpha\right)\right] \rightarrow 0$ as $\alpha \rightarrow 0$ uniformly in $D$. The proof of this statement is given (with minor changes in the setup) in [38], Theorem 1.1.

Let $\mathcal{X}_{\Upsilon}^{r}(D)$ denote the set of paths $\gamma \in \mathcal{X}_{\Upsilon}(D)$ that are contained in the ball of radius $r \operatorname{rad}_{0}(D)$ about 0 . Given $\gamma \in X_{0}(D)$, let $\gamma^{*}:[0, \infty) \rightarrow \overline{\mathbb{U}}$ denote the path $\psi_{D} \circ \gamma$, parameterized by capacity.

LEMMA 3.13 (Tameness invariance). For every monotone nondecreasing $\Upsilon:(0, \infty) \rightarrow(0,1]$ and every $r>1$, there is a monotone nondecreasing $\Upsilon^{*}:(0, \infty) \rightarrow(0,1]$ such that, for all $D \in \mathfrak{D}$ and $\gamma \in \mathcal{X}_{\Upsilon^{r}}(D), \gamma^{*} \in \mathcal{X}_{\Upsilon^{*}}(\mathbb{U})$.

Proof. Let $D \in \mathfrak{D}, \gamma \in \mathcal{X}_{\Upsilon}^{r}(D)$ and $0 \leq s_{1}^{\prime}<s_{2}^{\prime} \leq \infty$. Note that there exist $s_{1}$ and $s_{2}$ satisfying $s_{1}^{\prime} \leq s_{1} \leq s_{2} \leq s_{2}^{\prime}$ such that

$$
\operatorname{diam}\left(\gamma^{*}\left[s_{1}, s_{2}\right]\right) \geq \operatorname{diam}\left(\gamma^{*}\left[s_{1}^{\prime}, s_{2}^{\prime}\right]\right) / 4
$$

and

$$
\operatorname{dist}\left(0, \gamma^{*}\left[s_{1}, s_{2}\right]\right) \geq \operatorname{diam}\left(\gamma^{*}\left[s_{1}, s_{2}\right]\right)
$$

Since

$$
\operatorname{dist}\left(\gamma^{*}\left[0, s_{1}^{\prime}\right] \cup \partial \mathbb{U}, \gamma^{*}\left[s_{2}^{\prime}, \infty\right)\right) \geq \operatorname{dist}\left(\gamma^{*}\left[0, s_{1}\right] \cup \partial \mathbb{U}, \gamma^{*}\left[s_{2}, \infty\right)\right),
$$

it is sufficient to give a lower bound of the right-hand side of (3.27) in terms of $\epsilon:=\operatorname{diam}\left(\gamma^{*}\left[s_{1}, s_{2}\right]\right)$.

The Schwarz lemma gives $\psi_{D}^{\prime}(0) \leq 1 / \operatorname{rad}_{0}(D)$. Therefore, by the Koebe $1 / 4$ theorem (applied to the restriction of $\psi_{D}^{-1}$ to $\left.\epsilon \mathbb{U}\right)$ and (3.26), $\operatorname{dist}\left(0, \gamma\left[s_{1}, s_{2}\right]\right)>$ $c_{1} \operatorname{rad}_{0}(D)$, where $c_{1}=\epsilon / 4$. On the other hand, the harmonic measure in $\mathbb{U}$ from 0 of $\gamma^{*}\left[s_{1}, s_{2}\right]$ is at least $c_{2}$, where $c_{2}=c_{2}(\epsilon)>0$, so that the harmonic measure in $D$ from 0 of $\gamma\left[s_{1}, s_{2}\right]$ is at least $c_{2}$. Hence,

$$
\operatorname{diam} \gamma\left[s_{1}, s_{2}\right] \geq c_{3} \operatorname{rad}_{0}(D),
$$

where $c_{3}=c_{3}(\epsilon)$. 
Also set $\delta:=\operatorname{dist}\left(\gamma^{*}\left[0, s_{1}\right] \cup \partial \mathbb{U}, \gamma^{*}\left[s_{2}, \infty\right]\right)$. Since

$$
\operatorname{diam} \gamma^{*}\left[s_{2}, \infty\right] \geq \operatorname{dist}\left(0, \gamma^{*}\left[s_{1}, s_{2}\right]\right) \geq \epsilon,
$$

the extremal distance between $\gamma^{*}\left[0, s_{1}\right] \cup \partial \mathbb{U}$ and $\gamma^{*}\left[s_{2}, \infty\right]$ is at most $\phi_{1}(\delta, \epsilon)>0$, where $\phi_{1}$ is some function satisfying $\phi_{1}(\delta, \epsilon) \rightarrow 0$ as $\delta \downarrow 0$. By the conformal invariance of the extremal distance, this implies that the extremal distance between $\gamma\left[0, s_{1}\right] \cup \partial D$ and $\gamma\left[s_{2}, \infty\right]$ is at most $\phi_{1}(\delta, \epsilon)$. Because $\gamma$ is contained in the disk of radius $r \operatorname{rad}_{0}(D)$ about 0 , this implies that

$$
\operatorname{dist}\left(\gamma\left[0, s_{1}\right] \cup \partial D, \gamma\left[s_{2}, \infty\right]\right) \leq \phi_{2}(\delta, \epsilon) r \operatorname{rad}_{0}(D),
$$

where $\phi_{2} \rightarrow 0$ as $\delta \downarrow 0$. Because $\gamma \in \mathcal{X}_{\Upsilon}(D)$, (3.28) and this together imply

$$
\phi_{2}(\delta, \epsilon) r \geq \Upsilon\left(c_{3}(\epsilon)\right) \text {, }
$$

which gives a positive lower bound for $\delta=\operatorname{dist}\left(\gamma^{*}\left[0, s_{1}\right] \cup \partial \mathbb{U}, \gamma^{*}\left[s_{2}, \infty\right]\right)$ in terms of $\Upsilon, r$ and $\epsilon=\operatorname{diam}\left(\gamma^{*}\left[s_{1}, s_{2}\right]\right)$. This completes the proof.

LEMMA 3.14 (Convergence relations). Suppose $W^{n}, W$ are continuous functions from $[0, \infty)$ to $\partial \mathbb{U}$ such that $W^{n} \rightarrow W$ locally uniformly. Let $g_{t}^{n}, g_{t}$ be the corresponding solutions to Loewner's radial equation and set $f_{t}^{n}=\left(g_{t}^{n}\right)^{-1}$, $f_{t}=g_{t}^{-1}$. Then $f_{t}^{n} \rightarrow f_{t}$ locally uniformly on $[0, \infty) \times \mathbb{U}$. If there are continuous curves $\gamma^{n}:[0, \infty) \rightarrow \overline{\mathbb{U}}$ such that, for all $t \geq 0$, the image of $f_{t}^{n}$ is the component of 0 in $\mathbb{U} \backslash \gamma^{n}[0, t]$ and there is a $\gamma:[0, \infty) \rightarrow \overline{\mathbb{U}}$ such that $\gamma^{n} \rightarrow \gamma$ locally uniformly on $[0, \infty)$, then for all $t \geq 0$ the image of $f_{t}$ is the component of 0 in $\mathbb{U} \backslash \gamma[0, t]$.

PROOF. Since $g_{t}$ is obtained by flowing along a vector field depending on $W$, the inverse $f_{t}$ is obtained by flowing along the opposite field, with the time reversed. Hence, the first statement is an immediate consequence of the principle that solutions of ODE depend continuously on the parameters of the ODE. The second statement is an immediate consequence of the Carathéodory kernel theorem (see [35], Theorem 1.8).

PROOF OF THEOREM 3.9. Let $W^{n}$ denote the Loewner parameter of $\tilde{\gamma}^{n}$ and let $\hat{\mu}_{n}$ denote the law of the pair $\left(\tilde{\gamma}^{n}, W^{n}\right)$. By Theorem 3.7, we know that the law of $W^{n}$ tends weakly to the law of Brownian motion. Lemmas 3.10-3.13 show that the set of measures $\left\{\mu_{n}\right\}$ is tight with respect to the metric $\rho$. Consequently, the sequence $\hat{\mu}_{n}$ is also tight. Prokhorov's theorem (e.g., [9] and [37]) implies that there is a subsequence such that $\hat{\mu}_{n}$ converges weakly along the subsequence. Let $\hat{\mu}$ be any subsequential weak limit and let $(\tilde{\gamma}, W)$ be a sample from $\hat{\mu}$. The lemmas show that $\tilde{\gamma}$ is a.s. a simple path, and Theorem 3.7 shows that $W$ is Brownian motion (with time scaled). By the properties of weak convergence, we may couple the subsequence of pairs $\left(\tilde{\gamma}^{n}, W^{n}\right)$ and $(\tilde{\gamma}, W)$ so that a.s. $\rho\left(\tilde{\gamma}^{n}, \tilde{\gamma}\right) \rightarrow 0$ and $W^{n} \rightarrow W$ locally uniformly. 
Recall that the capacity is continuous with respect to the metric $\rho$; that is, if $\beta, \beta_{n}:[0,1] \rightarrow \overline{\mathbb{U}} \backslash\{0\}$ and $\rho\left(\beta_{n}, \beta\right) \rightarrow 0$, then the capacity of $\beta_{n}[0,1]$ tends to the capacity of $\beta[0,1]$. (In fact, it is enough that $\beta_{n}[0,1]$ tends to $\beta[0,1]$ in the Hausdorff metric.) Indeed, this follows immediately from Carathéodory's kernel theorem (see [35], Theorem 1.8) and the fact that the local uniform convergence of conformal maps implies the convergence of the derivatives (by Cauchy's formula for the derivative).

Since $\tilde{\gamma}$ is almost surely a simple path, the capacity of $\tilde{\gamma}$ increases strictly, and one can parameterize the path continuously by its capacity. We also parameterize the paths $\tilde{\gamma}^{n}$ by capacity. The next goal is to show that $\tilde{\gamma}^{n} \rightarrow \tilde{\gamma}$ locally uniformly on $[0, \infty)$. Since $\rho\left(\tilde{\gamma}, \tilde{\gamma}^{n}\right) \rightarrow 0$, there are strictly monotone continuous onto maps $\varepsilon_{n}:[0, \infty) \rightarrow[0, \infty)$ so that $\tilde{\gamma}^{n} \circ \varepsilon_{n} \rightarrow \tilde{\gamma}$ locally uniformly. If $t_{n} \in[0, \infty)$ and $t_{n} \rightarrow t \in[0, \infty)$, then it follows from the continuity of capacity with respect to $\rho$ that $\varepsilon_{n}\left(t_{n}\right) \rightarrow t$ [because if $s$ is a subsequential limit of $\varepsilon_{n}\left(t_{n}\right)$, then the capacity of $\tilde{\gamma}(s)$ must be $t$; i.e., $s=t$ ]. This implies that $\varepsilon_{n}$ converges to the identity map $t \mapsto t$, locally uniformly. By the continuity of $\tilde{\gamma}$, it follows that $\tilde{\gamma} \circ \varepsilon_{n}^{-1} \rightarrow \tilde{\gamma}$ locally uniformly. This gives $\tilde{\gamma}^{n} \rightarrow \tilde{\gamma}$ locally uniformly.

We can now finally apply Lemma 3.14 to show that $\tilde{\gamma}$ is the $\mathrm{SLE}_{2}$ path. As the law of the limit $\tilde{\gamma}$ does not depend on the subsequence, the theorem follows.

In the following proof of Theorem 1.1, the main technical point is that we do not make any smoothness assumptions on $\partial D$. If $\partial D$ is a simple closed path, the theorem follows easily from Theorem 3.9, because the suitably normalized conformal maps from $\mathbb{U}$ to the discrete approximations of $D$ converge uniformly to the conformal map onto $D$.

ProOF OF THEOREM 1.1. Let $D_{\delta}$ be the component of 0 in the complement of all the closed square faces of the grid $\delta \mathbb{Z}^{2}$ intersecting $\partial D$. Let $\gamma_{\delta}$ be the time reversal of the loop-erased random walk from 0 to $\partial D_{\delta}$ and let $\beta$ be the radial $\mathrm{SLE}_{2}$ path in $\bar{U}$. Let $\phi_{\delta}: \mathbb{U} \rightarrow D_{\delta}$ be the conformal map satisfying $\phi_{\delta}(0)=0$ and $\phi_{\delta}^{\prime}(0)>0$ and let $\phi: \mathbb{U} \rightarrow D$ be the conformal map satisfying $\phi(0)=0, \phi^{\prime}(0)>0$. Theorem 3.9 tells us that we may couple $\beta$ with each of the paths $\gamma_{\delta}$ such that $\rho\left(\phi_{\delta}^{-1} \circ \gamma_{\delta}, \beta\right) \rightarrow 0$ in probability as $\delta \downarrow 0$. Moreover, the proof shows that if we use the capacity parameterization for both, then, in probability,

$$
\sup \left\{\left|\phi_{\delta}^{-1} \circ \gamma_{\delta}(t)-\beta(t)\right|: t \geq 0\right\} \rightarrow 0 .
$$

(There is no problem with convergence in a neighborhood of $t=\infty$, because we know that the weak limit of $\phi_{\delta}^{-1} \circ \gamma_{\delta}$ with respect to $\rho$ is a simple path tending to 0 as $t \rightarrow \infty$.)

The Carathéodory kernel theorem (see [35], Theorem 1.8) implies that $\phi_{\delta} \rightarrow \phi$ uniformly on compact subsets of $\mathbb{U}$ as $\delta \searrow 0$. Consequently, the above gives

$$
\forall t_{0}>0, \quad \sup \left\{\left|\gamma_{\delta}(t)-\phi \circ \beta(t)\right|: t \geq t_{0}\right\} \rightarrow 0,
$$


in probability. Let $\epsilon>0$ be small. Then, by Lemma 3.11, there is an $\epsilon^{\prime}>0$ such that for every $D^{\prime} \in \mathfrak{D}$ the probability that a simple random walk from 0 gets to distance $\operatorname{rad}_{0}\left(D^{\prime}\right) /\left(2 \epsilon^{\prime}\right)$ before hitting $\partial D^{\prime}$ is less than $\epsilon / 2$. Let $A$ be the connected component of 0 in the set of points in $D \cap\left(\operatorname{rad}_{0}(D) / \epsilon^{\prime}\right) \mathbb{U}$ having distance at least $\epsilon \epsilon^{\prime} \operatorname{rad}_{0}(D)$ from $\partial D$. By considering the first point where the random walk generating $\gamma_{\delta}$ exits $A$, it follows that, with probability at least $1-\epsilon$, the diameter of $\gamma_{\delta}[0, \infty] \backslash A$ is at most $\epsilon \operatorname{rad}_{0}(D)+\delta$. Now note that there is a compact $A^{\prime} \subset \mathbb{U}$ such that $\phi_{\delta}^{-1}(A) \subset A^{\prime}$ for all sufficiently small $\delta$, since $\phi_{\delta} \rightarrow \phi$ uniformly on compacts. Therefore, there is some $t_{1}>0$ such that $\gamma_{\delta}\left[0, t_{1}\right] \cap A=\varnothing$ a.s. for all sufficiently small $\delta>0$. In particular,

$$
\mathbf{P}\left[\operatorname{diam} \gamma_{\delta}\left[0, t_{1}\right]>\epsilon \operatorname{rad}_{0}(D)+\delta\right]<\epsilon .
$$

If we take $t_{2} \in\left(0, t_{1}\right)$, then taking $\delta \searrow 0$ in (3.29) implies

$$
\mathbf{P}\left[\operatorname{diam} \phi \circ \beta\left[t_{2}, t_{1}\right]>2 \epsilon \operatorname{rad}_{0}(D)\right]<\epsilon .
$$

Since this holds for every $t_{2}$, it follows that

$$
\mathbf{P}\left[\operatorname{diam} \phi \circ \beta\left(0, t_{1}\right]>2 \epsilon \operatorname{rad}_{0}(D)\right]<\epsilon .
$$

Using this with (3.30) and choosing $t_{0}=t_{1}$ in (3.29) gives

$$
\mathbf{P}\left[\sup \left\{\left|\gamma_{\delta}(t)-\phi \circ \beta(t)\right|: t>0\right\}<3 \epsilon \operatorname{rad}_{0}(D)\right] \rightarrow 1 .
$$

Since this holds for every $\epsilon>0$, the theorem follows.

\section{The UST Peano curve.}

4.1. Setup. The UST Peano curve is obtained as the interface between the UST and the dual UST. The setup which corresponds to chordal $\mathrm{SLE}_{8}$ is where there is symmetry between the UST and the dual UST. Loosely speaking, the UST is the uniform spanning tree on the grid inside a domain $D$ but with an entire arc $\alpha \subset \partial D$ on the boundary identified (wired) as a single vertex, and the dual UST also has an $\operatorname{arc} \beta \subset \partial D$ on the boundary which is identified. The arcs $\alpha$ and $\beta$ are essentially complementary arcs. See Figure 5 , where $D$ is approximately a rectangle. As mentioned in Section 1, it was conjectured in [36] that, for an analogous setup, the interface defined for the critical random cluster models with $q \in(0,4]$ converges to $\mathrm{SLE}_{\kappa}$, where $\kappa=\kappa(q) \in[4,8)$.

A combinatorial framework is necessary in order to be more precise. There are several different possible setups that would work, and the following is somewhat arbitrary.

If a tree $T$ lies in the grid $\mathbb{Z}^{2}$, then its dual tree $T^{\dagger}$ will lie in the dual grid $(\mathbb{Z}+1 / 2)^{2}$, and the Peano path $\gamma$ will lie in the graph $G$ whose vertices are $(1 / 4+\mathbb{Z} / 2)^{2}$ and where $v, u$ neighbor iff $|v-u|=1 / 2$. We have three kinds of vertices: elements of $\mathbb{Z}^{2}$ are the primal vertices, elements of $(1 / 2+\mathbb{Z})^{2}$ are the dual 


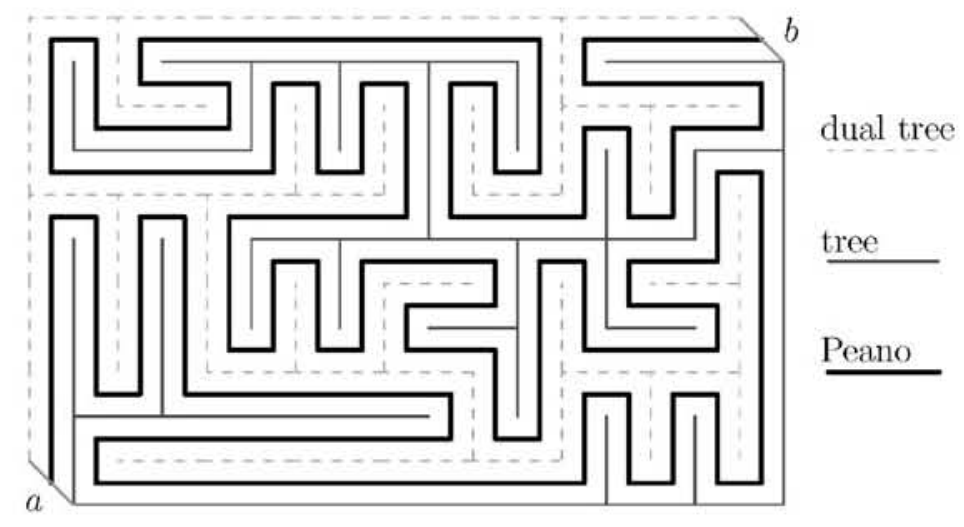

FIG. 5. The tree, dual tree and Peano UST path $\gamma$.

vertices and elements of $(1 / 4+\mathbb{Z} / 2)^{2}$ are the Peano vertices. If $w \neq v$ are vertices of any kind, not necessarily the same, we say that they are adjacent if the distance between them is as small as it can be for distinct vertices of these particular kinds. In other words, if they are of the same kind, this means that they are neighbors, if $v \in(1 / 4+\mathbb{Z} / 2)^{2}$ and $w \in \mathbb{Z}^{2} \cup(1 / 2+\mathbb{Z})^{2}$, this means $\|v-w\|_{\infty}=1 / 4$, while if $v \in \mathbb{Z}^{2}$ and $w \in(1 / 2+\mathbb{Z})^{2}$, this means $\|v-w\|_{\infty}=1 / 2$.

Since there is no added complication, we consider a more general case where $\alpha$ and $\beta$ are trees, rather than arcs. Let $\alpha$ be some finite tree in the primal grid $\mathbb{Z}^{2}$ and let $\beta$ be a finite tree in the dual grid $(1 / 2+\mathbb{Z})^{2}$. Suppose that no edge of $\alpha$ intersects an edge of $\beta$. Further suppose that there are two Peano vertices $a, b \in(1 / 4+\mathbb{Z} / 2)^{2}$ such that $a$ is adjacent to both a primal vertex $\alpha_{a} \in \alpha$ and a dual vertex $\beta_{a} \in \beta$, and $b$ is adjacent to both a primal vertex $\alpha_{b} \in \alpha$ and a dual vertex $\beta_{b} \in \beta$. See Figure 6 . Note that the line segment $\left[\alpha_{a}, \beta_{a}\right]$ has $a$ as its midpoint, and the line segment $\left[\alpha_{b}, \beta_{b}\right]$ has $b$ as its midpoint. Let $D=D(\alpha, \beta, a, b)$ be the (unique) bounded

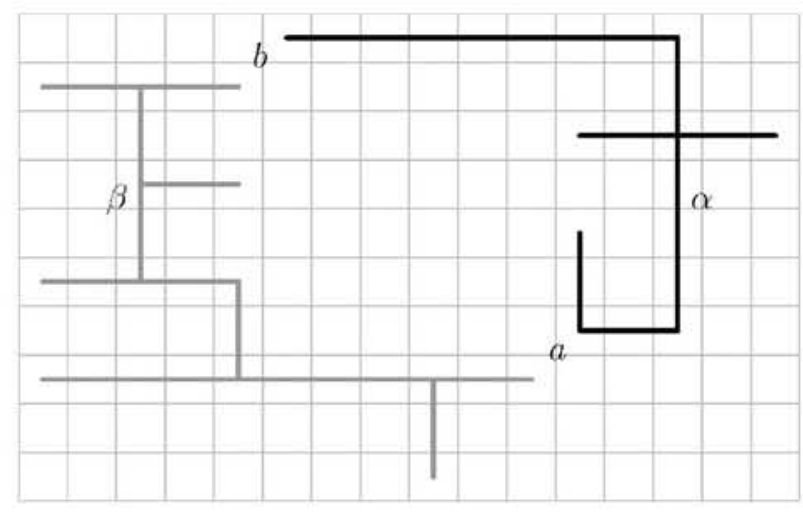

FIG. 6. The boundary data and the Peano grid. 
connected component of $\mathbb{C} \backslash\left(\alpha \cup\left[\alpha_{b}, \beta_{b}\right] \cup \beta \cup\left[\beta_{a}, \alpha_{a}\right]\right)$. Let $V_{P}=V_{P}(D)$ denote the collection of all Peano vertices in $\bar{D}$, and, as before, $V(D)$ denotes the collection of all primal vertices in $D$. Let $\ell=\ell(D)$ denote the cardinality of $V_{P} \backslash\{a, b\}$. By switching the role of $a$ and $b$, if necessary, assume that $D$ lies to the immediate right of the oriented segment $\left[\alpha_{a}, \beta_{a}\right]$. Let $\mathfrak{D}^{*}$ denote the collection of all domains obtained in this way.

Let $H=H(D)$ denote the subgraph of $\mathbb{Z}^{2}$ whose vertices are the vertices of $\alpha$ and $V(D)$ and whose edges are those edges on this set of vertices which do not intersect $\beta$. Since $\beta$ is a tree, $H$ is connected.

Since $H$ is connected, there is at least one spanning tree $T$ of $H$ which contains $\alpha$. If we replace $\alpha$ by $T$ and apply the dual argument, it follows that there is also a tree $T^{\dagger}$ in the dual grid $(1 / 2+\mathbb{Z})^{2}$, which is disjoint from $T$, contains $\beta$ and whose vertices are the dual vertices in $\beta$ and the dual vertices in $D$. In fact, $T^{\dagger}$ contains every dual edge lying in $\bar{D} \backslash T$.

We now need to give an orientation to the Peano grid $G$. Every edge in $G$ is either on the boundary of a square face of $G$ centered on a primal vertex or on the boundary of a square face of $G$ centered on a dual vertex, and these two possibilities are exclusive. We orient the edges of $G$ by specifying that the square faces of $G$ containing a primal vertex are oriented clockwise, while those containing a dual vertex are oriented counterclockwise. When we want to emphasize the orientation of the edges, we write $G_{\rightarrow}$ instead of $G$. Note that the edges of $G$ contained in a horizontal or vertical line all get the same direction in $G_{\rightarrow}$, and consecutive parallel lines get opposite orientations. For this reason, $G_{\rightarrow}$ is often called the Manhattan lattice.

Let $\gamma=\gamma(T)$ denote the set of all edges of $G_{\rightarrow}$ which do not intersect $T \cup T^{\dagger}$ and which have at least one endpoint in $D$. Let $v \in V_{P} \backslash\{a, b\}$ be some Peano vertex in $D$. Note that there are precisely two oriented edges of $G_{\rightarrow}$ with initial point $v$, say $e_{1}$ and $e_{2}$, where one of these, say $e_{1}$, intersects an edge $f_{1}$ of the primal grid $\mathbb{Z}^{2}$, and the other intersects an edge $f_{2}$ of the dual grid $(1 / 2+\mathbb{Z})^{2}$. Note also that $f_{1} \cap f_{2} \neq \varnothing$. It therefore follows that exactly one of the edges $f_{1}, f_{2}$ is in $T \cup T^{\dagger}$. Consequently, exactly one of the edges $e_{1}, e_{2}$ is in $\gamma$. This shows that $\gamma$ has out-degree 1 at every $v \in V_{P} \backslash\{a, b\}$. An entirely similar argument shows that $\gamma$ has in-degree 1 at every such $v$. In particular, this shows that $\gamma$ does not contain the entire boundary of a square face of $G$ that does not contain a primal or dual vertex. If $\gamma$ had a cycle, the cycle therefore would have to surround some primal or dual vertex. But as $T$ and $T^{\dagger}$ are connected and disjoint from $\gamma$, this is impossible. It therefore follows that $\gamma$ is an oriented simple path (i.e., self-avoiding path), and the endpoints of $\gamma$ are $a$ and $b$. Since we are assuming that $D$ lies to the right of $\left[\alpha_{a}, \beta_{a}\right]$, the initial point of $\gamma$ is $a$ and the terminal point is $b$.

Conversely, suppose that $\gamma^{*}=\left(\gamma_{0}^{*}, \ldots, \gamma_{\ell+1}^{*}\right)$ is any oriented simple path in $G_{\rightarrow}$, respecting the orientation of $G_{\rightarrow}$, from $a$ to $b$, whose vertices are $V_{P}$. For $n \in\{0, \ldots, \ell+1\}$, let $v_{n}$ be the (unique) primal vertex adjacent to $\gamma_{n}^{*}$ and 
let $v_{n}^{\dagger}$ be the dual vertex adjacent to $\gamma_{n}^{*}$. Note that $v_{n}$ and $v_{n+1}$ are either the same vertex or adjacent vertices when $n=\{0, \ldots, \ell\}$. Let $\alpha_{n}=\alpha_{n}\left(\gamma^{*}\right)$ denote the union of $\alpha$ with the collection of all edges $\left[v_{k}, v_{k+1}\right]$ for $k<n$ such that $v_{k} \neq v_{k+1}$ and, similarly, let $\beta=\beta_{n}\left(\gamma^{*}\right)$ denote the union of $\beta$ with the collection of all dual edges $\left[v_{k}^{\dagger}, v_{k+1}^{\dagger}\right]$ for $k<n$ such that $v_{k}^{\dagger} \neq v_{k+1}^{\dagger}$. Then $T\left(\gamma^{*}\right):=\alpha_{\ell+1}\left(\gamma^{*}\right)$ and $T^{\dagger}\left(\gamma^{*}\right):=\beta_{\ell+1}\left(\gamma^{*}\right)$ are obviously connected, and there are no edges in $T\left(\gamma^{*}\right)$ intersecting edges in $T^{\dagger}\left(\gamma^{*}\right)$. Now, $T\left(\gamma^{*}\right)$ cannot contain a cycle, for such a cycle would have to separate $T^{\dagger}\left(\gamma^{*}\right)$. Hence, $T\left(\gamma^{*}\right)$ is a spanning tree of $H$ containing $\alpha$. It is also clear that $\gamma^{*}=\gamma\left(T\left(\gamma^{*}\right)\right)$. That is, $T \mapsto \gamma(T)$ is a bijection between the set of spanning trees of $H$ containing $\alpha$ and the set of oriented paths in $G \rightarrow \cap \bar{D}$ from $a$ to $b$ containing $V_{P}$. Hence, when $T$ is the UST on $H$ conditioned to contain $\alpha, \gamma$ is uniformly distributed among such Peano paths; it is the UST Peano path associated with $(\alpha, \beta, a, b)$.

Let $\left(a=w_{0}, w_{1}, \ldots, w_{\ell+1}=b\right)$ be the order of the vertices in the UST Peano path $\gamma$. For $n \in\{0,1, \ldots, \ell\}$, let $\gamma[0, n]$ denote the initial arc of $\gamma$ from $w_{0}$ to $w_{n}$. Since $\gamma$ is uniformly distributed among simple oriented paths in $G_{\rightarrow}$ from $a$ to $b$ which contain $V_{P}$, we immediately get the following Markov property.

LEMMA 4.1 (Markovian property). Fix any $n \in\{1,2, \ldots, \ell\}$. Conditioned on $\gamma[0, n]$, the distribution of $(\gamma \backslash \gamma[0, n]) \cup\left\{w_{n}\right\}$ is the same as that of the UST Peano curve associated with $\left(\alpha_{n}(\gamma), \beta_{n}(\gamma), w_{n}, b\right)$.

This lemma will play the same role in the proof as Lemma 3.2 in the case of the LERW. We will also use the convergence of certain discrete harmonic functions toward their continuous counterparts. To facilitate this, we have to set the combinatorial notation for the discrete Dirichlet-Neumann problem.

Let $H$ be a finite nonempty connected subgraph of $\mathbb{Z}^{2}$ with vertices $V_{H}$ and let $E_{\partial}$ denote the set of oriented edges in $\mathbb{Z}^{2}$ whose initial endpoint is in $V_{H}$, but whose unoriented version is not in $H$. Suppose $E_{\partial}=E_{0} \cup E_{1} \cup E_{2}$ is a disjoint union, where $E_{0} \cup E_{1} \neq \varnothing$. Suppose also that $\hat{h}: V_{H} \rightarrow[0,1]$ is some function. For $v \in V_{H}$, set $\Delta_{H, E_{0}, E_{1}, E_{2}} \hat{h}(v):=\sum d \hat{h}[v, u]$, where the sum is over all neighbors $u$ of $v$ in $\mathbb{Z}^{2}$, and $d \hat{h}[v, u]:=\hat{h}(u)-\hat{h}(v)$ when $[v, u] \notin E_{\partial}$, $d \hat{h}[v, u]:=0-\hat{h}(v)$ when $[v, u] \in E_{0}, d \hat{h}[v, u]:=1-\hat{h}(v)$ when $[v, u] \in E_{1}$ and $d \hat{h}[v, u]:=0$ when $[v, u] \in E_{2}$. Note that there is a unique $\hat{h}: V_{H} \rightarrow[0,1]$ such that $\Delta_{H, E_{0}, E_{1}, E_{2}} \hat{h}(v)=0$ in $V_{H}: \hat{h}(v)$ is the probability that a simple random walk on $H \cup E_{0} \cup E_{1}$ started from $v$ will use an edge in $E_{1}$ before using an edge of $E_{0}$. This $\hat{h}$ will be called the $\Delta_{H, E_{0}, E_{1}, E_{2}}$-harmonic function.

Proposition 4.2 (Dirichlet-Neumann approximation). For every $\epsilon>0$, there is an $r_{0}=r_{0}(\epsilon)$ such that the following holds. Let $D \subset \mathbb{C}$ be a simply connected domain satisfying $\operatorname{rad}_{0}(D) \geq r_{0}$. Let $A_{0}, A_{1} \subset \partial \mathbb{U}$ be two disjoint arcs, each of length at least $\epsilon$, and set $A_{2}:=\partial U \backslash\left(A_{0} \cup A_{1}\right)$. Let $\eta \subset D$ be a simple 
closed path which surrounds 0 , such that each point of $\eta$ is within distance 5 from $\partial D$. Suppose that $A_{0}^{\prime}, A_{1}^{\prime} \subset \eta$ are two disjoint arcs, $A_{2}^{\prime}:=\eta \backslash\left(A_{0}^{\prime} \cup A_{1}^{\prime}\right)$ and the triple $\left(A_{0}^{\prime}, A_{1}^{\prime}, A_{2}^{\prime}\right)$ corresponds to $\left(A_{0}, A_{1}, A_{2}\right)$ under $\psi_{D}$, in the sense that for each $j=0,1,2$ and each $p \in A_{j}^{\prime}$ there is a continuous path $\sigma:[0,1) \rightarrow D$ satisfying $\operatorname{diam} \sigma[0,1) \leq 5, \sigma(0)=p$, and $\lim _{s \uparrow 1} \psi_{D} \circ \sigma(s)$ exists and is in $A_{j}$.

Let $H$ be the component of 0 in the set of edges of $\mathbb{Z}^{2}$ that do not intersect $\eta$. For $j=0,1,2$, let $E_{j}$ denote the set of oriented edges $[v, u]$ intersecting $\eta$, where $v$ is in $H$, and the first point of intersection from the direction of $v$ is in $A_{j}^{\prime}$. Let

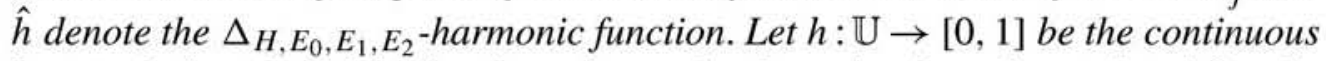
harmonic function which has boundary value 0 on $A_{0}, 1$ on $A_{1}$, and satisfies the Neumann boundary condition on $A_{2}$. Then $|\hat{h}(0)-h(0)|<\epsilon$.

The proof will be given in Section 5.4.

4.2. Driving process convergence. Let $\alpha, \beta, a, b$ and $D=D(\alpha, \beta, a, b)$ be as above and suppose now that $0 \in D$. As before, let $\ell$ denote the number of Peano vertices in $D$ and let $\gamma=(\gamma(0), \ldots, \gamma(\ell+1))$ be the UST Peano path from $a$ to $b$ in $G_{\rightarrow} \cap \bar{D}$. For each $n \leq \ell$, there are two domains that are naturally associated to $\gamma[0, n]$. The first one (as in Lemma 4.1) is $\tilde{D}_{n}:=D\left(\alpha_{n}, \beta_{n}, \gamma(n), b\right)$ (see Figure 7). But $\tilde{D}_{n}$ is not so useful if we want to make estimates using Loewner's equation. We therefore also define $D_{n}:=D \backslash \gamma[0, n]$. Let $\phi_{0}=\phi: D \rightarrow \mathbb{H}$ be the conformal map which takes $D$ to $\mathbb{H}$, takes $b$ to $\infty$, takes $a$ to 0 and satisfies $|\phi(0)|=1$. Let $\phi_{n}: D_{n} \rightarrow \mathbb{H}$ be the conformal maps satisfying $\phi_{n}(z)-\phi_{0}(z) \rightarrow 0$ as $z \rightarrow b$ within $D_{n}$. Define $W_{n}:=\phi_{n}(\gamma(n)) \in \mathbb{R}$. Also let $t_{n}$ denote the capacity from $\infty$ in $\mathbb{H}$ of $\phi_{0} \circ \gamma[0, n]$, so that $\phi_{n} \circ \phi_{0}^{-1}(z)=z+2 t_{n} / z+o(1 / z)$ when $z \rightarrow \infty$ in $\mathbb{H}$.

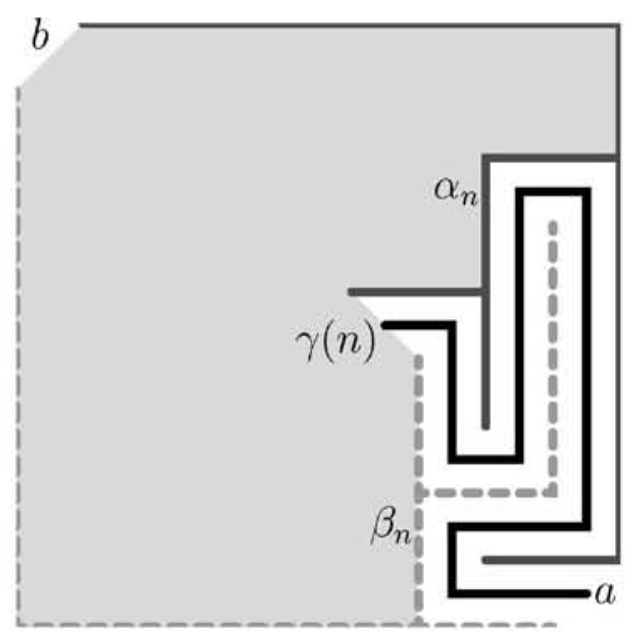

FIG. 7. The domain $\tilde{D}_{n}$ is shaded. 
We now prove the analog of Proposition 3.4 for the UST Peano curve. Let $\mathfrak{H}_{D}(z, A)$ denote the continuous harmonic measure of $A$ from $z$ in the domain $D \backslash A$.

Proposition 4.3 (The key estimate). For every sufficiently small $\delta, \epsilon>0$, there is some $r_{0}=r_{0}(\delta, \epsilon)$ such that the following holds. Let $\gamma, D_{n}, \phi_{n}, W_{n}$ and $t_{n}$ be as above, let $k \in \mathbb{N}$ and let $m$ be the first $n \geq k$ such that $\left|W_{n}-W_{k}\right| \geq \delta$ or $t_{n}-t_{k} \geq \delta^{2}$. Then

$$
\mathbf{E}\left[W_{m}-W_{k} \mid D_{k}\right]=O\left(\delta^{3}\right)
$$

and

$$
\mathbf{E}\left[\left(W_{m}-W_{k}\right)^{2} \mid D_{k}\right]=8 \mathbf{E}\left[t_{m}-t_{k} \mid D_{k}\right]+O\left(\delta^{3}\right),
$$

provided that $\operatorname{rad}_{0}\left(D_{k}\right) \geq r_{0}$ and $\mathfrak{H}_{D_{k}}\left(0, \alpha_{k}(\gamma)\right) \in[\epsilon, 1-\epsilon]$.

ProOF. Assume first $k=0$. Let $v_{0} \in V(D)$ be some vertex such that $\left|\phi\left(v_{0}\right)\right| \leq 2$ and $\operatorname{Im} \phi\left(v_{0}\right) \geq 1 / 2$, say. [As we have seen in Section 3.2, there is such a $v_{0}$ when $\operatorname{rad}_{0}(D)$ is large.] If $Q=\left[q, q^{\prime}\right]$ is a line segment where $q \in D$ is a dual vertex and $q^{\prime} \in \alpha$ is the midpoint of a dual edge containing $q$, then let $\phi^{*}(Q) \in \mathbb{R}_{+}$ denote the limit of $\phi(z)$ as $z$ tends to $\partial D$ along $Q$ (which always exists by [35], Proposition 2.14). Fix such a $Q_{0}$ satisfying $U:=\phi^{*}\left(Q_{0}\right) \in[1 / 2,2]$; there clearly is such $Q_{0}$ when $r_{0}$ is large, because the harmonic measure from 0 of any square of the dual grid adjacent to the boundary of $D$ is small. Let $\eta \subset D$ be the set of points within distance $1 / 10$ from $\partial D$. Then $\eta$ is a simple closed path. Consider it as oriented counterclockwise around the bounded domain of $\mathbb{C} \backslash \eta$. Let $p_{0}$ be the point of $\eta$ closest to $a, p_{1}$ the point in $\eta \cap Q_{0}$ and $p_{2}$ the point of $\eta$ closest to $b$. Let $A_{0}^{\prime}$ be the positively oriented subarc of $\eta$ from $p_{0}$ and $p_{1}, A_{1}^{\prime}$ the positively oriented arc from $p_{1}$ to $p_{2}$ and $A_{2}^{\prime}$ the positively oriented arc from $p_{2}$ to $p_{0}$.

Let $\mathcal{A}$ be the event that the path in the tree $T(\gamma)=\alpha_{\ell+1}(\gamma)$ from $v_{0}$ to $\alpha$ hits $A_{1}^{\prime}$. We will now estimate both sides of the identity

$$
\mathbf{P}[\mathcal{A}]=\mathbf{E}\left[\mathbf{P}\left[\mathcal{A} \mid D_{m}\right]\right]
$$

using Proposition 4.2. By Wilson's algorithm, $\mathbf{P}[\mathcal{A}]$ is the probability that a simple random walk on the graph $H(D)$ started at $v_{0}$ stopped on hitting $\alpha$ will cross $A_{1}^{\prime}$. This is exactly $\hat{h}\left(v_{0}\right)$, where the function $\hat{h}$ is as defined in Proposition 4.2. Set

$$
h(z):=\frac{1}{\pi} \cot ^{-1}\left(\frac{1-|z|}{2 \operatorname{Im} \sqrt{z}}\right)=\frac{1}{\pi} \cot ^{-1}\left(\frac{1-r}{2 \sqrt{r} \sin (\theta / 2)}\right),
$$

where $z=r e^{i \theta}$ and we take the value of $\cot ^{-1}$ between 0 and $\pi$. Note that $h$ is harmonic in $\mathbb{H}$, is equal to 0 on $(0,1)$, is equal to 1 on $(1, \infty)$, and $\partial_{y} h=0$ on $(-\infty, 0)$. (Of course, we found the map $h$ satisfying these boundary conditions by reflecting the domain along the negative real axis, mapping this larger domain to $\mathbb{H}$ 
with $z \mapsto \sqrt{z}$ and then using a conformal map from $\mathbb{H}$ to $\mathbb{U}$ to calculate the hitting probabilities.) Consequently, Proposition 4.2 shows that if $r_{0}$ is sufficiently large, then

$$
\mathbf{P}[\mathcal{A}]=h\left(\phi\left(v_{0}\right) / U\right)+O\left(\delta^{3}\right) .
$$

Set $V_{j}:=\phi_{j}\left(v_{0}\right)$ and $U_{j}:=\phi_{j} \circ \phi_{0}^{-1}(U)$. By the chordal version of Loewner's equation and the definition of $m$, we have

$$
V_{m}=V_{0}+\frac{2 t_{m}}{V_{0}}+O\left(\delta^{3}\right), \quad U_{m}=U_{0}+\frac{2 t_{m}}{U_{0}}+O\left(\delta^{3}\right) .
$$

Note also that $\operatorname{rad}_{0}\left(D_{m}\right)>\operatorname{rad}_{0}(D) / 2, v_{0} \in D_{m}$ and $U_{m} \in[1 / 4,4]$ provided that $\delta$ is small enough.

We now employ a similar argument to estimate $\mathbf{P}\left[\mathcal{A} \mid D_{m}\right]$. Recall that $\tilde{D}_{n}=$ $D\left(\alpha_{n}, \beta_{n}, \gamma(n), b\right)$. Assume that $Q_{0}$ intersects $\tilde{D}_{n}$, which will be the case if $U_{n}>1 / 4$, say. Let $\eta_{n}$ be the set of points in $\tilde{D}_{n}$ at distance $1 / 10$ from $\partial \tilde{D}_{n}$. Again, $\eta_{n}$ is a simple closed path, and we write $\eta_{n}=A_{0}^{\prime}(n) \cup A_{1}^{\prime}(n) \cup A_{2}^{\prime}(n)$, where $A_{0}^{\prime}(n)$ is the arc of $\eta_{n}$ from the closest point to $\gamma(n)$ to the point of intersection of $Q_{0}$ with $\eta_{n}, A_{1}^{\prime}(n)$ is the arc of $\eta_{n}$ from the point in $Q_{0} \cap \eta_{n}$ to the point of $\eta_{n}$ closest to $b$ and $A_{2}^{\prime}(n)$ is the remaining part of $\eta_{n}$. By Lemma 4.1, P $\left[\mathcal{A} \mid D_{n}\right]$ is the same as the quantity $\hat{h}_{n}\left(v_{0}\right)$, where $\hat{h}_{n}$ is the function $\hat{h}$ defined in Proposition 4.2, but with $A_{0}^{\prime}(n), A_{1}^{\prime}(n), A_{2}^{\prime}(n)$ and $\eta_{n}$ replacing $A_{0}^{\prime}, A_{1}^{\prime}, A_{2}^{\prime}$ and $\eta$ and $D_{n}$ replacing $D$. (It is $D_{n}$ replacing $D$, not $\tilde{D}_{n}$. The conditions of Proposition 4.2 hold for either of these, but the conformal map we consider is defined on $D_{n}$.) Proposition 4.2 therefore gives

$$
\mathbf{P}\left[\mathcal{A} \mid D_{m}\right]=h\left(\frac{V_{m}-W_{m}}{U_{m}-W_{m}}\right)+O\left(\delta^{3}\right) .
$$

Write $f(U, V, W):=h((V-W) /(U-W))$. We Taylor-expand the right-hand side in (4.6) to second order in $W_{m}$ and to first order in $V_{m}-V_{0}$ and $U_{m}-U_{0}$. Together with (4.3)-(4.5) this gives

$$
\begin{aligned}
0 & =\mathbf{E}\left[\mathbf{P}\left[\mathcal{A} \mid D_{m}\right]\right]-\mathbf{P}[\mathcal{A}] \\
& =\frac{1}{2} \partial_{W}^{2} f \mathbf{E}\left[W_{m}^{2}\right]+\partial_{W} f \mathbf{E}\left[W_{m}\right]+\partial_{V} f \frac{2 \mathbf{E}\left[t_{m}\right]}{V_{0}}+\partial_{U} f \frac{2 \mathbf{E}\left[t_{m}\right]}{U_{0}}+O\left(\delta^{3}\right) .
\end{aligned}
$$

Here, the derivatives of $f$ are evaluated at $\left(V_{0}, W_{0}, U_{0}\right)$. (Note that $V$ is complex valued, and we interpret $\partial_{V} f$ as an $\mathbb{R}$-linear map from $\mathbb{C}$ to $\mathbb{R}$.) If we plug in $V_{0}=i+O\left(\delta^{3}\right)$ and $U_{0}=1+O\left(\delta^{3}\right)$ [as we have seen in Section 3.2, one can certainly find $v_{0}$ and $u_{0}$ satisfying $\phi\left(v_{0}\right)=i+O\left(\delta^{3}\right)$ and $\phi\left(u_{0}\right)=1+O\left(\delta^{3}\right)$ if $r_{0}$ is large], then after some tedious but straightforward computations the above equality simplifies to

$$
\mathbf{E}\left[W_{m}^{2}\right]+2 \mathbf{E}\left[W_{m}\right]-8 \mathbf{E}\left[t_{m}\right]=O\left(\delta^{3}\right),
$$


while $V_{0}=2 i+O\left(\delta^{3}\right)$ and $U_{0}=1+O\left(\delta^{3}\right)$ give

$$
\left.3 \mathbf{E}\left[W_{m}^{2}\right]+8 \mathbf{E}\left[W_{m}\right]-24 \mathbf{E}\left[t_{m}\right]=O \delta^{3}\right) .
$$

Combining these two relations implies (4.1) and (4.2) in the case $k=0$. For $k>0$, the proof is basically the same; the only essential difference is that one must use $\eta_{k}$ in place of $\eta$.

THEOREM 4.4 (Driving process convergence). For every positive $\epsilon_{1}, \epsilon_{2}, \epsilon_{3}$ and $\bar{t}$, there is some positive $r_{1}=r_{1}\left(\epsilon_{1}, \epsilon_{2}, \epsilon_{3}, \bar{t}\right)$ such that the following holds. Let $D=D(\alpha, \beta, a, b) \in \mathfrak{D}^{*}$ satisfy $\operatorname{rad}_{0}(D)>r_{1}$ and $\mathfrak{H}_{D}(0, \alpha) \in\left[\epsilon_{1}, 1-\epsilon_{1}\right]$. Let $\gamma$ be the corresponding UST Peano path, let $\phi: D \rightarrow \mathbb{H}$ denote the conformal map which takes a to $0, b$ to $\infty$ and satisfies $|\phi(0)|=1$, let $\hat{\gamma}:=\phi \circ \gamma$, parameterized according to capacity from $\infty$, and let $W(t)$ denote the Loewner driving process for $\hat{\gamma}$. Then there is a coupling of standard Brownian motion $B:[0, \bar{t}] \rightarrow \mathbb{R}$ and $W$ such that

$$
\mathbf{P}\left[\sup \{|W(t)-B(8 t)|: t \in[0, \bar{t}]\}>\epsilon_{2}\right]<\epsilon_{3} .
$$

PROOF. The proof is almost identical to the proof of Theorem 3.7, where we used Skorohod's embedding, but one has to be a little careful because it may happen that 0 is "swallowed" before time $\bar{t}$.

Let us first assume that $\phi(0)$ is close to $i$, say $|\phi(0)-i|<1 / 100$, and that $\bar{t}$ is small enough so that

$$
\bar{t} \leq 1 / 100 \quad \text { and } \quad \mathbf{P}[B[0, \bar{t}] \subset[-1 / 10,1 / 10]]>1-\epsilon_{3} / 3,
$$

where $B$ is standard Brownian motion. Take $\delta=\delta\left(\epsilon_{1}, \epsilon_{2}, \epsilon_{3}\right)>0$ small and $\epsilon=1 / 10$. Define $r_{0}(\epsilon, \delta)$ as in Proposition 4.3. Let $k \in \mathbb{N}$ be the first integer where $\operatorname{rad}_{0}\left(D_{k}\right) \leq r_{0}$ or $\mathfrak{H}_{D_{k}}\left(0, \alpha_{k}\right) \notin[\epsilon, 1-\epsilon]$ and define $\bar{t}_{0}:=\min \left\{\bar{t}, t_{k}\right\}$, where $t_{n}$ is as in the proposition. Exactly as in the proof of Theorem 3.7, Proposition 4.3 implies that we may couple $W$ with a Brownian motion $B$ in such a way that

$$
\mathbf{P}\left[\sup \left\{|W(t)-B(8 t)|: t \in\left[0, \bar{t}_{0}\right]\right\}>\epsilon_{2} / 3\right]<\epsilon_{3} / 3,
$$

if $\operatorname{rad}_{0}(D) \geq r_{1}$ and $r_{1}$ is large enough. By our assumptions regarding $\bar{t}$, we have with high probability that, for all $t \in\left[0, \bar{t}_{0}\right], W(t) \in[-1 / 5,1 / 5]$. If we choose $r_{1}$ large enough, this guarantees that $\mathbf{P}\left[\bar{t}_{0} \neq \bar{t}\right]<\epsilon_{3} / 3$ and proves the theorem when (4.7) is satisfied and $\phi(0)$ is close to $i$.

Consider now a general $\bar{t}<\infty$. Let $\bar{t}_{1}>0$ be some constant satisfying (4.7) and let $z_{0}:=\phi^{-1}\left(2 i \bar{t}^{2} / \bar{t}_{1}^{2}\right)$. From the Koebe distortion theorem, it follows that there is a constant $c=c\left(\bar{t}, \epsilon_{1}\right)$ such that $\operatorname{rad}_{z_{0}}(D) \geq c \operatorname{rad}_{0}(D)$. (See, e.g., Theorem 1.3 and Corollary 1.4 in [35].) Consequently, by choosing $r_{1}$ appropriately larger, we may invoke the above argument with the basepoint moved from 0 to a vertex near $z_{0}$ and with a smaller $\epsilon_{2}$. Rescaling now completes the proof of the theorem. 
4.3. Uniform continuity. In order to prove convergence with respect to a stronger topology, tightness will be needed, and we therefore derive in this section some regularity estimates for UST Peano curves with respect to the capacity parameterization. Some results from [38] will be used.

Let $D \subset \mathbb{C}$ be a simply connected domain containing 0 , whose boundary is a $C^{1}$-simple closed path. Let $a$ and $b$ be two distinct points on $\partial D$. In this section, we consider for large $R$ the UST Peano curve from a point near $R a$ to a point near $R b$ on a grid approximation of $R D$. One reason not to consider arbitrary domains is that we need to partially adapt to the framework of [38] in order to quote results from there. Also, it is natural (since the UST Peano curve is asymptotically space filling) to impose regularity conditions on $\partial D$ in order to get uniform regularity estimates for the UST Peano curve.

Let $\alpha_{D}$ and $\beta_{D}$ be, respectively, the clockwise and anticlockwise arcs of $\partial D$ from $b$ to $a$. Given $R$ large, let $D^{R}=D\left(\alpha^{R}, \beta^{R}, a^{R}, b^{R}\right) \in \mathfrak{D}^{*}$ be an approximation of $\left(R D, R \alpha_{D}, R \beta_{D}\right)$ in the following sense. Fix some sufficiently large constant $C>0$; for example, $C=10$ would do. We require $\alpha^{R}$ to be a simple path in $\mathbb{Z}^{2}$ satisfying $\rho\left(\alpha^{R}, R \alpha_{D}\right) \leq C$ and require $\beta^{R}$ to be a simple path in the dual grid $(1 / 2+\mathbb{Z})^{2}$ satisfying $\rho\left(\beta^{R}, R \beta_{D}\right) \leq C$. We also require that $\beta^{R} \cap \alpha^{R}=\varnothing$, of course, and that each of $a^{R}, b^{R}$ is a Peano vertex adjacent to an endpoint of $\alpha^{R}$ and an endpoint of $\beta^{R}$.

Let $\gamma=\gamma^{R}$ be the UST Peano path in $D^{R}$. Let $\phi: D \rightarrow \mathbb{H}$ be the conformal homeomorphism satisfying $\phi(a)=0, \phi(b)=\infty$ and $|\phi(0)|=1$. Let $\phi_{R}: D^{R} \rightarrow \mathbb{H}$ be the conformal homeomorphism satisfying $\left|\phi_{R}(0)\right|=1$, taking $a^{R}$ to 0 and $b^{R}$ to $\infty$. Then $\lim _{R \rightarrow \infty} R^{-1} \phi_{R}^{-1}(z)=\phi^{-1}(z)$ uniformly in $\mathbb{H}$. (This follows, e.g., from Corollary 2.4 in [35].) Let $\hat{\gamma}:=\phi_{R} \circ \gamma$, parameterized according to capacity from $\infty$. Let $g_{t}: \mathbb{H} \backslash \hat{\gamma}[0, t] \rightarrow \mathbb{H}$ be the conformal map with the usual normalization $g_{t}(z)-z \rightarrow 0$ when $|z| \rightarrow \infty$.

Proposition 4.5 (Uniform continuity estimate). For every $\epsilon>0$ and $\bar{t}>0$, there are some positive $R_{0}=R_{0}(D, \bar{t}, \epsilon)$ and $\delta=\delta(D, \bar{t}, \epsilon)$ such that, for all $R>R_{0}$,

$$
\mathbf{P}\left[\sup \left\{\left|\hat{\gamma}\left(t_{2}\right)-\hat{\gamma}\left(t_{1}\right)\right|: t_{1}, t_{2} \in[0, \bar{t}],\left|t_{2}-t_{1}\right| \leq \delta\right\}>\epsilon\right]<\epsilon .
$$

We first prove a slightly modified version of this proposition.

LEMMA 4.6. For $0<t_{1}<t_{2}<\infty$, let $Y\left(t_{1}, t_{2}\right):=\operatorname{diam}\left(g_{t_{1}} \circ \hat{\gamma}\left[t_{1}, t_{2}\right]\right)$. For every $\epsilon>0$, there is a $\delta=\delta(D, \epsilon)>0$ and an $R_{0}=R_{0}(D, \epsilon)>0$ such that, for all $R \geq R_{0}$,

$$
\mathbf{P}\left[\sup \left\{\left|\hat{\gamma}\left(t_{2}\right)-\hat{\gamma}\left(t_{1}\right)\right|: 0 \leq t_{1} \leq t_{2} \leq \tau, Y\left(t_{1}, t_{2}\right) \leq \delta\right\} \geq \epsilon\right]<\epsilon,
$$

where $\tau:=\inf \left\{t \geq 0:|\hat{\gamma}(t)|=\epsilon^{-1}\right\}$. 
The proof will use Theorems 10.7 and 11.1(ii) of [38]. As explained there, the proofs of these theorems are now easier, because we have established the conformal invariance of the UST; see Corollary 1.2.

Proof of Lemma 4.6. Let $\delta_{R}$ be a positive function of $R$ such that $\lim _{R \rightarrow \infty} \delta_{R}=0$. It suffices to show that (4.8) holds for all sufficiently large $R$ with $\delta_{R}$ in place of $\delta$. Let $Z$ denote the semicircle $2 \epsilon^{-1} \partial \mathbb{U} \cap \mathbb{H}$, say. For $R$ large, let $t_{1}$ and $t_{2}$ be such that $\left|\hat{\gamma}\left(t_{2}\right)-\hat{\gamma}\left(t_{1}\right)\right|$ is maximal subject to the constraints $0 \leq t_{1} \leq t_{2} \leq \tau$ and $Y\left(t_{1}, t_{2}\right) \leq \delta_{R}$. Note that $\min _{t<\tau} \operatorname{dist}\left(g_{t}(Z), g_{t} \circ \hat{\gamma}[t, \tau]\right)$ is bounded from below, as $\hat{\gamma}[0, \tau] \subset \epsilon^{-1} \overline{\mathbb{U}}$. [Considering the harmonic measure from a point near $\infty$, one deduces that the diameter of $g_{t}((1 / 2) Z)$ is bounded below. The extremal distance between $g_{t}(Z)$ and $g_{t}((1 / 2) Z)$ is the same as the extremal distance between $Z$ and $(1 / 2) Z$. This shows that $\operatorname{dist}\left(g_{t}(Z), g_{t} \circ \hat{\gamma}[t, \tau]\right) \geq$ $\operatorname{dist}\left(g_{t}(Z), g_{t}((1 / 2) Z)\right)$ is bounded from below.] Since $Y\left(t_{1}, t_{2}\right) \leq \delta_{R} \rightarrow 0$ as $R \rightarrow \infty$, the extremal length of the collection of simple arcs in $\mathbb{H} \backslash g_{t_{1}} \circ \hat{\gamma}\left[t_{1}, t_{2}\right]$ which separate $g_{t_{1}} \circ \hat{\gamma}\left[t_{1}, t_{2}\right]$ from $g_{t_{1}}(Z)$ goes to 0 . By the conformal invariance of the extremal length, it follows that the extremal length of the collection of simple arcs in $\mathbb{H} \backslash \hat{\gamma}\left[t_{1}, t_{2}\right]$ which separate $\hat{\gamma}\left[t_{1}, t_{2}\right]$ from $Z$ in $\mathbb{H} \backslash \hat{\gamma}\left[0, t_{1}\right]$ tends to 0 as well. In particular, the shortest such arc for the Euclidean metric, say $\eta$, satisfies $\lim _{R \rightarrow \infty}$ length $(\eta)=0$.

We are going to study separately the three cases where $\eta$ is close to the origin, close to the real line but not to the origin and not close to the real line. In each case, we will see that the existence of such an $\eta$ is very unlikely. Let $\mathcal{A}$ be the event $\left|\hat{\gamma}\left(t_{1}\right)-\hat{\gamma}\left(t_{2}\right)\right| \geq \epsilon$. For $s>0$, let $X_{0}(s)$ be the event dist $(0, \eta)<s$ and let $X_{1}(s)$ be the event $\operatorname{dist}(\mathbb{R}, \eta)<s$. We will prove

$$
\begin{aligned}
\forall s_{1}>0, \exists R_{0}>0, \forall R>R_{0}, \quad \mathbf{P}\left[\mathcal{A} \backslash \mathcal{X}_{1}\left(s_{1}\right)\right]<\epsilon, \\
\forall s_{0}>0, \exists s_{1}>0, \exists R_{0}>0, \forall R>R_{0}, \\
\quad \mathbf{P}\left[\mathcal{A} \cap \mathcal{X}_{1}\left(s_{1}\right) \backslash \mathcal{X}_{0}\left(s_{0}\right)\right]<\epsilon, \\
\exists s_{0}>0, \exists R_{0}>0, \forall R>R_{0}, \quad \mathbf{P}\left[\mathcal{A} \cap \mathcal{X}_{0}\left(s_{0}\right)\right]<\epsilon .
\end{aligned}
$$

Using these statements, the proof of the lemma is completed by choosing $s_{0}$ according to (4.11), then choosing $s_{1}$ according to (4.10) and, finally, choosing $R_{0}$ according to (4.9), (4.10) and (4.11).

We start with (4.9). Fix some $s_{1}>0$ and assume that $\mathcal{A} \backslash \mathcal{X}_{1}\left(s_{1}\right)$ holds. We also assume that $\epsilon<s_{1}$. There is no loss of generality in that assumption, since $\mathcal{A}$ is monotone decreasing in $\epsilon$. Since $\lim _{R \rightarrow \infty}$ length $(\eta)=0$, for large $R$ the two endpoints of $\eta$ must be in $\hat{\gamma}\left[0, t_{1}\right]$. Because $\hat{\gamma}$ tends to $\infty$ with $t$, it is clear that $\hat{\gamma}\left[t_{2}, \infty\right) \cap \eta \neq \varnothing$. In fact, the crossing number of $\hat{\gamma}\left[t_{2}, \infty\right)$ and $\eta$ must be \pm 1 , since $\hat{\gamma}$ and $\eta$ are simple curves. Consider the concentric annulus $A$ whose inner circle is the smallest circle surrounding $\eta$ and whose outer circle has radius $\epsilon / 4$. Let $\mathcal{B}$ denote the open disk bounded by the outer circle of $A$ and note that $\mathscr{B} \subset \mathbb{H}$, by our assumption $\epsilon<s_{1}$. On the event $\mathcal{A}$, there is a $t^{*} \in\left[t_{1}, t_{2}\right]$ such 
that the distance from $\hat{\gamma}\left(t^{*}\right)$ to $\eta$ is at least $\epsilon / 2$. In particular, $\hat{\gamma}\left(t^{*}\right) \notin \mathscr{B}$. Now, $\eta$ separates $\hat{\gamma}\left(t^{*}\right)$ from $\infty$ in $\mathbb{H} \backslash \hat{\gamma}\left[0, t_{1}\right]$. Therefore, if $\mathcal{A} \backslash X_{1}(s)$ holds, then $\hat{\gamma}\left[0, t_{1}\right] \cup \eta$ separates $\hat{\gamma}\left(t^{*}\right)$ from $\infty$. Since $\hat{\gamma}$ is a simple path, this implies that the arc of $\hat{\gamma}\left[0, t_{1}\right]$ between the two points $\bar{\eta} \cap \hat{\gamma}\left[0, t_{1}\right]$ does not stay in $\mathscr{B}$. Hence, $\hat{\gamma}[0, \infty) \cap \mathscr{B}$ has three distinct connected components, say $\hat{\gamma}_{1}, \hat{\gamma}_{2}, \hat{\gamma}_{3}$, each of which intersects the inner circle of $A$ such that $\hat{\gamma}_{1}, \hat{\gamma}_{3} \subset \hat{\gamma}\left[0, t^{*}\right]$ and $\hat{\gamma}_{2} \subset \hat{\gamma}\left[t^{*}, \infty\right)$ and $\hat{\gamma}_{2}$ separates $\hat{\gamma}_{1}$ from $\hat{\gamma}_{3}$ within $\mathscr{B}$. See Figure 8 .

Note that adjacent to one side of $\phi_{R}^{-1}\left(\hat{\gamma}_{2}\right)$ lies $T$, the UST, and $T^{\dagger}$, the dual UST, is adjacent to the other side. Both are connected, and they do not intersect $\phi_{R}^{-1}(\hat{\gamma})$. It follows that there are paths $\chi_{1} \subset T$ and $\chi_{2} \subset T^{\dagger}$ with endpoints in $\phi_{R}^{-1}(\partial \mathscr{B})$, each of which intersects the inner boundary of $\phi_{R}^{-1}(A)$. But the diameter of the inner boundary of $R^{-1} \phi_{R}^{-1}(A)$ goes to 0 as $R \rightarrow \infty$, and the distance between the two boundary components of $R^{-1} \phi_{R}^{-1}(A)$ does not. Hence, by [38], Theorem 10.7, the probability that such a configuration appears somewhere goes to 0 with $R$. (Although the result from [38] refers to the UST in the whole plane, the proof is local, and since we are bounded away from the boundary, the result is applicable here.) This proves (4.9).

Now fix $s_{0}>0$ and let $s_{1}>0$ be much smaller. Assume that $\mathcal{A} \cap \mathcal{X}_{1}\left(s_{1}\right) \backslash$ $\chi_{0}\left(s_{0}\right)$ holds, $\epsilon<s_{1}$ and $R$ is large. Also assume that $\eta$ is closer to $[0, \infty)$ than to $(-\infty, 0]$. Note that $\eta$ is then bounded away from $(-\infty, 0]$. Let $A$ be defined as above and let $\mathcal{B}$ be the intersection of $\mathbb{H}$ with the disk bounded by the outer boundary component of $A$. We now need to consider two distinct possibilities. Either both endpoints of $\eta$ are on $\hat{\gamma}\left[0, t_{1}\right]$, and then the configuration is topologically as in the argument for (4.9), or one endpoint of $\eta$ is on $[0, \infty)$. But it is easy to see that in either case there is a simple path in $T^{\dagger}$ which intersects $\phi_{R}^{-1}(\eta)$ whose endpoints are in $\phi_{R}^{-1}(\partial \mathscr{B})$, by an argument very similar to the one given above. Now [38], Theorem 11.1(ii), shows that these events have small probabilities if $s_{1}$ is small. The case where $\eta$ is closer to $(-\infty, 0]$ is treated similarly, with the roles of the tree and the dual tree switched. Thus, (4.10) is established.

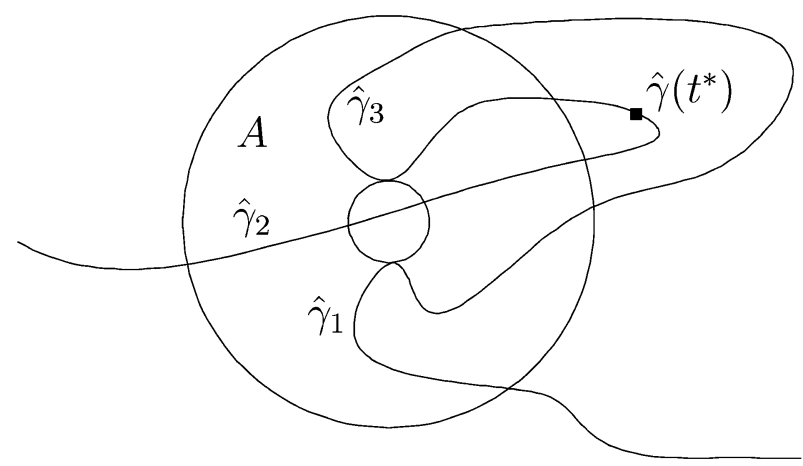

FIG. 8. The paths $\hat{\gamma}_{1}, \hat{\gamma}_{2}$ and $\hat{\gamma}_{3}$ and the annulus $A$. 
To prove (4.11), let $s>0$ and let $v \in \mathbb{Z}^{2}$ be a vertex closest to $\phi_{R}^{-1}(i s)$. Let $v^{\dagger} \in(\mathbb{Z}+1 / 2)^{2}$ be a dual vertex adjacent to $v$. Let $\chi$ be the simple path from $v$ to $\alpha^{R}$ in $T$ and let $\chi^{\dagger}$ be the simple path from $v^{\dagger}$ to $\beta^{R}$ in $T^{\dagger}$. We may sample $\chi$ by running a simple random walk from $v$ on $H\left(D^{R}\right)$ stopped on hitting $\alpha^{R}$ and loop-erasing it. It therefore follows by Proposition 4.2 that if $s$ is sufficiently small, then the diameter of $\phi_{R}(\chi)$ is smaller than $\epsilon / 10$ with probability at least $1-\epsilon / 10$. Moreover, there is some $s_{0}>0$ such that for all sufficiently large $R$ with probability at least $1-\epsilon / 10$ the distance from $\phi_{R}(\chi)$ to 0 is at least $s_{0}$, and the same two estimates will hold for $\chi^{\dagger}$. Let $D^{\prime}$ be the domain bounded by $\left[v, v^{\dagger}\right] \cup \chi \cup \chi^{\dagger} \cup \partial D^{R}$ which has the initial point $a^{R}$ of $\gamma$ on its boundary. Note that $\gamma$ crosses the boundary of $D^{\prime}$ exactly once, through the segment $\left[v, v^{\dagger}\right]$. In particular, if $\operatorname{diam}\left(\phi_{R}\left(D^{\prime}\right)\right)<\epsilon$ and $\mathcal{A}$ holds, then $\eta$ is not contained in $\phi_{R}\left(D^{\prime}\right)$. This proves (4.11) and completes the proof of the lemma.

ProOf OF Proposition 4.5. Theorem 4.4 implies that we may find some $r>0$ such that $\mathbf{P}[\sup \{|W(t)|: t \in[0, \bar{t}]\} \geq r]<\epsilon / 4$ for all sufficiently large $R$. Let $\epsilon^{\prime}:=\min \left\{\epsilon, r^{-1}\right\}$ and let $\delta^{\prime}$ denote the $\delta$ obtained by using Lemma 4.6 with $\epsilon^{\prime}$ in place of $\epsilon$. Since Brownian motion is a.s. continuous, Theorem 4.4 implies that there is some $\delta>0$ such that if $R$ is large enough we have

$$
\mathbf{P}\left[\sup \left\{\left|W\left(t_{1}\right)-W\left(t_{2}\right)\right|: t_{1}, t_{2} \in[0, \bar{t}],\left|t_{1}-t_{2}\right|<\delta\right\} \geq \delta^{\prime}\right]<\epsilon / 4 .
$$

Lemma 2.1 applied to the path $t \mapsto g_{t_{1}} \circ \gamma\left(t-t_{1}\right)-W\left(t_{1}\right)$ now implies

$$
\mathbf{P}\left[\sup \left\{Y\left(t_{1}, t_{2}\right): 0 \leq t_{1} \leq t_{2} \leq \bar{t},\left|t_{1}-t_{2}\right|<\delta\right\} \geq C\left(\delta^{1 / 2}+\delta^{\prime}\right)\right]<\epsilon / 4 .
$$

Now the proof is completed by using Lemma 4.6.

4.4. Consequences. In this section we gather some consequences, starting with the following two theorems.

THEOREM 4.7 (Chordal SLE 8 traces a path). Let $\tilde{g}_{t}$ denote the chordal $\mathrm{SLE}_{8}$ process driven by $B(8 t)$, where $B(t)$ is standard Brownian motion. Then, a.s. for every $t>0$, the map $\tilde{g}_{t}^{-1}$ extends continuously to $\overline{\mathbb{H}}$ and $\tilde{\gamma}(t):=\tilde{g}_{t}^{-1}(B(8 t))$ is a.s. continuous. Moreover, a.s. $\tilde{g}_{t}^{-1}(\mathbb{H})$ is the unbounded component of $\mathbb{H} \backslash \tilde{\gamma}[0, t]$ for every $t \geq 0$.

THEOREM 4.8 (Peano path convergence). Let $D \subset \mathbb{C}$ be a domain containing 0 such that $\partial D$ is a $C^{1}$-smooth simple closed path. Let $\partial D=\alpha_{D} \cup \beta_{D}$ be a partition of the boundary of $D$ into two nontrivial complementary arcs. For $R>0$, let $\left(D^{R}, \alpha^{R}, \beta^{R}\right)$ be an approximation of $(R D, R \alpha, R \beta)$, as described in Section 4.3. Let $\gamma=\gamma^{R}$ denote the UST Peano curve in $D^{R}$ with the corresponding boundary conditions. Let $\phi_{R}: D^{R} \rightarrow \mathbb{H}$ denote the conformal map which takes the initial point of $\gamma$ to 0 , the terminal point to $\infty$ and satisfies $\left|\phi_{R}(0)\right|=1$. Let $\hat{\gamma}:=\phi_{R} \circ \gamma$, parameterized by capacity from $\infty$. Then the law of $\hat{\gamma}$ tends weakly to the law of $\tilde{\gamma}$ from Theorem 4.7. 
Here, we think of $\hat{\gamma}$ and $\tilde{\gamma}$ as elements of the space of continuous maps from $[0, \infty)$ to $\overline{\mathbb{H}}$, with the topology of locally uniform convergence.

A consequence of the theorem is that $R^{-1} \gamma$ is close to $R^{-1} \phi_{R}^{-1} \circ \tilde{\gamma}$. That is, we may approximate the UST Peano path $\gamma$ by the image of chordal SLE 8 in $D^{R}$.

The analog of Theorem 4.7 was proven in [36] for all $\kappa \neq 8$, but the particular case $\kappa=8$ could not be handled there. It is fortunate that the convergence of the UST Peano path to $\mathrm{SLE}_{8}$ settles this problem. By Remark 7.5 in [36], it follows that with the notation of Theorem 4.7 for $\kappa \geq 8$ we have $\tilde{g}_{t}^{-1}(\mathbb{H})=\mathbb{H} \backslash \tilde{\gamma}[0, t]$ for every $t \geq 0$ a.s.

Proof OF THeOREMS 4.7 AND 4.8. Let $W(t)=W_{R}(t)$ denote the chordal Loewner driving process for $\hat{\gamma}$. Fix a sequence $R_{n} \rightarrow \infty$. First, note that the family of laws of $\hat{\gamma}$ is tight, because of Proposition 4.5 and the Arzela-Ascoli theorem (see, for instance, [17], Theorem 2.4.10). Also, Theorem 4.4 implies that the law of $W$ converges weakly to the law of $B(8 t)$. Hence, there is a subsequence of $R_{n}$ such that the law of the pair $(\hat{\gamma}, W)$ converges weakly to some probability measure $\mu$. Let $\left(\gamma^{*}, W^{*}\right)$ be random with law $\mu$. Then we may identify $W^{*}(t)$ with $B(8 t)$. By the chordal analog of Lemma 3.14, which is valid with the same proof, it follows that, for all $t>0, \tilde{g}_{t}^{-1}(\mathbb{H})$ is the unbounded component of $\mathbb{H} \backslash \gamma^{*}[0, t]$. Since $\gamma^{*}$ is continuous, elementary properties of conformal maps imply that $\tilde{g}_{t}^{-1}$ extends continuously to $\overline{\mathbb{H}}$ (e.g., Theorem 2.1 in [35]). It is easy to verify that, a.s. for every $t>0, \tilde{g}_{t}^{-1}(B(8 t))=\gamma^{*}(t)$, using the fact that $\gamma^{*}\left[t, t^{\prime}\right]$ is contained in a small neighborhood of $\gamma^{*}(t)$ when $t^{\prime}-t>0$ is small. This proves Theorem 4.7. Because the law of the limit path $\gamma^{*}$ does not depend on the subsequence, the original sequence converges, and so Theorem 4.8 is proved as well.

We now list some easy consequences of Theorems 4.7 and 4.8 .

COROLlaRY 4.9 (Radial $\mathrm{SLE}_{8}$ traces a path). Let $\tilde{g}_{t}$ denote a radial $\mathrm{SLE}_{8}$ process driven by $W(t)=\exp (i B(8 t))$, where $B$ is standard Brownian motion. Then, almost surely, for every $t>0$, the map $\tilde{g}_{t}^{-1}$ extends continuously to $\overline{\mathbb{U}}$. Moreover, $\tilde{g}_{t}^{-1}(W(t))$ is almost surely continuous.

ProOF. This follows readily from Theorem 4.7 and the absolute continuity relation between radial and chordal $\mathrm{SLE}_{8}$ derived in [28], Proposition 4.2.

Proof of Theorem 1.3. Define $G_{\delta}=\delta D^{1 / \delta}$, where $D^{1 / \delta}$ is defined as in Theorem 4.8. Consider the situation of Theorem 4.8. As previously remarked, it follows from [35], Corollary 2.4, that $\lim _{R \rightarrow \infty} R^{-1} \phi_{R}^{-1}(z)=\phi^{-1}(z)$ uniformly in $\mathbb{H}$. Consequently, Theorem 4.8 shows that, for all $\bar{t}>0$, the UST Peano curve scaling limit up to capacity $\bar{t}$ from $b$ is equal to $\phi^{-1} \circ \tilde{\gamma}$ up to time $\bar{t}$. It therefore suffices to prove that for all $\epsilon>0$ there is an $\epsilon^{\prime}>0$ such that for all sufficiently 
large $R$ with probability at least $1-\epsilon$ the part of $\gamma$ after the first time it hits the $\epsilon^{\prime}$-neighborhood of $b$ stays within the $\epsilon$-neighborhood of $b$. This is easily proved by the same argument used to prove (4.11) applied to the reversal of the UST Peano path, which is also a UST Peano path.

COROLlaRY 4.10 (Path reversal). The law of the chordal $\mathrm{SLE}_{8}$ curve is invariant under simultaneously reversing time and inverting in the unit circle, up to a monotone increasing time change. More precisely, if $\tilde{\gamma}$ is the chordal $\mathrm{SLE}_{8}$ curve from 0 to $\infty$ defined in Theorem 4.7, then a time change of $(-1 / \tilde{\gamma}(1 / t), t \geq 0)$ has the same law as $\tilde{\gamma}$.

PROOF. This follows immediately from the fact that the reversed UST Peano curve is also a UST Peano curve.

5. Random walk estimates. The goal of this section is to prove the remaining random walk estimates and thereby complete the proofs of the theorems. Basically, we show that, under certain boundary conditions, discrete harmonic functions converge to continuous harmonic functions satisfying corresponding boundary conditions, as the mesh of the grid goes to 0 . This general principle is not new, of course (see, e.g., [6]), but it seems that the precise statements which are needed here do not appear in the literature. In particular, our results make no smoothness assumptions on the boundary. It should perhaps be noted that some of the following proofs (and most likely the results, too) are special to two dimensions.

5.1. Preliminary lemmas. We now state some lemmas on discrete harmonic functions, which will be helpful in the proofs of Proposition 2.2, Lemma 3.5 and Proposition 4.2.

For $\delta>0$, define the discrete derivatives

$$
\begin{aligned}
& \partial_{x}^{\delta} f(v):=\delta^{-1}(f(v+\delta)-f(v)), \\
& \partial_{y}^{\delta} f(v):=\delta^{-1}(f(v+i \delta)-f(v)) .
\end{aligned}
$$

Let $\mathfrak{D}_{\delta}:=\{\delta D: D \in \mathfrak{D}\}$, that is, domains adapted to the grid $\delta \mathbb{Z}^{2}$. Similarly, for $D_{\delta}=\delta D \in \mathfrak{D}_{\delta}$, define $V^{\delta}\left(D_{\delta}\right):=D_{\delta} \cap \delta \mathbb{Z}^{2}=\delta V(D)$ and $V_{\partial}^{\delta}\left(D_{\delta}\right):=\delta V_{\partial}(D)$.

LEMMA 5.1 (Discrete derivative estimate). There is a constant $C>0$ such that, for every $D \in \mathfrak{D}$ and every bounded function $h: V(D) \cup V_{\partial}(D) \rightarrow \mathbb{R}$ that is harmonic in $V(D)$,

$$
\partial_{x}^{1} h(0) \leq C \operatorname{rad}_{0}(D)^{-1}\|h\|_{\infty}, \quad \partial_{y}^{1} h(0) \leq C \operatorname{rad}_{0}(D)^{-1}\|h\|_{\infty} .
$$

This lemma is proved using the Green's functions in [25], Theorem 1.7.1; see also [16], Lemma 7.1, for a proof of the analogous statement in the triangular lattice using the maximum principle. In Section 6, we rewrite and adapt the proof from [25] to more general walks on planar lattices. One can also rather easily prove the lemma using coupling. 
LeMmA 5.2. For all $\epsilon>0$ and $k \in \mathbb{N}$, there exists $a c=c_{k}(\epsilon)>0$ such that the following always holds. Let $\delta \in\left(0, c^{-1}\right)$ and let $D \in \mathfrak{D}_{\delta}$ satisfy $\operatorname{rad}_{0}(D) \geq 1 / 2$. Let $\partial_{a_{1}}^{\delta}, \ldots, \partial_{a_{k}}^{\delta} \in\left\{\partial_{x}^{\delta}, \partial_{y}^{\delta}\right\}$. Let $h: V^{\delta}(D) \cup V_{\partial}^{\delta}(D) \rightarrow[0, \infty)$ be nonnegative and harmonic in $V^{\delta}(D)$. If $v \in V^{\delta}(D)$ satisfies $\left|\psi_{D}(v)\right| \leq 1-\epsilon$, then

$$
\left|\partial_{a_{1}}^{\delta} \partial_{a_{2}}^{\delta} \cdots \partial_{a_{k}}^{\delta} h(v)\right| \leq \operatorname{ch}(0) \text {. }
$$

Note that the case $k=0$, which is included, is a kind of Harnack inequality.

It is easy to give quantitative estimates for $c_{k}(\epsilon)$, but they will not be needed here. Only $k \leq 3$ will be used in the rest of the paper.

In the proof of the lemma, the following simple conformal geometry consequences of the Koebe distortion theorem (see [35], Theorem 1.3) will be needed. Let $D, \epsilon$ and $v$ be as in the statement of the lemma. First, note that $1 / 4 \leq$ $\operatorname{rad}_{0}(D) \psi_{D}^{\prime}(0) \leq 1$ follows from the Koebe $1 / 4$ theorem and the Schwarz lemma, respectively. Let $\ell=\ell(\epsilon)$ be large and set $z_{j}:=j \psi_{D}(v) / \ell$ and $w_{j}:=\psi_{D}^{-1}\left(z_{j}\right)$, $j=0,1, \ldots, \ell$. The Koebe distortion theorem gives upper and lower bounds for $\operatorname{rad}_{0}(D)\left|\psi_{D}^{\prime}\right|$ on the preimage of the line segment $\left[0, z_{\ell}\right]$. This implies that there is a constant $c_{1}=c_{1}(\epsilon)>0$ such that $\operatorname{rad}_{w_{j}}(D) \geq c_{1} \operatorname{rad}_{0}(D)$ and that if $\ell=\ell(\epsilon)$ is large, then, $\left|w_{j}-w_{j-1}\right| \leq c_{1} \operatorname{rad}_{0}(D) / 20, j=1, \ldots, \ell$. In particular, if $v_{j}$ is the vertex in $V^{\delta}(D)$ closest to $w_{j}$, then, provided that $\delta$ is sufficiently small, $\left|v_{j}-v_{j-1}\right| \leq \operatorname{rad}_{v_{j-1}}(D) / 10$.

Proof OF Lemma 5.2. We start with $k=0$. Suppose first that $|v| \leq$ $\operatorname{rad}_{0}(D) / 10$. Let $W \subset V^{\delta}(D)$ be the set of vertices $w$ satisfying $h(w) \geq h(v)$. Then $W$ contains a path from $v$ to $\partial D$. But the probability $p$ that the path traced by a simple random walk from 0 before exiting $D$ separates $v$ from $\partial D$ is bounded away from 0 . On that event, the simple random walk hits $W$ before exiting $D$. Consequently, $h(0) \geq p h(v)$, as needed. For arbitrary $v \in V^{\delta}(D)$ satisfying $\left|\psi_{D}(v)\right| \leq 1-\epsilon$, as we have noted, the Koebe distortion theorem implies that there is an $\ell=\ell(\epsilon)$ depending only on $\epsilon$ and a sequence $0=v_{0}, v_{1}, \ldots, v_{\ell}=v$ in $V^{\delta}(D)$ with $\ell \leq \ell(\epsilon)$ such that $\left|v_{j}-v_{j-1}\right| \leq \operatorname{rad}_{v_{j}}(D) / 10$ for each $j=1, \ldots, \ell$. Consequently, iterating the above result gives $h(0) \geq p^{\ell} h(v)$ and proves the case $k=0$.

Using the above, we know that $h(w) \leq c^{\prime} h(0)$ on the set of vertices $w \in V^{\delta}(D)$ such that $|w-v| \leq \operatorname{rad}_{v}(D) / 10$, where $c^{\prime}=c^{\prime}(\epsilon)$ is some constant depending only on $\epsilon$. Consequently, the case $k=1$ now follows from Lemma 5.1 applied with $v$ translated to 0 .

For $k>1$, the proof is by induction. By the above, we may assume $v=0$. Let $M$ be the maximum of $\left|\partial_{a_{k}}^{\delta} h(w)\right| / h(0)$ on the set $V$ of vertices $w \in V^{\delta}(D)$ satisfying $|w| \leq \operatorname{rad}_{0}(D) / 10$. The above shows that $M$ is bounded by a universal constant. Since $\partial_{a_{k}}^{\delta} h$ is discrete harmonic on $V$, the proof is completed by applying the inductive hypotheses to the function $\partial_{a_{k}}^{\delta} h(w)+M h(0)$. 
LEMMA 5.3 (Continuous harmonic approximation). For every $\epsilon>0$, there is some $r_{0}=r_{0}(\epsilon)>0$ such that the following holds. If $D \in \mathfrak{D}$ satisfies $\operatorname{rad}_{0}(D) \geq r_{0}$ and $h: V(D) \cup V_{\partial}(D) \rightarrow[0, \infty)$ is discrete harmonic in $V(D)$, then there exists a harmonic function $h^{*}: D \rightarrow[0, \infty)$ such that

$$
\left|h^{*}(v)-h(v)\right| \leq \epsilon h(0)
$$

holds for every vertex $v \in V(D)$ satisfying $\left|\psi_{D}(v)\right|<1-\epsilon$.

Proof. Suppose that the lemma is not true. Then there exists $\epsilon>0$ and a sequence of pairs $\left(D_{n}, h_{n}\right)$, where $D_{n} \in \mathfrak{D}$ satisfies $\operatorname{rad}_{0}\left(D_{n}\right) \geq n$ and $h_{n}>0$ is discrete harmonic in $V\left(D_{n}\right)$, satisfies $h_{n}(0)=1$, but (5.3) fails for every harmonic function $\hat{h}$.

Set $\delta=\delta_{n}:=1 / \operatorname{rad}_{0}\left(D_{n}\right)$. Our objective is to apply compactness to show that the maps $h_{n} \circ \psi_{D}^{-1}$ converge locally uniformly in $U$ as $n \rightarrow \infty$ along some subsequence to some harmonic $\hat{h}$, so that (5.3) does hold for some $n$. We put $h^{n}(v):=h_{n}\left(v / \delta_{n}\right)$.

First, standard compactness properties of conformal maps say that one can take a subsequence such that the maps $\delta_{n} \psi_{D_{n}}^{-1}$ converge locally uniformly in $\mathbb{U}$ to some conformal map, say $\phi$. (This follows, e.g., from the Arzela-Ascoli theorem, together with [35], Corollary 1.4, with $z=0$ and part two of [35], Theorem 1.3.) If $K \subset \mathbb{U}$ is compact, then Lemma 5.2 shows that there is a constant $C>0$ such that, for all sufficiently large $n$ in the subsequence, the discrete derivatives $\left|\partial_{x}^{\delta} h^{n}\right|$ and $\left|\partial_{y}^{\delta} h^{n}\right|$ are bounded by $C$ in $\phi(K) \cap V\left(\delta D_{n}\right)$. By a variant of the Arzela-Ascoli theorem, it then follows that there is some continuous $h^{*}: \phi(\mathbb{U}) \rightarrow[0, \infty)$ and a further subsequence such that, for every compact $K \subset \phi(\mathbb{U})$,

$$
\sup \left\{\left|h^{n}(v)-h^{*}(v)\right|: v \in K \cap \delta \mathbb{Z}^{2}\right\} \rightarrow 0
$$

along the subsequence. The same argument may also be applied to prove the convergence of the discrete derivatives of $h^{n}$ to arbitrary order, possibly in a further subsequence. Obviously, the discrete derivatives of $h^{n}$ will converge to the corresponding continuous derivatives of $h^{*}$; that is,

$$
\sup \left\{\left|\partial_{a_{1}}^{\delta} \cdots \partial_{a_{k}}^{\delta} h^{n}(v)-\partial_{a_{1}} \cdots \partial_{a_{k}} h^{*}(v)\right|: v \in K \cap \delta \mathbb{Z}^{2}\right\} \rightarrow 0,
$$

where $\partial_{a_{j}}^{\delta} \in\left\{\partial_{x}^{\delta}, \partial_{y}^{\delta}\right\}$ and $\partial_{a_{j}} \in\left\{\partial_{x}, \partial_{y}\right\}$ is the corresponding continuous derivative, $j=1,2, \ldots, k$. The fact that $h^{n}$ is discrete harmonic translates to $\left(\partial_{x}^{\delta}\right)^{2} h^{n}(v-\delta)+$ $\left(\partial_{y}^{\delta}\right)^{2} h^{n}(v-i \delta)=0$. Therefore, (5.4) shows that $h^{*}$ is harmonic. This completes the proof.

LEMMA 5.4 (Boundary hitting). For every $\epsilon_{1}, \epsilon_{2}>0$, there is a $\delta=$ $\delta\left(\epsilon_{1}, \epsilon_{2}\right)>0$ such that if $D \in \mathfrak{D}$ and $w \in V(D)$ is a vertex satisfying $\left|\psi_{D}(w)\right| \geq$ $1-\delta$, then the probability that the simple random walk started at $w$ will hit

$$
\left\{v \in V(D):\left|\psi_{D}(v)-\psi_{D}(w)\right|>\epsilon_{1}\right\}
$$

before hitting $\partial D$ is at most $\epsilon_{2}$. 
PROOF. We first prove the lemma in the case where $\epsilon_{2}$ is very close to 1 . Let $\delta>0$ be much smaller than $\epsilon_{1}$. Fix some vertex $w \in V(D)$ and suppose that $\left|\psi_{D}(w)\right| \geq 1-\delta$. Let

$$
\alpha:=\left\{z \in D:\left|\psi_{D}(z)-\psi_{D}(w)\right|=\epsilon_{1}\right\} .
$$

Let $z_{1}$ be a point in $\partial D$ closest to $w$ and set $r:=\operatorname{dist}(w, \partial D)=\left|z_{1}-w\right|$. Let $A_{1}$ be the line segment $\left[w, z_{1}\right]$. Let $Q$ be the connected component of $C\left(z_{1}, r\right) \cap D$ which contains $w$, where $C(z, r)$ denotes the circle of radius $r$ and center $z$. Then $Q$ is an arc of a circle. Let $A_{2}$ and $A_{3}$ denote the two connected components of $Q \backslash\{w\}$. See Figure 9. For $j=1,2,3$, let $K_{j}$ be the connected component of $D \backslash\left(A_{1} \cup A_{2} \cup A_{3}\right)$ which does not have $A_{j}$ as a subset of its boundary.

Because $\delta$ is small compared to $\epsilon_{1}$, the Koebe distortion theorem (e.g., Corollaries 1.4 and 1.5 in [35]) shows that $\alpha \cap C(w, r / 8)=\varnothing$. For $j=1,2,3$, let $\delta_{j}$ be the collection of all paths which stay in $K_{j}$ from the first time they hit $C(w, r / 8)$ until they first exit from $D$. Let $B(t)$ denote Brownian motion started from $w$. It is easy to see that there is a universal constant $c_{1}>0$ such that $\mathbf{P}\left[B \in s_{j}\right]>c_{1}$ for $j=1,2,3$. For example, to prove this for $j=3$, observe that the collection of Brownian paths which first hit $C(w, r / 8)$ in $K_{3}$ and later hit $A_{3}$ before $A_{1} \cup A_{2}$ has probability bounded away from 0 .

Suppose for the moment that $\alpha$ intersects $A_{1}$ and $A_{2}$. Consider a subarc $\alpha^{\prime} \subset \alpha$ whose endpoints are in $A_{1}$ and $A_{2}$, which is minimal with respect to inclusion. Then $\alpha^{\prime} \subset \overline{K_{3}}$ or $\alpha^{\prime} \subset \overline{K_{1} \cup K_{2}}$. If $\alpha^{\prime} \subset \overline{K_{3}}$, then $\alpha^{\prime}$ separates $C(w, r / 8)$ from $\partial D$ in $K_{3}$. Consequently, on the event $B \in \S_{j}, B$ hits $\alpha$ before hitting $\partial D$. However, by choosing $\delta$ to be sufficiently small and invoking the conformal invariance of the harmonic measure, we may ensure that the latter event has probability smaller than $c_{1}$. An entirely similar argument rules out the possibility that $\alpha^{\prime} \subset \overline{K_{1} \cup K_{2}}$. Similarly, it is not possible that $\alpha$ intersects both $A_{1}$ and $A_{3}$ or that $\alpha$ intersects

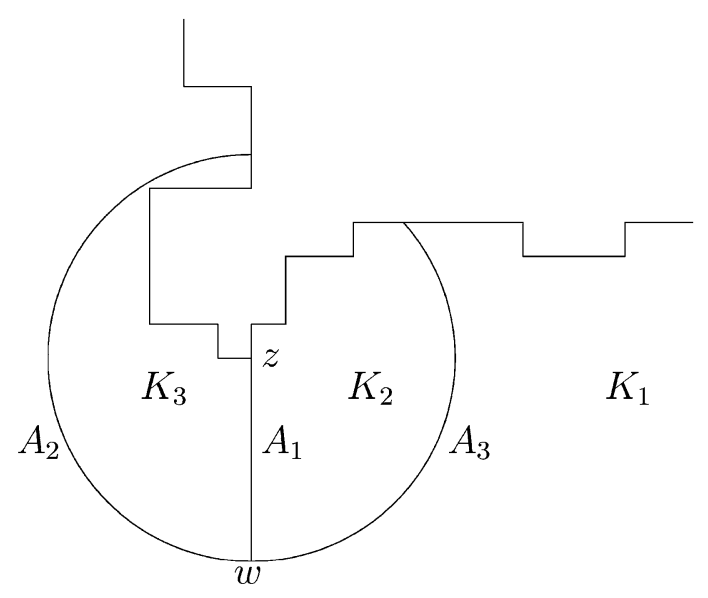

FIG. 9. The arcs $A_{j}$ and the components $K_{j}$. 
both $A_{2}$ and $A_{3}$. Hence, there is some $j \in\{1,2,3\}$ such that $\alpha \cap K_{j}=\varnothing$. Let $j^{\prime}$ be such a $j$.

By the convergence of a simple random walk to Brownian motion, it is clear that there is some universal constant $r_{0}>0$ such that if $r>r_{0}$, then the probability that the simple random walk started from $w$ is in $\delta_{j^{\prime}}$ is at least $c_{1} / 2$. This establishes the lemma in the case where $\epsilon_{2} \in\left(1-c_{1} / 2,1\right]$ and $r>r_{0}$. Suppose $r \leq r_{0}$. Then there are two grid paths of bounded length starting from $w$ to $\partial D$ that are disjoint except at $w$. If $\alpha$ intersects both these paths, then this gives a lower bound for the continuous harmonic measure of $\alpha$ from $w$. Consequently, by making $\delta$ small enough, we can make sure that this does not happen. Thus, again, with probability bounded away from 0 , the random walk from $w$ hits $\partial D$ before $\alpha$, since it may follow any one of these two paths. This proves the lemma in the case where $\epsilon_{2} \in\left(c_{2}, 1\right]$, where $c_{2}$ is some universal constant.

The Koebe distortion theorem implies that there is a constant $c>0$ such that if $v_{1}, v_{2} \in V(D)$ are neighbors, then $1-\left|\psi_{D}\left(v_{2}\right)\right| \leq c\left(1-\left|\psi_{D}\left(v_{1}\right)\right|\right)$. (See, e.g., Corollaries 1.4 and 1.5 in [35].) Consequently, we may iterate the above restricted case of the lemma and use the Markov property, thereby proving the lemma for arbitrary $\epsilon_{2}>0$.

\subsection{The hitting probability estimate.}

Proof of Proposition 2.2. Let $\epsilon_{1}>0$ be much smaller than $\epsilon$. We consider the discrete harmonic function $h(w):=H(w, u) / H(0, u)$. For $\delta>0$, let

$$
V\left(\delta, \epsilon_{1}\right):=\left\{z \in V(D):\left|\psi_{D}(z)\right| \geq 1-\delta,\left|\psi_{D}(z)-\psi_{D}(u)\right|>\epsilon_{1}\right\} .
$$

Our first goal is to show that, for every $\epsilon_{1} \in(0,1 / 4)$, there is some $\delta=\delta\left(\epsilon_{1}\right)>0$ and some $r_{0}=r_{0}\left(\epsilon_{1}\right)>0$ such that

$$
\max \left\{h(z): z \in V\left(\delta, \epsilon_{1}\right)\right\}<\epsilon_{1},
$$

provided that $\operatorname{rad}_{0}(D)>r_{0}$. This will be achieved by first showing that $h$ is not too large on the set

$$
W:=\left\{z \in V(D): \epsilon_{1} / 2 \geq\left|\psi_{D}(z)-\psi_{D}(u)\right| \geq \epsilon_{1} / 3\right\}
$$

and then letting $\delta$ go to 0 and appealing to Lemma 5.4.

Assume that $\operatorname{rad}_{0}(D)$ is sufficiently large so that any nearest neighbor path from 0 to $u$ in $D$ has a vertex in $W$. Let $M$ denote the maximum of $h$ on $W$. We claim that $M$ is bounded by a constant $c=c\left(\epsilon_{1}\right)$ depending only on $\epsilon_{1}$. Indeed, let $K$ be the set of all $v \in V(D)$ satisfying $h(v) \geq M / 2$ and let $K^{\prime}$ be the union of all edges where both endpoints are in $K \cup\{u\}$. Then the maximum principle shows that $K^{\prime}$ is connected and contains a simple nearest neighbor path $J$ joining $W$ to $u$ whose vertices are in $\left\{z \in V(D):\left|\psi_{D}(z)-\psi_{D}(u)\right| \leq \epsilon_{1} / 2\right\} \cup\{u\}$. Note that, in particular, $\operatorname{diam}\left(\psi_{D}(J)\right) \geq \epsilon_{1} / 3$. Consequently, the continuous harmonic measure from 0 of $J$ in $D$ is bounded from below by some constant $c_{1}\left(\epsilon_{1}\right)>0$. 
We claim that the discrete harmonic measure $H_{D}(0, J)$ of $J$ at the origin is also bounded away from 0 if $r_{0}$ is large enough. Indeed, let $D^{\prime}=D \backslash J$ and let $A$ be the arc on $\partial \mathbb{U}$ corresponding to $J$ under the map $\psi_{D^{\prime}}$. The length of $A$ is bounded from below, since it is equal to $2 \pi$ times the harmonic measure of $J$. Let $A^{\prime}$ denote the middle subarc of $A$ having half the length of $A$. By Lemma 5.4 applied to the domain $D^{\prime}$, it follows that there is a $c_{2}=c_{2}(\epsilon) \epsilon$ $(0,1 / 10)$, such that $H_{D^{\prime}}(v, J) \geq 1 / 2$ on vertices $v$ such that $\psi_{D^{\prime}}(v)$ is within distance $c_{2}$ of $A^{\prime}$. Using Lemma 5.3 with $\epsilon$ replaced by $c_{2} / 4$, we find that if $r_{0}$ is large, there is a nonnegative harmonic function $h_{J}^{*}: D^{\prime} \rightarrow[0, \infty)$ such that $\left|h_{J}^{*}(v)-H_{D^{\prime}}(v, J)\right| \leq c_{2} / 4$ for all $v \in V\left(D^{\prime}\right)$ satisfying $\left|\psi_{D^{\prime}}(v)\right|<1-c_{2} / 4$. Take $a \in A^{\prime}$ and $z:=\left(1-c_{2} / 2\right) a$. Then it follows from the Koebe distortion theorem (as in the argument toward the end of the proof of Proposition 3.4) that we may find a vertex $v \in V\left(D^{\prime}\right)$ such that $\left|\psi_{D^{\prime}}(v)-z\right|<c_{2} / 4$, assuming that $r_{0}$ is large enough. Thus, $h_{J}^{*}(v)>H_{D^{\prime}}(v, J)-c_{2} / 4 \geq 1 / 2-c_{2} / 4>1 / 4$. By the Harnack principle applied to $h_{J}^{*}$, there is a universal constant $c_{3}>0$ such that $h_{J}^{*}(z) \geq c_{3}$. Since this applies to every $z \in\left(1-c_{2} / 2\right) A^{\prime}$, the mean value property for $h_{J}^{*}$ gives $h_{J}^{*}(0) \geq c_{3}$ length $\left(A^{\prime}\right) /(2 \pi)$. Since $\left|h_{J}^{*}(0)-H_{D^{\prime}}(0, J)\right|<c_{2} / 4$, our claim that $H_{D}^{\prime}(0, J)$ is bounded away from 0 is established. Since $h$ is positive, harmonic $h(0)=1$ and $h \geq M / 2$ on $J$, this also gives the bound $M \leq c(\epsilon)$.

Since $h$ is harmonic, Lemma 5.4 with $\tilde{\epsilon}_{1}=\epsilon_{1} / 2$ and $\epsilon_{2}:=\tilde{\epsilon}_{1} / c(\epsilon)$ instead of $\epsilon_{1}$, $\epsilon_{2}$ implies that if $\delta=\delta\left(\epsilon_{1}\right)$ is sufficiently small, and $\operatorname{rad}_{0}(D)$ is large enough to guarantee that $W$ separates $V\left(\delta, \epsilon_{1}\right)$ from $u$ (in the graph-connectivity sense), then $h(z) \leq M \epsilon_{2}<\epsilon_{1}$ for all $z \in V\left(\delta, \epsilon_{1}\right)$ : (5.5) holds.

Now apply Lemma 5.3 again to conclude that there is a harmonic function $\hat{h}: \mathbb{U} \rightarrow[0, \infty)$ such that

$$
\left|\hat{h} \circ \psi_{D}(z)-h(z)\right|<\epsilon_{1}
$$

for all $z \in V(D)$ such that $\left|\psi_{D}(z)\right|<1-\delta / 4$. Set $\tilde{h}(z):=\hat{h}((1-\delta / 2) z)$. We know that $\tilde{h} \geq 0$ in $\partial \mathbb{U}, \tilde{h}\left(z^{\prime}\right) \leq 2 \epsilon_{1}$ on the set $S:=\left\{z^{\prime} \in \partial \mathbb{U}:\left|z^{\prime}-\psi_{D}(u)\right| \geq 2 \epsilon_{1}\right\}$. Consequently, the Poisson representation of $\tilde{h}$ gives

$$
\tilde{h}(z)=O\left(\epsilon_{1}\right)+\int_{\partial \mathbb{U} \backslash S} \tilde{h}\left(z^{\prime}\right) \frac{1-|z|^{2}}{\left|z-z^{\prime}\right|^{2}}\left|d z^{\prime}\right| .
$$

Since $\tilde{h}(0)=1+O\left(\epsilon_{1}\right)$ and $\epsilon_{1}$ is arbitrary, the proposition follows.

5.3. Some Green's function estimates. As opposed to Proposition 2.2, Lemma 3.5 requires only crude bounds. It is actually possible to prove that $G_{0}(0,0)-G_{m}(0,0)$ is close to $t_{m}$, but we do not need this result here.

Proof of Lemma 3.5. We start with (3.5). Let $S$ be the set of vertices in $V(D)$ satisfying (3.3) and assume $S \neq \varnothing$. For a random walk starting from a vertex 
in $S$, there is probability bounded away from 0 that within $\operatorname{rad}_{0}(D)^{2}$ steps it will exit $D$. This gives

$$
\sum_{w \in S} G_{D}(0, w) \leq O(1) \operatorname{rad}_{0}(D)^{2}
$$

On the other hand, with probability bounded away from 0 , the number of steps into vertices in $S$ for the random walk started at 0 that is stopped on exiting $D$ is greater than $\operatorname{rad}_{0}(D)^{2}$. Therefore,

$$
O(1) \sum_{w \in S} G_{D}(0, w) \geq \operatorname{rad}_{0}(D)^{2} .
$$

By reversing the walk, we know that $G_{D}(0, w)=G_{D}(w, 0)$. Since $G_{D}(w, 0)$ is harmonic on $V(D) \backslash\{0\}$, the Harnack principle [i.e., $k=0$ in (5.2)] can be used to show that $G_{D}(w, 0) / G_{D}\left(w^{\prime}, 0\right)=O(1)$ when $w, w^{\prime} \in S$. Combining this with $G_{D}(0, w)=G_{D}(w, 0)$ and the estimates (5.6), (5.7) gives (3.5).

By Lemma 2.1, we have

$$
\operatorname{diam}\left(\psi_{D} \circ \gamma[0, m]\right)=O(\delta) .
$$

In the following, we fix $\gamma[0, m]$ (i.e., it will be considered deterministic). Let $z$ be the vertex where a simple random walk from 0 first exits $D_{m}$. By considering what happens to the random walk after first hitting $z$, we get the identity $G_{0}(0, v)-G_{m}(0, v)=\mathbf{E}\left[G_{0}(z, v)\right]$ [where $G_{0}(z, v)=0$ for $z \notin V(D)$, by definition]. Consequently,

$$
G_{0}(0, v)-G_{m}(0, v) \leq \mathbf{P}[z \in \gamma[0, m]] \max \left\{\mathbf{E}\left[G_{0}\left(\gamma_{j}, v\right)\right]: j=1, \ldots, m\right\} .
$$

By (5.8), the continuous harmonic measure from 0 of $\psi_{D} \circ \gamma[0, m]$ in $\mathbb{U}$ is $O(\delta)$. Therefore, the continuous harmonic measure from 0 of $\gamma[0, m]$ in $D$ is also $O(\delta)$. As in the argument given in Section 5.2, this implies that if $\operatorname{rad}_{0}(D)$ is large enough, $\mathbf{P}[z \in \gamma[0, m]]=O(\delta)$.

Let $K$ denote the disk $\left\{w \in D:|w-v|<\operatorname{rad}_{0}(D) / 10\right\}$ and fix some $j \in\{1$, $2, \ldots, m\}$. Since $\psi_{D} \circ \gamma[0, m]$ is contained in $\overline{\mathbb{U}} \backslash(1-O(\delta)) \mathbb{U}$. It follows that the continuous harmonic measure of $K$ from $\gamma_{j}$ in $D$ is $O(\delta)$. If $\psi_{D}\left(\gamma_{j}\right)$ is sufficiently close to $\partial \mathbb{U}$ (how close may depend on $\delta$ ), then we can make sure that the corresponding discrete harmonic measure $H_{D}\left(\gamma_{j}, K\right)$ is less than $\delta$ by Lemma 5.4. If $\psi_{D}\left(\gamma_{j}\right)$ is not close to $\partial \mathbb{U}$, then when $\operatorname{rad}_{0}(D)$ is large the bound $H_{D}\left(\gamma_{j}, K\right) \leq O(\delta)$ follows by the convergence of the discrete harmonic measure to the continuous harmonic measure, as we have seen before. If $w \in V(D) \cap K$ neighbors with a vertex outside of $K$, then $G_{0}(w, v)=O(1)$ follows from (5.7) by translating $w$ to 0 . Hence, $G_{0}\left(\gamma_{j}, v\right)=O(1) H_{D}\left(\gamma_{j}, K\right)=O(\delta)$. Putting these estimates together completes the proof. 
5.4. Mixed boundary conditions. Recall that Proposition 4.2, which we will now prove, is not used in the proof of Theorem 1.1.

Proof of Proposition 4.2. Suppose first that the distance between $A_{0}$ and $A_{1}$ is at least $\epsilon$. Let $A_{0}^{*}$ and $A_{1}^{*}$ denote the two connected components of $\eta \backslash\left(A_{0}^{\prime} \cup A_{1}^{\prime}\right)$ such that the sequence $\left(A_{0}^{\prime}, A_{0}^{*}, A_{1}^{\prime}, A_{1}^{*}\right)$ conforms to the counterclockwise order along $\eta$. This induces a corresponding partition $E_{2}=$ $E_{0}^{*} \cup E_{1}^{*}$ of $E_{2}$, according to whether or not the first point on the edge is in $A_{0}^{*}$ or in $A_{1}^{*}$.

We need to use the discrete harmonic conjugate function $\hat{k}$ of $\hat{h}$. To be perfectly precise, it is necessary to set some combinatorial infrastructure: we first define a (multi-) graph $\hat{H}$, and $\hat{k}$ will be defined on the planar dual $\hat{H}^{\dagger}$ of $\hat{H}$. The vertices of $\hat{H}$ are $V_{H} \cup\left\{v_{0}, v_{1}\right\}$ (where $v_{0}$ and $v_{1}$ are new symbols not appearing in $V_{H}$ ). As edges of $\hat{H}$, we take all the edges of $H$, and, additionally, for every $j=0,1$ and every directed edge $[v, u]$ in $E_{j}$, there is a corresponding edge $\left[v, v_{j}\right]$ in $\hat{H}$. Finally, there is also the edge $\left[v_{0}, v_{1}\right]$ in $\hat{H}$. Consider a planar embedding of $\hat{H}$ which extends the planar embedding of $H$ such that $v_{0}$ and $v_{1}$ are in the unbounded component of $\mathbb{C} \backslash H$. Let $\hat{H}^{\dagger}$ denote the planar dual of $\hat{H}$. Then there is a unique edge $\left[v_{0}^{\dagger}, v_{1}^{\dagger}\right]$ in $\hat{H}^{\dagger}$ which crosses $\left[v_{0}, v_{1}\right]$. We choose the labels so that $v_{j}^{\dagger}$ naturally corresponds to $A_{j}^{*}, j=0,1$. Set $\hat{h}\left(v_{j}\right):=j, j=0,1$. If we consider $\hat{h}$ as a function on $\hat{H}$, then it is discrete harmonic except at $v_{0}$ and $v_{1}$. This easily implies (see, e.g., [7] or, more explicitly, [4]) that there is a discrete harmonic conjugate $\hat{k}$ defined on the vertices of $\hat{H}^{\dagger}$; that is, for every directed edge $e=[u, v]$ in $\hat{H}$ if $\{u, v\} \neq\left\{v_{0}, v_{1}\right\}$, then the discrete Cauchy-Riemann equation $\hat{h}(v)-\hat{h}(u)=\hat{k}\left(v^{\dagger}\right)-\hat{k}\left(u^{\dagger}\right)$ holds, where $\left[u^{\dagger}, v^{\dagger}\right]$ is the edge of $\hat{H}^{\dagger}$ intersecting $e$ from right to left. In fact, $\hat{k}$ is harmonic in $\hat{H}^{\dagger}$ except at $v_{0}^{\dagger}$ and $v_{1}^{\dagger}$. The function $\hat{k}$ is unique, up to an additive constant. We choose the additive constant so that $\hat{k}\left(v_{0}^{\dagger}\right)=0$. Since $\hat{h} \geq 0$, by considering the neighbors of $v_{0}$ and the orientation, it follows that $\hat{k}\left(v_{1}^{\dagger}\right) \geq 0$.

Consider a sequence $D_{n}$ of such domains satisfying $\operatorname{rad}_{0}\left(D_{n}\right) \geq n$, with $\operatorname{arcs}$ $\eta=\eta_{n}$ and such harmonic functions $\hat{h}_{n}, \hat{k}_{n}$. Let $L_{n}$ denote the maximum value of $\hat{k}_{n}$, which is the value of $\hat{k}_{n}$ on $v_{1}^{\dagger}$.

Since $\epsilon>0$ is fixed, we can consider a subsequence of $n \rightarrow \infty$ such that the arcs $A_{0}$ and $A_{1}$ converge to $\operatorname{arcs} \tilde{A}_{0}$ and $\tilde{A}_{1}$ of length at least $\epsilon$, and the distance between them is at least $\epsilon$. Let $\tilde{A}_{0}^{*}$ and $\tilde{A}_{1}^{*}$ denote the two components of $\partial \mathbb{U} \backslash\left(\tilde{A}_{0} \cup \tilde{A}_{1}\right)$, so that $\tilde{A}_{0}, \tilde{A}_{0}^{*}, \tilde{A}_{1}, \tilde{A}_{1}^{*}$ is the positive order along $\partial \mathbb{U}$ of these arcs.

We now separate the argument into two cases according to whether or not $L_{n}>1$. Suppose that $L_{n}>1$ for infinitely many $n$ and take a further subsequence of $n$ such that $L_{n}>1$ along that subsequence. Then $\hat{k}_{n} / L_{n}$ and $\hat{h}_{n} / L_{n}$ are both bounded by 1 . It follows from Lemma 5.3 that after taking a further subsequence, if necessary, there are harmonic functions $h$ and $k$ on $\mathbb{U}$ 
such that $L_{n}^{-1} \hat{h}_{n} \circ \psi_{D_{n}}^{-1} \rightarrow h$ and $L_{n}^{-1} \hat{k}_{n} \circ \psi_{D_{n}}^{-1} \rightarrow k$ uniformly on compact subsets of $\mathbb{U}$ (appropriately interpreted, since $\hat{h}_{n}$ and $\hat{k}_{n}$ are only defined on vertices and dual vertices, not on every point of $D_{n}$ ). Moreover, (5.4) shows that $h$ and $k$ are harmonic conjugates, because the discrete Cauchy-Riemann equations tend to the continuous Cauchy-Riemann equations.

By Lemma 5.4, it follows that $k$ is, respectively, equal to 0 and 1 in the relative interior of $\tilde{A}_{0}^{*}, \tilde{A}_{1}^{*}$, and similarly $h$ has boundary values 0 and $1 / \tilde{L}$ in $\tilde{A}_{0}$ and $\tilde{A}_{1}$, where $\tilde{L}:=\lim _{n \rightarrow \infty} L_{n}$ (where the limit is along the subsequence and must exist and be finite). By Schwarz reflection, say, this implies that $h$ and $k$ satisfy Neumann boundary conditions in $\tilde{A}_{0}^{*} \cup \tilde{A}_{1}^{*}$ and $\tilde{A}_{0} \cup \tilde{A}_{1}$, respectively. It now easily follows (e.g., from the maximum principle) that $h+i k$ is the (unique) conformal map taking $\mathbb{U}$ to the rectangle $[0,1 / \tilde{L}] \times[0,1]$ which takes the four arcs $\tilde{A}_{0}, \tilde{A}_{0}^{*}, \tilde{A}_{1}, \tilde{A}_{1}^{*}$ to the corresponding sides of the rectangle.

The argument in the case where $L_{n} \leq 1$ for infinitely many $n$ proceeds in the same manner, except that one should not divide $\hat{h}_{n} \circ \psi_{D_{n}}^{-1}$ and $\hat{k}_{n} \circ \psi_{D_{n}}^{-1}$ by $L_{n}$.

It remains to remove the assumption that the distance between $A_{0}$ and $A_{1}$ is at least $\epsilon$. Observe that the probabilistic description of $\hat{h}$ shows that it is monotone increasing in $A_{1}^{\prime}$ and monotone decreasing in $A_{0}^{\prime}$. Take $\epsilon^{\prime}>0$ much smaller than $\epsilon$. Then $\hat{h}(0)$ for the given configuration is bounded from above by the value of $\hat{h}(0)$ for the configuration where arcs of length $\epsilon^{\prime}$ are removed at the two ends of $A_{1}$, and $A_{1}^{\prime}$ is adjusted accordingly. Similarly, $\hat{h}(0)$ is bounded from below by the value of $\hat{h}(0)$ for the configuration where such arcs are removed at the two ends of $A_{0}$. The difference between the value of $h$ for the original versus any of the modified configurations goes to 0 as $\epsilon^{\prime} \rightarrow 0$, since $h$ depends continuously on ( $A_{1}, A_{2}$ ), as long as the length of $A_{1} \cup A_{2}$ is not 0 . Consequently, we get the proposition by applying the restricted version proved above with $\epsilon^{\prime}$ in place of $\epsilon$ and by "sandwiching."

6. Other lattices. For convenience and simplicity, the proofs up to now have been written for the loop-erased random walk and UST Peano curve on the square grid. The purpose of this section is to briefly indicate how to adapt the proofs to more general walks on more general grids. In order to keep this section short, we will not try to consider the most general cases.

Let $L$ be a (strictly two-dimensional) lattice in $\mathbb{R}^{2}$; that is, $L$ is a discrete additive subgroup of $\mathbb{R}^{2}$ that is not contained in a line. Discrete means that there is some neighborhood of 0 whose intersection with $L$ is $\{0\}$. Suppose that $G$ is a planar graph whose vertices are the elements of $L$, and $G$ is invariant under translation by elements of $L$. That is, if $u, v \in L$ are neighbors in $G$ and $\ell \in L$, then $\ell+u$ neighbors $\ell+v$. It is not hard to verify that there is a linear map taking $L$ to the triangular lattice such that neighbors in $G$ are mapped to vertices at distance 1 . In particular, as a graph, $G$ is isomorphic to the triangular grid or to the square grid. 
Let $N$ be the set of neighbors of 0 in $G$ and let $N^{\prime}:=\{0\} \cup N$. Let $X$ be an $N^{\prime}$-valued random variable and let $X_{1}, X_{2}, \ldots$ be an i.i.d. sequence where each $X_{n}$ has the same law as $X$. Consider the random walk

$$
S_{n}:=\sum_{j=1}^{n} X_{j}
$$

on $G$. We are interested in the situation where the scaling limit of $S_{n}$ is standard Brownian motion. For this purpose, we require that $\mathbf{E}[X]=0$ and that the covariance matrix of $X$ is the identity matrix. (Note that if the covariance matrix of $X$ is nondegenerate but not equal to the identity, we can always apply a linear transformation to the system to convert to the above situation. Therefore, what we say below also applies in that case, provided that we appropriately modify the linear complex structure on $\mathbb{R}^{2}$.)

Note that, under these assumptions, the Markov chain corresponding to the walk $S_{n}$ does not need to be reversible. An interesting particular example the reader may wish to keep in mind is where $\mathbf{P}[X=\exp (2 \pi i j / 3)]=1 / 3$ for $j=0,1,2$.

THEOREM 6.1. Theorem 1.1 applies to the loop erasure of the random walk $S_{n}$.

PROOF. An inspection of the proof of Theorem 1.1, including all the necessary lemmas, shows that only the generalization of the proof of Lemma 5.1 to the present framework requires special justification, which is given below.

LEMMA 6.2. Let $\tau_{r}$ denote the first time $n$ with $\left|S_{n}\right| \geq r$. There exists a constant $C$, depending on $X$ but not on $r$, such that, for all $r \geq C, w \in N$ and $y \in L$,

$$
\left|\mathbf{P}^{0}\left[S_{\tau_{r}}=y\right]-\mathbf{P}^{w}\left[S_{\tau_{r}}=y\right]\right| \leq C r^{-1} \mathbf{P}^{0}\left[S_{\tau_{r}}=y\right] .
$$

Here, $\mathbf{P}^{w}$ denotes the law of the Markov chain started from $w$; that is, the law of $\left(S_{n}+w: n \in \mathbb{N}\right)$ under $\mathbf{P}=\mathbf{P}^{0}$. This lemma is clearly sufficient to provide the necessary analog of Lemma 5.1 for $S_{n}$.

ProOF OF LEMMA 6.2. There are various ways to prove the lemma (via coupling, for instance). We give here a proof based on the Green's functions, as in [25]. Without loss of generality, we assume that $\mathbf{P}[X=0]>0$ and that $L$ is the minimal lattice containing $\left\{w \in N: p_{w}>0\right\}$. Then the random walk is irreducible on $L$. The discrete Laplacian $\Delta_{X}$ associated with $X$ is defined by

$$
\Delta_{X} f(z):=\mathbf{E}[f(z+X)]-f(z) .
$$

Let $a$ be the potential kernel for the random walk,

$$
a(z):=\sum_{j=0}^{\infty}\left(\mathbf{P}^{0}\left[S_{j}=0\right]-\mathbf{P}^{0}\left[S_{j}=-z\right]\right) .
$$


It is known that the series converges and, in fact,

$$
a(z)=c_{1} \log |z|+c_{2}+O\left(|z|^{-1}\right)
$$

as $|z| \rightarrow \infty, z \in L$ (where $c_{1}, c_{2}$ depend on the law of $X$ ). This is proved in [12] for the lattice $\mathbb{Z}^{2}$ with arbitrary nondegenerate covariance matrix (with an appropriate dependence on the matrix), so the above follows for other $L$ by applying a linear transformation. Since $\mathbf{P}^{0}\left[S_{j}=-z\right]=\mathbf{P}^{z}\left[S_{j}=0\right]$, it follows that

$$
\Delta_{X} a(z)= \begin{cases}1, & z=0 \\ 0, & z \neq 0 .\end{cases}
$$

Let $G_{r}$ denote the Green's function for the walk in $L \cap r \mathbb{U}$, that is, $G_{r}\left(z, z^{\prime}\right):=$ $\sum_{j \in \mathbb{N}} \mathbf{P}^{z}\left[j<\tau_{r}, S_{j}=z^{\prime}\right]$. Note that, for all $z, w \in L \cap r \mathbb{U}$,

$$
a(z-w)+G_{r}(z, w)=\mathbf{E}^{z}\left[a\left(S_{\tau_{r}}-w\right)\right],
$$

since for fixed $w$ both sides are $\Delta_{X}$-harmonic for $z \in L \cap r \mathbb{U}$ and equality holds for $z \in L \backslash r \mathbb{U}$. Set $M:=\max \{|w|: w \in N\}$ and $Z=\{z \in L: r / 2 \leq|z|<M+r / 2\}$. By (6.1) and (6.2), $G_{r}(z, w)=c_{1} \log 2+O\left(r^{-1}\right)$ for $z \in Z$ and $w \in N^{\prime}$. The same argument applied to the reverse walk $-S_{j}$, which has potential kernel $\bar{a}(z)=a(-z)$ and Green's function $\bar{G}_{r}(z, w)=G_{r}(w, z)$, gives

$$
\forall w \in N^{\prime}, \forall z \in Z, \quad G_{r}(w, z)=c_{1} \log 2+O\left(r^{-1}\right) .
$$

Assuming $r>4 M$, by considering the last vertex in $Z$ visited by the walk before time $\tau_{r}$, we obtain, for all $w \in N^{\prime}$ and all $y \in L$,

$$
\mathbf{P}^{w}\left[S_{\tau_{r}}=y\right]=\sum_{z \in Z} G_{r}(w, z) \mathbf{P}^{z}\left[S_{\tau_{r}}=y, \min \left\{j \geq 1: S_{j} \in Z\right\}>\tau_{r}\right] .
$$

Together with (6.3), this completes the proof of the lemma.

Observe that Theorem 1.1 also holds for the simple random walk on the honeycomb grid, because two steps on the honeycomb lattice are the same as a single step on a triangular grid containing every other vertex on the honeycomb grid, and so Lemma 6.2 may be applied.

We now turn our attention to spanning trees and the generalizations of Corollary 1.2 and Theorem 1.3. Suppose that $X$ and $-X$ have the same distribution, so that the walk $S$ is reversible. For an edge $e=[x, y]$, define $p_{e}=\mathbf{P}[X=y-x]=\mathbf{P}[X=x-y]$. In this case, it is easy to generalize the definition of UST to a measure on trees related to the law of $X$. This can be done either by using Wilson's algorithm or, equivalently, by giving to each tree $T$ a probability that is proportional to the product of the transition probabilities along the edges of $T$. In other words, $\mathbf{P}[T]=Z^{-1} \prod_{e \in T} p_{e}$, where $Z$ is a normalizing constant. (The equivalence is proved in [44]; see also [26].) We call this the UST corresponding to the walk $S$ (even if this probability measure is not uniform). Note that Lemma 4.1 also holds in the present setting because the probability $\mathbf{P}[T]$ is given in terms of a product. 
THEOREM 6.3. Assuming that $-X$ has the same distribution as $X$ (i.e., $S_{j}$ is reversible), Corollary 1.2 and Theorem 1.3 hold for the UST corresponding to the walk $S$.

PROOF. The proof of Corollary 1.2 holds in this generality. In the proof of Theorem 1.3, the only significant changes concern the discrete harmonic conjugate function, used in the proof of Proposition 4.2. Recall that there is an appropriate definition for the discrete harmonic conjugate for reversible walks on planar graphs, where the discrete Cauchy-Riemann equation is modified (see [7] or [19], Section 6.1). If $G$ is graph isomorphic to the square grid, the same is true for the dual graph. If $G$ is graph isomorphic to the triangular grid, then the dual is graph isomorphic to the honeycomb grid. As pointed out above, Lemma 6.2 may therefore be applied to the harmonic conjugate. The details are left to the reader.

In the nonreversible setting, instead of a spanning tree, one should consider a spanning arborescence, which is an oriented tree with a root and the edges are oriented toward the root. Fix a finite Markov chain with state space $V$ and a root $o \in V$. Consider the measure on spanning arborescences of $V$ with root $o$, where the probability for $T$ is proportional to the product of the transition probabilities along the directed edges of $T$. This is the analog of the UST in the nonreversible setting. Wilson's algorithm holds in this generality (see [44]); however, the choice of the root $o$ clearly matters.

If we consider a finite piece of the lattice $L$, and we wire part or all of the boundary, it is natural to pick the wired vertex as the root. With this convention, Corollary 1.2 holds for the wired tree. It would be interesting to see if the free tree with root chosen at $0 \in D$ is invariant under conformal maps preserving 0 , say (in the nonreversible setting). Of course, one needs to choose a grid approximation of $D$ where there is an oriented path from each vertex to the root 0 .

In the proof of Theorem 1.3, we have used reversibility in two places. The proofs of Theorems 10.7 and 11.1 of [38], which we quoted, currently require reversibility. However, these results were only used to improve the topology of convergence to SLE. More seriously, Section 5.4 uses the conjugate harmonic function, whose definition in the nonreversible setting is not clear. Notwithstanding the obstacles, it seems likely that these results can be proven in the nonreversible setting, too.

\section{REFERENCES}

[1] Ahlfors, L. V. (1973). Conformal Invariants: Topics in Geometric Function Theory. McGraw-Hill, New York.

[2] Aizenman, M. and Burchard, A. (1999). Hölder regularity and dimension bounds for random curves. Duke Math. J. 99 419-453. 
[3] Aizenman, M., Burchard, A., Newman, C. M. and Wilson, D. B. (1997). Scaling limits for minimal and random spanning trees in two dimensions. Random Structures Algorithms 15 319-367.

[4] Benjamini, I. and Schramm, O. (1996). Random walks and harmonic functions on infinite planar graphs using square tilings. Ann. Probab. 24 1219-1238.

[5] Cardy, J. L. (1992). Critical percolation in finite geometries. J. Phys. A 25 L201-L206.

[6] Collatz, L. (1960). The Numerical Treatment of Differential Equations, 3rd ed. Springer, Berlin.

[7] DeHn, M. (1903). Über die Zerlegung von Rechtecken in Rechtecke. Math. Ann. 57 314-332.

[8] Dubins, L. E. (1968). On a theorem of Skorohod. Ann. Math. Statist. 39 2094-2097.

[9] Dudley, R. M. (1989). Real Analysis and Probability. Wadsworth and Brooks/Cole, Pacific Grove, CA.

[10] Duplantier, B. (1987). Critical exponents of Manhattan Hamiltonian walks in two dimensions, from Potts and $o(n)$ models. J. Statist. Phys. 49 411-431.

[11] DuPlantier, B. (1992). Loop-erased self-avoiding walks in two dimensions: Exact critical exponents and winding numbers. Phys. A 191 516-522.

[12] Fomin, S. (2001). Loop-erased walks and total positivity. Trans. Amer. Math. Soc. 353 3563-3583.

[13] Fukai, Y. and UchiYama, K. (1996). Potential kernel for two-dimensional random walk. Ann. Probab. 24 1979-1992.

[14] Guttmann, A. and Bursill, R. (1990). Critical exponent for the loop-erased self-avoiding walk by Monte-Carlo methods. J. Statist. Phys. 59 1-9.

[15] HÄGgStröm, O. (1995). Random-cluster measures and uniform spanning trees. Stochastic Process. Appl. 59 267-275.

[16] He, Z.-X. and Schramm, O. (1998). The $C^{\infty}$-convergence of hexagonal disk packings to the Riemann map. Acta Math. 180 219-245.

[17] Karatzas, I. and Shreve, S. E. (1988). Brownian Motion and Stochastic Calculus. Springer, New York.

[18] Kasteleyn, P. W. (1963). A soluble self-avoiding walk problem. Physica 29 1329-1337.

[19] KenYon, R. (1998). Tilings and discrete Dirichlet problems. Israel J. Math. 105 61-84.

[20] Kenyon, R. (2000). The asymptotic determinant of the discrete Laplacian. Acta Math. 185 239-286.

[21] Kenyon, R. (2000). Long-range properties of spanning trees. J. Math. Phys. 41 1338-1363.

[22] Kenyon, R. (2000). Conformal invariance of domino tilings. Ann. Probab. 28 759-795.

[23] KozmA, G. (2002). Scaling limit of loop-erased random walks: A naive approach. Unpublished manuscript.

[24] Lawler, G. F. (1980). A self-avoiding random walk. Duke Math. J. 47 655-693.

[25] LAWLER, G. F. (1991). Intersections of Random Walks. Birkhäuser, Boston.

[26] LaWler, G. F. (1999). Loop-erased random walk. In Perplexing Problems in Probability 197-217. Birkhäuser, Boston.

[27] Lawler, G. F., Schramm, O. and Werner, W. (2001). Values of Brownian intersection exponents I. Half-plane exponents. Acta Math. 187 237-273.

[28] Lawler, G. F., Schramm, O. and Werner, W. (2001). Values of Brownian intersection exponents II. Plane exponents. Acta Math. 187 275-308.

[29] LaWler, G. F., Schramm, O. and Werner, W. (2002). Analyticity of intersection exponents for planar Brownian motion. Acta Math. 189 179-201.

[30] LaWler, G. F., Schramm, O. and Werner, W. (2002). One-arm exponent for critical 2D percolation. Electron. J. Probab. 7 No. 2.

[31] Lawler, G. F., Schramm, O. and Werner, W. (2003). Conformal restriction properties: The chordal case. J. Amer. Math. Soc. 16 917-955. 
[32] LyONS, R. (1998). A bird's-eye view of uniform spanning trees and forests. In Microsurveys in Discrete Probability 135-162. Amer. Math. Soc., Providence, RI.

[33] Majumdar, S. N. (1992). Exact fractal dimension of the loop-erased self-avoiding walk in two dimensions. Phys. Rev. Lett. 68 2329-2331.

[34] Pemantle, R. (1991). Choosing a spanning tree for the integer lattice uniformly. Ann. Probab. 19 1559-1574.

[35] Pommerenke, Ch. (1992). Boundary Behaviour of Conformal Maps. Springer, Berlin.

[36] Revuz, D. and Yor, M. (1991). Continuous Martingales and Brownian Motion. Springer, Berlin.

[37] Rohde, S. and Schramm, O. (2001). Basic properties of SLE. Unpublished manuscript.

[38] SCHR AMM, O. (2000). Scaling limits of loop-erased random walks and uniform spanning trees. Israel J. Math. 118 221-288.

[39] Schramm, O. (2001). A percolation formula. Electron. Comm. Probab. 6 115-120.

[40] SMIRnOv, S. (2001). Critical percolation in the plane: Conformal invariance, Cardy's formula, scaling limits. C. R. Acad. Sci. Paris Sér. I Math. 333 239-244.

[41] SMirnov, S. (2001). Critical percolation in the plane. I. Conformal invariance and Cardy's formula. II. Continuum scaling limit. Preprint.

[42] Smirnov, S. and Werner, W. (2001). Critical exponents for two-dimensional percolation. Math. Res. Lett. 8 729-744.

[43] Strassen, V. (1967). Almost sure behavior of sums of independent random variables and martingales. Proc. Fifth Berkeley Symp. Math. Statist. Probab. 315-343. Univ. California Press.

[44] WILSON, D. B. (1996). Generating random spanning trees more quickly than the cover time. In Proceedings of the 28th Annual ACM Symposium on the Theory of Computing 296-303. ACM, New York.

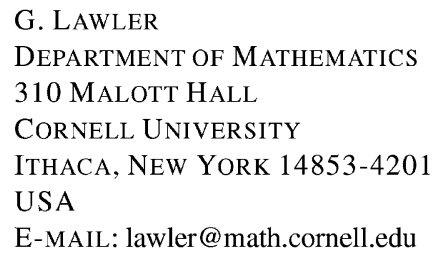

E-MAIL: lawler@math.cornell.edu

\author{
O. SCHRAMM \\ MICROSOFT CORPORATION \\ ONE MICROSOFT WAY \\ REDMOND, WASHINGTON 98052 \\ USA \\ E-MAIL: schramm@microsoft.com
}

\author{
W. WERNER \\ DÉPARTEMENT DE MATHÉMATIQUES \\ BÂT. 425 \\ UNIVERSITÉ PARIS-SUD \\ 91405 ORSAY CEDEX \\ FRANCE \\ E-MAIL: wendelin.werner@math.u-psud.fr
}

